\title{
Diapycnal Advection by Double Diffusion and Turbulence in the Ocean
}

\author{
by \\ Louis Christopher St. Laurent \\ B.S. University of Rhode Island, 1994 \\ Submitted in partial fulfillment of the requirements for the degree of \\ Doctor of Philosophy \\ at the \\ MASSACHUSETTS INSTITUTE OF TECHNOLOGY \\ and the \\ WOODS HOLE OCEANOGRAPHIC INSTITUTION \\ September 1999 \\ (c)1999 Louis C. St. Laurent. All rights reserved.
}

The author hereby grants to MIT and to WHOI permission to reproduce and to distribute copies of this thesis document in whole or in part.

Signature of Author

Joint Hrogram in Physical Oceanography

Massachusetts Institute of Technology

Woods Hole Oceanographic Institute

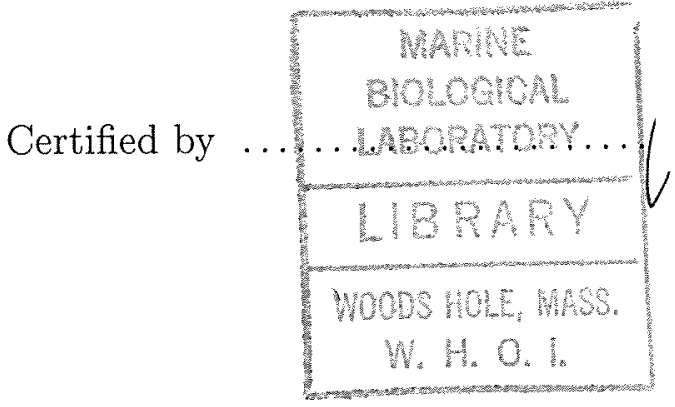

Accepted by $\ldots \ldots \ldots \ldots \ldots \ldots$
August 4, 1999

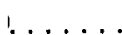

V

Raymond W. Schmitt

Senior Scientist

Woods Hole Oceanographic Institute

Thesis Supervisor

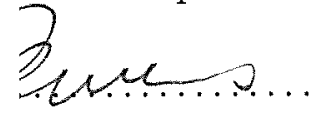

W. Brechner Owens

Chairman, Joint Committee for Physical Oceanography Massachusetts Institute of Technology Woods Hole Oceanographic Institute 


\title{
Diapycnal Advection by Double Diffusion and Turbulence in the Ocean
}

by

\author{
Louis C. St. Laurent
}

Submitted in partial fulfillment of the requirements for the degree of Doctor of Philosophy at the Massachusetts Institute of Technology and the Woods Hole

Oceanographic Institution in August 1999

\begin{abstract}
Observations of diapycnal mixing rates are examined and related to diapycnal advection for both double-diffusive and turbulent regimes.

The role of double-diffusive mixing at the site of the North Atlantic Tracer Release Experiment is considered. The strength of salt-finger mixing is analyzed in terms of the stability parameters for shear and double-diffusive convection, and a nondimensional ratio of the thermal and energy dissipation rates. While the model for turbulence describes most dissipation occurring in high shear, dissipation in low shear is better described by the salt-finger model, and a method for estimating the salt-finger enhancement of the diapycnal haline diffusivity over the thermal diffusivity is proposed. Best agreement between tracer-inferred mixing rates and microstructure based estimates is achieved when the salt-finger enhancement of haline flux is taken into account.

The role of turbulence occurring above rough bathymetry in the abyssal Brazil Basin is also considered. The mixing levels along sloping bathymetry exceed the levels observed on ridge crests and canyon floors. Additionally, mixing levels modulate in phase with the spring-neap tidal cycle. A model of the dissipation rate is derived and used to specify the turbulent mixing rate and constrain the diapycnal advection in an inverse model for the steady circulation. The inverse model solution reveals the presence of a secondary circulation with zonal character. These results suggest that mixing in abyssal canyons plays an important role in the mass budget of Antarctic Bottom Water.

Thesis Supervisor: Raymond W. Schmitt

Title: Senior Scientist, Woods Hole Oceanographic Institution
\end{abstract}




\section{Acknowledgments}

In my final year as an undergraduate at the University of Rhode Island, I spoke with an adjunct faculty member of the physics department about my interests in physical oceanography. Lou Goodman immediately mentioned the MIT/WHOI program. In particular, he wrote the names of several faculty members on a piece of paper, adding, "I respect these people not only as scientists, but also as friends." A day later, I found brief descriptions of research interests for each of the names Lou provided, and I decided to make some calls. Ray Schmitt was first name on the list. His research sounded interesting, and besides, he was very friendly on the phone. I didn't see any need to call the remaining people.

My initial judgment was appropriate. Ray's enthusiasm is contagious, and he introduced me to numerous exciting problems in oceanography. The work I began with Ray my first summer became the seed for my thesis work. His guidance allowed me to make steady progress on interesting problems while I was still learning the ropes. I am fortunate to have found such a competent mentor.

As a member of a research team, my thesis work would not have progressed without the additional guidance of others. The scope of John Toole's expertise is rivaled by few in physical oceanography. He has tackled problems ranging from the mass budget of the Indian Ocean (a spatial scale of millions of meters) to the molecular dissipation of scalar variance (a spatial scale of millimeters). I have exploited many of John's insights in my work. Kurt Polzin too has been a great asset. The quality of the microstructure data on which my work relied was assured by Kurt's efforts. Francesco Paparella joined the team just in time to allow me to run memory-greedy calculations on his workstation. Finally, the group's technical support, in particular Ellyn Montgomery and Gwyneth Packard, maintained the resources I used everyday.

My experience in graduate school was overwhelmingly positive, largely because of the people I met. My close classmates, Brian Arbic, Albert Fischer, Stephanie Harrington and Steve Jayne deserve special mention. Tom Marchitto, Mike Braun and Patricia Kassis also became close friends.

There are only a few people who know the whole story, which is probably for the best. My friends often hear me joke about how I was not a member of the 
"gifted class." The joke would not be lost on Jack Crowley. Most of all, I have shared so much of my story with a geologist I met at URI. When family friends learn that my research doesn't involve dolphins, it is Jenn who saves the moment for science, with stories of her volcano adventures. There are many other things that Jenn does so well, and it is to her that I am the most grateful.

This work was supported by contracts N00014-92-1323 and N00014-97-10087 of the Office of Naval Research and grant OCE94-15589 of the National Science Foundation. 


\section{Contents}

1 Introduction $\quad 7$

1.1 Thermodynamic Considerations . . . . . . . . . . . . . . . 12

1.2 Dynamical Considerations . . . . . . . . . . . . 15

2 The Contribution of Salt Fingers to Vertical Mixing in the North Atlantic Tracer Release Experiment $\quad 19$

2.1 Introduction . . . . . . . . . . . . . . . . 19

2.2 Qualitative Evidence for Salt Fingers at the NATRE Site . . . . 22

2.3 Quantitative Assessment of Salt-Finger Mixing . . . . . . . . 24

2.3.1 Description of Data . . . . . . . . . . . . 24

2.3.2 The Dissipation Ratio Model . . . . . . . . . . . . . . 28

2.3.3 Method of Analysis . . . . . . . . . . . . . . 31

2.3.4 Statistical Treatment of Dissipation Data . . . . . . . 35

2.3.5 Results ................... 38

2.4 Mixing at the NATRE Site . . . . . . . . . . . . . . 46

2.4.1 Vertical Mixing Influenced by Salt Fingers . . . . . . . . . 46

2.4.2 Estimates of Diapycnal Advection . . . . . . . . . 51

2.5 Discussion .................... 56

3 Buoyancy Forcing Above Rough Bathymetry in the Abyssal Brazil Basin 63

3.1 Introduction . . . . . . . . . . . . . . . . 63

3.2 Dissipation ..................... 67

3.2.1 Description of Dissipation Data ......... . 67 
Spatial Bias . . . . . . . . . . . . . . . 73

Temporal Bias . . . . . . . . . . . . . . . . . 73

3.2 .2 Mechanisms of Bottom Generation . . . . . . . . 76

3.2 .3 A Model for the Dissipation . . . . . . . . . . . . . . 79

3.3 The Inverse Model . . . . . . . . . . . . . . . . . . . 80

3.3.1 Model Equations . . . . . . . . . . . . . . . . . . . 80

3.3.2 Treatment of Data . . . . . . . . . . . . . . . . 82

3.3.3 Linear Algebraic Equations . . . . . . . . . . . . . . 88

3.3 .4 Results . . . . . . . . . . . . . . . . . . . 89

Shallow Flow . . . . . . . . . . . . . . . . . 92

Deep Flow . . . . . . . . . . . . . . . . 96

Model Sensitivity . . . . . . . . . . . . . . . . . 103

Deep Upwelling . . . . . . . . . . . . . . . . . . . 104

3.4 Mixing Rates and Flow in an Abyssal Canyon . . . . . . . . . 106

3.5 Discussion . . . . . . . . . . . . . . . . . . . 113

4 Conclusions $\quad 121$

4.1 Salt-Finger Mixing . . . . . . . . . . . . . . . . . . 123

4.2 Abyssal Dissipation . . . . . . . . . . . . . . . . . 125

$\begin{array}{ll}\text { References } & 129\end{array}$ 


\section{Chapter 1}

\section{Introduction}

The ocean is stratified by heat and salt. At the ocean's surface, these properties are controlled by the sun, wind and rain. If the ocean were stagnant, temperature and salinity conditions at the sea surface would not mix into the oceanic interior. The molecular diffusion of seawater would support a thin boundary layer between surface and abyssal properties. In truth, much of the ocean's depth is stratified. The gradients of heat, salt and density are strongly aligned in the vertical. Thus, the vertical, or diapycnal direction, is the direction of consequence for the production of buoyancy forces by mixing.

Questions regarding the role of vertical mixing in driving the ocean's circulation have persisted for many years. Some of the first dynamical ideas for both the abyssal (Stommel 1957, 1958) and the thermocline (Robinson and Stommel 1959) circulations relied heavily on the notion that a vertical diffusion of heat acts to balance the vertical advection occurring throughout the ocean's interior. Such a balance was invoked by Munk (1966), who examined vertical property distributions in the abyssal Pacific. Munk's calculations implied that a turbulent diffusivity of $k \sim \mathrm{O}\left(1 \mathrm{~cm}^{2} \mathrm{~s}^{-1}\right)$ is needed to balance an $\mathrm{O}(10 \mathrm{~Sv})$ global deep-water production through uniform upwelling, and this mixing-rate estimate became the benchmark for all assessments of oceanic mixing.

Direct observations of temperature structures at vertical scales of $\mathrm{O}(1 \mathrm{~cm})$ provide a means of estimating the rate of molecular dissipation, and these "microstructure" derived estimates of oceanic mixing-rates were introduced by Osborn and Cox (1972). Early microstructure observations from the Pacific (Gregg 
1980) and Atlantic (Gregg and Sanford 1980), the later including Gulf Stream and Bermuda Slope data, indicated that mixing levels in thermocline were an order of magnitude weaker than Munk's estimate. Additional microstructure observations have demonstrated that weak $\mathrm{O}\left(0.1 \mathrm{~cm}^{2} \mathrm{~s}^{-1}\right)$ mixing rates extend into the abyssal interior (Moum and Osborn 1986; Toole et al., 1994). While larger mixing rates have been observed in high-shear regions such as the Equatorial Undercurrents (Osborn 1980; Gregg et al., 1985) and the surface Ekman-layer (Oakey 1985), the vastness of the oceanic interior limits the global influence of these special regions.

The vertical mixing measured by microstructure is an intrinsically small-scale phenomenon, and its mechanics are not obviously related to larger-scale consequences. For this reason, the influence of vertical mixing on the ocean's circulation is not easily established. Ocean general circulation models (OGCMs) parameterize vertical mixing as a bulk eddy-diffusivity, often specified as a global constant. While these models are prone to producing spurious diapycnal mixing by laterally mixing across sloping isopycnals (Veronis 1975), their ability to resolve the global and decadal (centennial) scales of the wind driven and thermohaline circulations make OGCMs valuable for assessing the role of vertical mixing. In particular, Bryan (1987) assessed the implications of vertical mixing on meridional overturning and poleward heat-transport using both thermocline theory and an OGCM. Bryan used a closed-sector geometry and found that a realistic thermohalinecirculation results when $O\left(1 \mathrm{~cm}^{2} \mathrm{~s}^{-1}\right)$ diffusivities are specified. Such OGCMs with closed-sector basins have been modified to incorporate more sophisticated parameterizations of buoyancy forcing. Marotzke (1997) specified large diffusivities in thin boundary-layers around the basin, while Zhang et al. (1999) consider both "relaxation" and "mixed" boundary conditions for temperature and salinity. Both studies find that the overturning strength of the thermohaline cell is dependent on $k^{2 / 3}$. However, other OGCM studies using a global basin (Cox 1989; Toggweiler and Samuels 1995) find the structure and strength of the thermohaline flow is strongly influenced by the Antarctic Circumpolar Current (ACC). Toggweiler and Samuels (1998) find that the ACC drives a wind-powered thermohaline circulation without vertical mixing in the interior, consistent with predictions of scaling relations (Gnanadesikan 1999).

While studies using OGCMs have generally focused on the link between vertical 
diffusivity and the thermohaline circulation, the parameterizations of mixing in such models are poor. The influence of geographic and depth dependence of diapycnal mixing on circulation dynamics have not been thoroughly researched. Furthermore, the relationships between large-scale hydrography and buoyancy forcing remain vague. Observational programs that relate the mechanics of smallscale mixing with larger scales of motion remain necessary.

This thesis will address the implications of data collected during two such observational programs. The first case considered (chapter 2) involves doublediffusive mixing in the North Atlantic subtropical thermocline. The second case (chapter 3) considers turbulent mixing in the abyssal Brazil Basin. Both of these problems are attractive because they address poorly understood mixing-regimes. In both cases, the work will begin at the small scale with microstructure-derived mixing rates. The mixing rates will be used to derive the buoyancy fluxes that act between density layers of stratified flow. Diapycnal advection, the component of flow perpendicular to surfaces of potential density, will be of primary interest. Though a dynamically important quantity, diapycnal advection is a poorly constrained quantity in nearly all contexts of physical oceanography. It appears in the advective budgets for heat and salt, and also in the vorticity budget, where the divergence of diapycnal velocity acts as a vortex stretching mechanism. In this way diapycnal advection fundamentally links the small-scale processes of mixing to the larger-scale patterns of circulation.

In chapter 2, the case of mixing in the North Atlantic thermocline will be examined. This study is the consequence of a field program begun in 1992, The North Atlantic Tracer Release Experiment (NATRE). The experiment was conducted to document the details of mixing in the pycnocline and consisted of both a microstructure profiling and a tracer-release phase. The tracer work was conducted by Jim Ledwell and his colleagues, and is described by Ledwell et al. $(1993,1998)$. The results derived from the 2.5 year tracer evolution have greatly benefited the study of mixing in the ocean.

Prior to the tracer release result, the thermocline mixing-rates inferred from microstructure observations were questioned. The diffusivity estimates consistently indicated $k \leq 0.1 \mathrm{~cm}^{2} \mathrm{~s}^{-1}$, a value only 100 times larger than the molecular diffusion rate for heat. Furthermore, the thermocline estimates were an order 
of magnitude less than the diffusivity deduced by Munk (1966) for the abyssal ocean. Issues related to undersampling and intermittency of mixing were cited as reasons for microstructure estimates being low (Gibson, 1982). Additionally, the framework for estimating diffusivity from microstructure, first proposed by Osborn and Cox (1972), has been called into question (Davis 1994). However, after 30 months of evolution, the tracer cloud described by Ledwell et al. (1998) covered $\mathrm{O}(1000 \mathrm{~km})^{2}$ of the North Atlantic subtropical gyre, and the tracer result of $k \sim 0.1 \mathrm{~cm}^{2} \mathrm{~s}^{-1}$ showed little dependence on space or time. The tracer result has produced confidence in the $\mathrm{O}\left(0.1 \mathrm{~cm}^{2} \mathrm{~s}^{-1}\right)$ thermocline mixing-rates consistently estimated from microstructure.

In that the internal-wave climate of the thermocline is known to conform to the canonical description of the Garret and Munk spectrum (GM; Garrett and Munk 1975; Munk 1981), mixing in the thermocline has been interpreted in terms of wave instabilities and nonlinear interactions of the GM spectrum (Henyey et al. 1986; Polzin et al., 1995). However, the mechanism of double diffusion has long been identified as a possible additional contributor to mixing in the thermocline (Stern 1960). In particular, the so-called "salt finger" form of double diffusion is favored at mid-latitudes, where excess evaporation over precipitation causes the salinity to decrease with depth. The potential energy stored in the top-heavy salinity profile provides the energy source for mixing. Though the instability conditions of salt fingers have been understood for some time, an assessment of salt-finger mixing in the thermocline has been elusive. Since internal waves are an ubiquitous feature of the thermocline, most past work has assumed that all mixing in the pycnocline can be attributed to wave-field instabilities, even when the halocline favors the salt-finger instability.

The work presented in chapter 2 will generalize a thermodynamic model for velocity and shear microstructure which permits the signal of salt-finger mixing to be discerned in conditions where shear and internal waves are also active mixing mechanisms. While shear-produced turbulence acts to produce a downgradient density-flux, fingers flux density upgradient. The two processes compete to determine the direction of the density flux and the direction of the diapycnal advection. The data presented in chapter 2 show that for the shallow depths of the pycnocline $(z<400 \mathrm{~m})$ at the NATRE site, the divergence of the haline com- 
ponent of the buoyancy flux dominates over the thermal contribution, owing to the salt-finger enhancement of the salinity flux. This leads to a downward diapycnal advection that is not possible by turbulent mixing alone. Thus, salt-finger mixing represents a distinct mechanism of water-mass conversion. The results of chapter 2 provide observational evidence for the role of salt-fingers in modifying upper ocean temperature-salinity structure.

In chapter 3, the case of mixing in the abyssal Brazil Basin will be examined. Like the NATRE study, microstructure measurements were made to compliment a tracer-release experiment, the Brazil Basin Tracer Release Experiment (BBTRE, Polzin et al., 1997; Ledwell et al., 1999). This experiment was undertaken to document the rates and mechanisms of mixing in the abyssal ocean. The BBTRE microstructure and tracer observations constitute the most extensive abyssal-mixing data set ever collected. However, previous estimates of deep mixing-rates have been made. In particular, Hogg et al. (1982) deduced that a vertical diffusivity of $k \sim(2-4) \mathrm{cm}^{2} \mathrm{~s}^{-1}$ is needed to close the heat and mass budget of the Brazil Basin's densest water class. This estimate, and those for other abyssal basins, suggested that mixing rates in the deep could be orders of magnitude larger than in the thermocline.

The dense water of the abyss is far removed from contact with the atmosphere, where wind stress acts to input vorticity and drive circulation. Instead, the ocean's densest waters are formed in remote high-latitude seas and then advected to mid latitudes by boundary currents. In the abyssal interior, flow is supported by buoyancy forcing. These ideas were first proposed by Stommel (1957). Lacking specific details about how buoyancy forcing is distributed, Stommel and Arons (1960) examined a model where upwelling occurs uniformly throughout the abyss. While boundary currents are still recognized as a fundamental component of the deep circulation, ideas about buoyancy forcing have changed.

An association between enhanced mixing-rates and rough topography has been noted in prior reports (Polzin et al., 1997; Toole et al., 1997). These data suggest that mixing is related to internal-wave processes occurring well into the stratified water above the bathymetry. Bathymetric charts show systems of seamounts and other rough bathymetric features implying that enhanced buoyancy-forcing near abyssal topography will lead to complex and varied flow structures. 
The work presented in chapter 3 will assimilate observations of enhanced mixing near the Mid Atlantic Ridge with an inverse model describing the large-scale flow. This model constrains the diapycnal advection to conform in a self consistent way with both the thermodynamics dictated by the microstructure, and the vorticity, heat and mass budgets dictated by the hydrography. Steady geostrophic dynamics are assumed valid, and the contribution of vortex stretching by diabatic and adiabatic flow will be assessed.

Concluding remarks will be made in chapter 4 . There, the value of the findings from the two mixing regimes I examined will be discussed. This discussion will address implications for the work that extend beyond the specific regional nature of the two problems presented. In the remaining sections of this introductory chapter, some thermodynamical and dynamical considerations will be discussed.

\subsection{Thermodynamic Considerations}

At the largest scales, variance is input into the ocean through atmospheric forcing. The global budgets of two important variance quantities have been formulated; thermal variance (Joyce 1980) and mechanical energy (Lueck and Reid 1984). Both of these studies formulated the input of variance at large scales by air/sea interaction, and the removal of variance at small scales by mixing. At the smallest scales, variance is dissipated by the molecular diffusion of seawater. Thus, rates of variance dissipation are synonymous with the rates of small-scale mixing.

The thermodynamic equations for thermal variance and turbulent kinetic energy (TKE) are central to the mixing-rate estimation problem. These equations are derived from Reynolds decomposition of the Navier-Stokes and heat equations, as discussed by Monin and Yaglom (1971) and Tennekes and Lumley (1972). In standard tensor notation of the variables of fluid mechanics, the budget for thermal variance is

$$
\begin{gathered}
\frac{\partial}{\partial t} \overline{\theta^{2}}+\frac{\partial}{\partial x_{i}}\left(U_{i} \overline{\theta^{2}}+\overline{u_{i} \theta^{2}}-D_{\theta} \frac{\partial}{\partial x_{i}} \overline{\theta^{2}}\right) \\
=-2 \overline{u_{i} \theta} \frac{\partial \Theta}{\partial x_{i}}-\chi .
\end{gathered}
$$


Average quantities in (1.1) are denoted by upper case $\left(U_{i}, \Theta\right)$, and fluctuating quantities by lower case $\left(u_{i}, \theta\right)$. The terms that precede the equal sign represent the physical rearrangement of thermal variance in time and space. The three terms in parenthesis denote advection of thermal variance by the mean flow $\left(U_{i}\right)$ and the fluctuating flow $\left(u_{i}\right)$, and by molecular diffusion $D_{\theta}$. The source and sink terms follow the equal sign, with the rate of thermal variance extraction from the mean temperature field given by $2 \overline{u_{i} \theta}\left(\partial \Theta / \partial x_{i}\right)$, while the rate of dissipation by molecular diffusion $D_{\theta}$ is given by $\chi=2 D_{\theta} \overline{\left(\partial \theta / \partial x_{i}\right)^{2}}$. The budget for TKE is in many ways analogous,

$$
\begin{gathered}
\frac{\partial}{\partial t} \frac{1}{2} \overline{q^{2}}+\frac{\partial}{\partial x_{i}}\left(U_{i} \frac{1}{2} \overline{q^{2}}+\frac{1}{2} \overline{u_{i} u_{j} u_{j}}+\overline{u_{i} p}-\nu \frac{\partial}{\partial x_{i}} \frac{1}{2} \overline{q^{2}}\right) \\
=-\overline{u_{i} u_{j}} \frac{\partial U_{j}}{\partial x_{i}}-\epsilon-\frac{g}{\rho_{0}} \overline{w \rho}
\end{gathered}
$$

where $(1 / 2) q^{2}=(1 / 2)\left(u^{2}+v^{2}+w^{2}\right)$ is the TKE per unit mass and $\epsilon=$ $(1 / 2) \nu\left(\partial u_{i} / \partial x_{j}-\partial u_{j} / \partial x_{i}\right)^{2} \mathrm{~s}$ the dissipation rate. The spatial rearrangement terms in parenthesis denote advection of TKE by the mean flow $\left(U_{i}\right)$ and the fluctuating flow $\left(u_{i}\right)$, as well as the energy transfer by pressure work and viscous diffusion. The first of the source/sink terms in (1.2), $-\overline{u_{i} u_{j}}\left(\partial U_{j} / \partial x_{i}\right)$, represents the exchange of energy between the mean flow and the turbulence. For the class of turbulence of concern here, the smallest scales of the flow are damped by viscosity, and the cascade of energy is from the larger scales of the mean flow such that $-\overline{u_{i} u_{j}}\left(\partial U_{j} / \partial x_{i}\right)>0$, so that this term is referred to as the production of TKE. This is in contrast to so-called "geostrophic turbulence", where the direction of the energy transfer can be from small scales to large, as discussed by Starr (1968). Of the remaining terms, the viscous dissipation $\epsilon$ represents the loss of TKE to molecular viscosity. The final term represents the exchange of TKE with potential energy by buoyancy flux. In stably stratified conditions, the exchange is from kinetic energy to potential energy, so the buoyancy flux term acts as a sink.

Within the context of oceanic turbulence, the averaging associated with the Reynolds decomposition is applied over an ensemble of many observations. This procedure produces an energy balance that is both spatially and temporally averaged, and the following assumptions are applied: (1) the diffusive transport of 
thermal variance $D_{\theta} \partial\left(\overline{\left.\theta^{2}\right) / \partial x_{i}}\right.$ and the viscous transport of energy $\nu \partial\left(\overline{q^{2}} / 2\right) / \partial x$ are ignored as they scale as small compared to the production terms, (2) the triple correlation and pressure work advection terms are assumed to be spatially homogeneous and thus vanish when differentiated, as indicated by experimental work (Rohr et al., 1988; Itsweire et al., 1993), (3) the mean flow parameters are functions of the vertical coordinate only, and (4) over the scales of the temporal and spatial averaging, the distributions of thermal variance and TKE are stationary in time and homogeneous in space, so that the advective rates of change $\left(\partial / \partial t+U_{i} \partial / \partial x_{i}\right)$ vanish. These assumptions lead to greatly simplified variance budgets. A simplified budget for thermal variance was proposed by Osborn and Cox (1972),

$$
2(\overline{w \theta}) \Theta_{z}+\chi=0 .
$$

In this expression, the production of thermal variance by vertical flux is directly balanced by dissipation. Modeling the flux as a Fickian diffusion, the Osborn-Cox expression implies the thermal eddy-diffusivity relation $k_{\theta}=(1 / 2) \chi \Theta_{z}^{-2}$. The reduction of the (1.1) to (1.3) requires validity of numerous assumptions, all of which are difficult to justify for geophysical flows (Davis 1994). However, the production-dissipation balance stated in (1.3) can be derived from considerations independent of the thermal variance budget. This derivation is given by Winters and D'Asaro (1996), who show that the justified application of (1.3) relies on the definition of the mean temperature gradient $\Theta_{z}$. These authors propose $\Theta_{z}$ should be defined from a reference stratification exhibiting the minimum potential energy when compared to all possible rearrangements of a given profile of temperature. In practice, the "Thorpe sorting" technique (Dillon, 1984; after Thorpe 1977) commonly employed in microstructure analyses achieves the desired result.

A simplified form of (1.2) was considered by Osborn (1980),

$$
-(\overline{u w}) \bar{U}_{z}-\epsilon+\frac{g}{\rho_{0}} \overline{\rho w}=0 .
$$

The balance here is between the work done by Reynolds stress, the work done by buoyancy, and the dissipation. Normalized by the production term, this TKE budget is expressed as 


$$
\left(1-R_{f}\right)\left(-k_{\rho} N^{2}\right)+R_{f} \epsilon=0
$$

where $-k_{\rho} N^{2}$ is the Fickian buoyancy flux and $R_{f}$ is the mixing efficiency defined as the ratio of buoyancy consumption to kinetic-energy production (i.e., $R_{f}=$ $\left.-k_{\rho} N^{2}\left(\overline{u w} U_{z}\right)^{-1}\right)$. Equations (1.3) and (1.4) can be used to derive an expression for the ratio of the thermal to buoyancy eddy-diffusivities,

$$
\frac{k_{\theta}}{k_{\rho}}=\frac{\Gamma_{d}}{\Gamma_{0}}
$$

where two dimensionless groups have been identified: $\Gamma_{0}=R_{f}\left(1-R_{f}\right)^{-1}$ is related to the mixing efficiency and $\Gamma_{d}=\chi N^{2}\left(2 \epsilon \Theta_{z}^{2}\right)^{-1}$ is the scaled ratio of the dissipation rates.

In chapter 2 , a model for the parameter $\Gamma_{d}$ will be presented. The model will be used to examine the diffusivity ratio $k_{\theta} / k_{\rho}$. Evidence for double-diffusive processes will be sought when the implied diffusivity ratio differs from unity.

\subsection{Dynamical Considerations}

In the stratified ocean, the total vertical velocity consists of both adiabatic and diapycnal components. The adiabatic component is associated with flow along sloping density-surfaces. Diapycnal advection $\left(w_{*}\right)$ occurs when mixing produces a divergent flux of buoyancy. The diapycnal advection is linked thermodynamically to the divergence of buoyancy flux across a potential density or neutral surface.

The equation for advection across a neutral surface, neglecting terms that are nonlinear in thermodynamic variables, is (McDougall 1991),

$$
w_{*} N^{2}=-\frac{\partial J_{b}}{\partial z}
$$

where $z$ is the cross-isopycnal coordinate and $J_{b}$ is the buoyancy flux. An expression for the geostrophic vorticity equation may be defined for the layer bounded by two neutral surfaces (McDougall 1988)

$$
\beta v=\frac{f}{h} \underline{u} \cdot \nabla h+f \frac{\partial w_{*}}{\partial z}
$$


where $\beta$ is the planetary vorticity gradient, $h$ is thickness of a density layer, and $\underline{u}$ is the vector of lateral flow along the layer. The diapycnal advection may be expressed as the vertical gradient of dissipation using (1.4) and (1.6), and the vorticity equation (1.7) can be expressed as

$$
\beta v=\frac{f}{h} \underline{u} \cdot \nabla h+\left(\frac{R_{f}}{1-R_{f}}\right) \frac{\partial}{\partial z}\left(\frac{f}{N^{2}} \frac{\partial \epsilon}{\partial z}\right) .
$$

Thus for flow in geostrophic balance, the planetary vorticity is influenced by a diabatic-stretching term which can be expressed in terms of the vertical divergence of dissipated energy. In this manner, the geostrophic vorticity balance is influenced by the vertical mixing occurring at the smallest scales of fluid motion. This fundamental link between dissipation and the diabatic forcing of the flow serves as the philosophical motivation for the work of this thesis. Specifically, the above expressions demonstrate that knowledge of the vertical-mixing rate provides information on the three dimensional flow.

As a clearer way of demonstrating the importance of the diabatic forcing term in the vorticity equation, consider the following scaling relations. Using standard notation, we scale $(\underline{x}, z) \sim(L, H), \underline{u} \sim U, \beta \sim U / L^{2}$ and define the nondimensional parameters for the Rossby number $R o=U /(f L)$ and the deformation radius $R_{d}=N H / f$. The scaling for the term $\nabla h$ is taken from the geostrophic relation $f U \sim g^{\prime} \nabla h$, where the reduced gravity is $g^{\prime} \sim N^{2} H$. Additionally, we scale the energy dissipation term as $\epsilon \sim f E$, where $E$ is the scale of the kinetic energy associated with the forcing for the vertical mixing. This scaling for $\epsilon$ is proposed on solely dimensional grounds, noting that a time scale $f^{-1}$ is relevant for many dynamical regimes. Application of these scaling relations in (1.8) yields the following nondimensional equation,

$$
\beta v=\frac{L^{2}}{R_{d}^{2}} \underline{u} \cdot \frac{\nabla h}{h}+\Gamma_{0} \frac{E}{U^{2}} \frac{\partial}{\partial z}\left(\frac{L^{2}}{R_{d}^{2}} \frac{\partial \epsilon}{\partial z}\right),
$$

where the lower-case variables are now dimensionless. Thus, the ratio of the diabatic to adiabatic stretching is

$$
\frac{\text { diabatic stretching }}{\text { adiabatic stretching }}=\Gamma_{0} \frac{E}{U^{2}} \text {. }
$$


The parameter $\Gamma_{0}$ is the ratio of buoyancy flux to energy dissipation, and a value of $20 \%$ is justified for most turbulent mixing in the ocean (Moum 1996). Thus, the relative importance of the diabatic-forcing term will be dictated by the energy level of the process controlling the vertical mixing.

In chapter 3, mixing in the abyssal boundary layer will be examined. Observations suggest that dissipation is correlated with the magnitude of the barotropic tidal currents. In the most simple case of lee waves forced by the tide running over bathymetry, the energy in the internal-wave field available for mixing scales as $U_{\text {tide }}^{2}$. In the abyss where geostrophic flow is weak, typical velocities are $\mathrm{O}\left(1 \mathrm{~mm} \mathrm{~s}^{-1}\right)$. Barotropic tidal velocities are generally larger, say $\mathrm{O}\left(1 \mathrm{~cm} \mathrm{~s}^{-1}\right)$. Thus a ratio of $\mathrm{O}(1)$ for the diabatic to adiabatic vorticity-forcing is easy to justify, demonstrating the strong dynamical link to circulation and mixing in the abyss. 


\section{Chapter 2}

\section{The Contribution of Salt Fingers to Vertical Mixing in the North Atlantic Tracer Release Experiment}

\section{$2.1 \quad$ Introduction}

Dissipation rates of thermal variance $(\chi)$ and turbulent kinetic energy (TKE, $\epsilon$ ) are used to infer diapycnal fluxes of heat, salt and density in the ocean. The divergence of the density flux will dictate the strength of the diapycnal advection. Further dynamics are set by the vertical divergence of the diapycnal advection, which provides the vorticity forcing in the ocean's interior. Thus, in a fundamental way, the general circulation of the ocean is influenced by the second derivatives of the dissipation rates with respect to the vertical. This work will examine the problem of estimating the diapycnal advection from observations of $\chi$ and $\epsilon$ in a region influenced by the salt-finger form of double-diffusive convection.

Many regions of the world ocean are characterized by haline stratification that is top heavy with respect to density. Such regions include the Mediterranean outflow, the western tropical North Atlantic, and the "Central Waters" of the subtropical gyres (Schmitt 1994). The unstable potential energy stored in the topheavy salinity stratification may be released in small-scale convection cells known as "salt fingers". The vertical fluxes produced by the finger instability act to diffuse concentrations of heat and salt down their mean gradients. While convecting 
plumes (fingers) generally preserve their salinity variance, thermal variance is lost to the molecular conduction of heat across individual cell boundaries. Hence, fingers act to vertically transport salinity more efficiently than heat. The haline component of the buoyancy flux exceeds the thermal component, resulting in an up-gradient flux of density. Mixing by salt fingers may be modeled in terms of separate eddy diffusivities for heat $\left(k_{\theta}\right)$, salt $\left(k_{s}\right)$ and density $\left(k_{\rho}\right)$. While the finger fluxes of heat and salt are both down their respective gradients, with $k_{s}>k_{\theta}>0$, the finger flux of density is up-gradient so that $k_{\rho}<0$. This up-gradient flux of density has been shown to form and maintain a thermohaline staircase, in which a series of well-mixed layers are separated by sharp interfaces (Stern and Turner 1969). Such staircases have been observed at the Mediterranean outflow, the Tyrrhenian Sea, and in the tropical Atlantic near Barbados (Schmitt et al., 1987). The existence of a thermohaline staircase is taken to be a strong indicator of salt-finger mixing.

However, staircases are not always found in open-ocean regions having fingerfavorable stratification. In general, the up-gradient density flux induced by salt fingers will be opposed by down-gradient turbulent fluxes produced by internal wave breaking. Unlike salt-finger mixing, the high Reynolds number turbulence occurring in the ocean produces fluxes of heat and salt that are uninfluenced by differences in molecular properties, resulting in uniform diffusivities among scalars (i.e., $k_{\theta}=k_{s}=k_{\rho}$ ). Thus, in a region experiencing both salt-finger and turbulent fluxes, the magnitude and direction of the net buoyancy-flux is then determined by the competition between the two processes. To properly determine the buoyancy flux in these regimes, a means of assessing both the relative occurrence and magnitude of the two processes is needed. The need for a procedure allowing calculation of the net $k_{\rho}$ in a region with both turbulence and salt fingers serves as the primary motivation for this study.

The work presented here will rely on observations of microscale dissipation obtained from the free falling High Resolution Profiler (HRP, Schmitt et al., 1987), as a means of assessing the diapycnal fluxes occurring in the thermocline at the NATRE site. A previous comparison of microstructure-derived diffusivities (Toole et al., 1994) and tracer-derived diffusivities (Ledwell et al., 1993) has indicated general agreement. This paper will strive to identify the turbulent and salt-finger 
contributions to the net diffusivities. Furthermore, we will allow for the possible elevation of the haline diffusivity over the thermal diffusivity, as well as the possibility for a negative density diffusivity. We will establish a means of identifying both the relative frequency and magnitude of salt-finger and turbulent dissipation events utilizing several nondimensional parameters available from quantities measured by the HRP. The density ratio, $R_{\rho}=\left(\alpha \Theta_{z}\right)\left(\beta S_{z}\right)^{-1}$, is the parameter that dictates a system's susceptibility to double-diffusive instability. The salt-finger instability is permitted if $1<R_{\rho}<100$ (Schmitt 1979a). However, in an ocean constantly perturbed by internal wave strain, modes of instability with characteristic period longer than that of the wave field $\left(2 \pi N^{-1}\right)$ may fail to grow. For this reason Schmitt and Evans (1978) suggest only modes with growth rates near $N$ will become strongly established, this being true if $1<R_{\rho}<2$. A second parameter, the gradient Richardson number, $R i=N^{2} U_{z}^{-2}$, measures a system's susceptibility to shear $\left(U_{z}\right)$ instability. While much work has focused on the $R i<0.25$ instability condition, Polzin (1996) found a general increase in turbulent dissipation when $R i<1$. Finally, we will use the nondimensional ratio of the dissipation rates, $\Gamma=\left(\chi N^{2}\right)\left(2 \epsilon \Theta_{z}^{2}\right)^{-1}$. This quantity, "the dissipation ratio", is related to both mixing efficiency $\left(R_{f}\right)$ and the ratio of the diffusivities for heat and buoyancy. Models for this parameter have been formulated for both turbulence (Oakey 1985) and salt-finger mixing (Hamilton et al. 1989; McDougall and Ruddick 1992). Ruddick et al. (1997) have examined dissipation at the NATRE site in a similar manner, using $R_{\rho}, \Gamma$, and the nondimensional TKE dissipation parameter $\epsilon\left(\nu N^{2}\right)^{-1}$ (a Reynolds number). Ruddick et al. (1997) failed to find a significant signal of salt fingering. However, the gradient Richardson number provides a strong constraint for identifying turbulence produced by shear instability, and we will show that the parameter family of $R_{\rho}, R i$, and $\Gamma$ is sufficient for isolating the signal of salt fingers even in conditions where turbulence is also occurring.

We will begin by describing some qualitative evidence for salt fingers at the NATRE site (section 2.2). Quantitative evidence for salt-finger mixing in terms of dissipation data is presented in section 2.3. We give a description of HRP data (2.3.1), present a model of the dissipation ratio (2.3.2), discuss the method of analysis (2.3.3), discuss the statistical treatment of dissipation data (2.3.4), 
and present our results (2.3.5). The results of the $\left(R_{\rho}, R i, \Gamma\right)$ parameter analysis are then applied to the NATRE profile data to estimate the net diffusivities of heat and salt (section 2.4.1) and the diapycnal advection (2.4.2). We present a discussion of this material in section 2.5.

\subsection{Qualitative Evidence for Salt Fingers at the NATRE Site}

The North Atlantic Tracer Release Experiment (NATRE) was conducted in the Canary Basin of the eastern North Atlantic. A 150-station microstructure survey of the release site was completed prior to the tracer release phase of the experiment, discussed by Ledwell, Watson and Law (1993, 1998). Measurements were made using a free falling, autonomously profiling instrument, the High Resolution Profiler (HRP). The HRP produces measurements of temperature, conductivity and velocity at both finescale $(\mathrm{O}(1 \mathrm{~m}))$ and microscale $(\mathrm{O}(1 \mathrm{~cm}))$ resolution. Measurements of optical microstructure were also made using a shadowgraph imaging system. Descriptions of the HRP and all of its component instruments, including the shadowgraph, are given by Schmitt et al. (1988).

The NATRE site rests within the Central Waters of the North Atlantic subtropical gyre (figure 2.1). Several studies (Schmitt and Evans 1978; Schmitt 1981; Schmitt 1990) have argued that salt fingers contribute to the vertical mixing in this region. In particular, these studies argue that finger mixing should dominate turbulent mixing if $R_{\rho} \leq 2$. Indeed, this describes the density ratio structure in the upper $500 \mathrm{~m}$ of the NATRE site thermocline (figure 2.2). However, no permanent thermohaline staircase occurs at the NATRE site. This suggests that the finger contribution to the vertical buoyancy-flux is insufficient for maintaining a series of well mixed layers and sharp interfaces. If turbulent fluxes produced by shear instability of internal waves are large enough (and frequent enough) to overcome the finger fluxes, the net density-flux may be down-gradient. Polzin (1996) has found a clear connection between mixing and shear instability at the NATRE site. His work indicates that exceptional dissipation generally occurs when the local Richardson number $\left(R i=N^{2} U_{z}^{-2}\right)$ becomes less than unity. To what extent these turbulent dissipation events compare to the dissipation of salt fingers must 


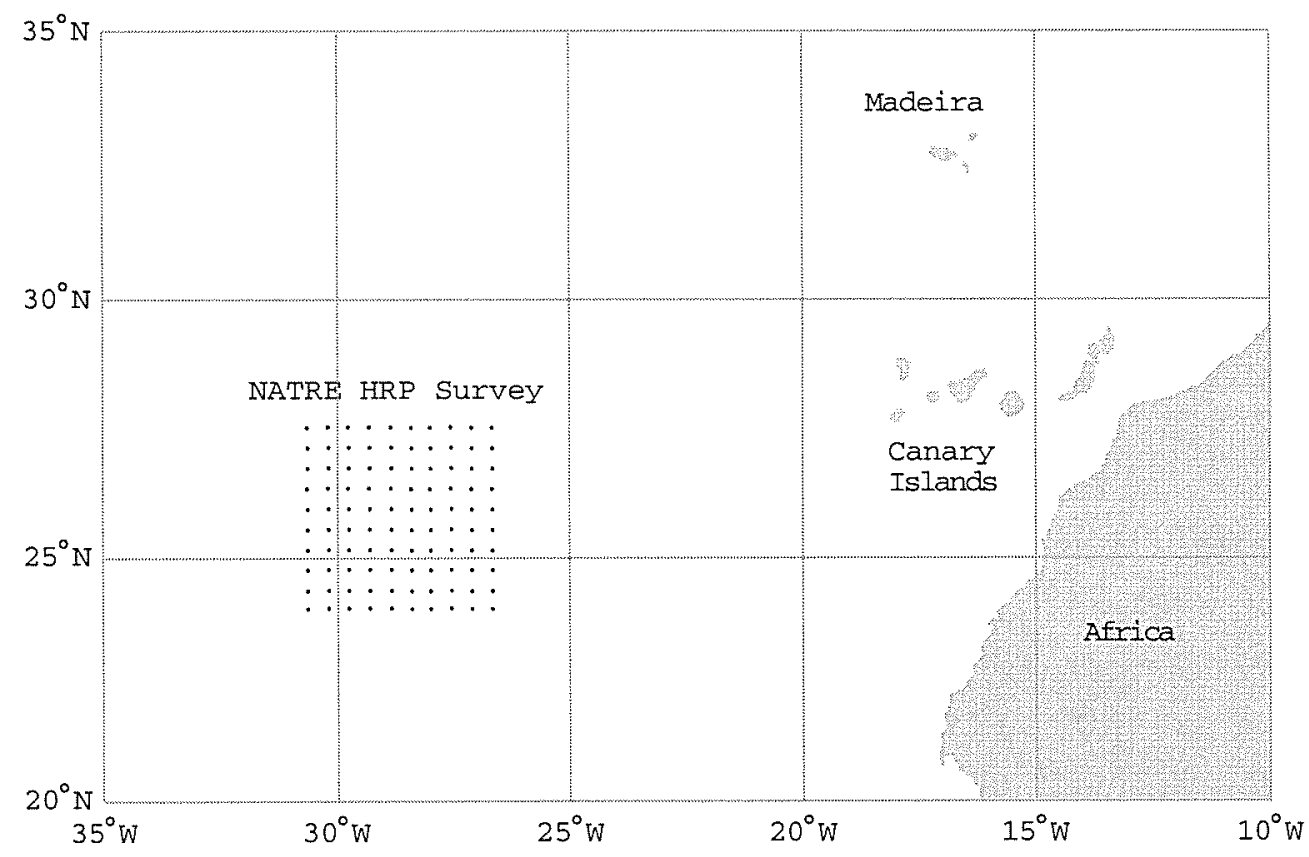

Figure 2.1: Location of the NATRE HRP survey. Over 150 dives were completed over 26 days. The survey consisted of the 100 station grid spanning the $(400 \mathrm{~km})^{2}$ region shown. An additional 50 stations were tightly centered about $\left(26^{\circ} \mathrm{N}\right.$, $28^{\circ} \mathrm{W}$ ). The HRP survey was completed two weeks prior to the tracer-release phase of the experiment. 
be explored further.

Despite the lack of a thermohaline staircase, optical structures recorded during two shadowgraph profiles give qualitative evidence of salt-finger activity. Thin filament-like optical structures (figure 2.3) occurred from the bottom of the mixed layer $(z \sim 150 \mathrm{~m})$ to the base of the thermocline $(z \sim 1000 \mathrm{~m})$. These features are most abundant just below the mixed layer, occurring in patches with several meter vertical extent, with gaps between patches of 5 to $10 \mathrm{~m}$. Patches containing filament structures become more sparse at at greater depth, but generally occur with a frequency of 2-4 patches for every $40 \mathrm{~m}$. While all possible orientations were encountered, filaments in the form of laminae tilted 10 to 20 degrees from horizontal were most frequently observed (figure 2.3a). These structures are identical to those previously observed by Kunze et al. (1987) in a thermohaline staircase. As was the case with the staircase observations, the laminae at the NATRE site are characterized by cross-filament wavelengths of 0.5 to $1 \mathrm{~cm}$. Kunze (1990) identified these structures as salt fingers that have been tilted by shear. Other classes of optical structures observed include sharp interfaces, isotropic features, and billows.

We regard the abundance of thin tilted laminae at the NATRE site as suggestive evidence for salt fingers. To quantitatively assess the frequency and strength of salt fingers at the NATRE site, we rely on estimates of $\chi$ and $\epsilon$ derived from HRP microstructure measurements.

\subsection{Quantitative Assessment of Salt-Finger Mixing}

\subsubsection{Description of Data}

Data from the NATRE HRP survey will provide the foundation for this study. However, with the intent of making our study more general, we have supplemented the NATRE data with data from a second HRP Survey. These data come from

field work conducted in the northeast subtropical Pacific at Fieberling Guyot. Data from the Fieberling survey (TOPO) is discussed by Toole et al. (1997a) and Kunze and Toole (1997). In the present study, TOPO data are included as 


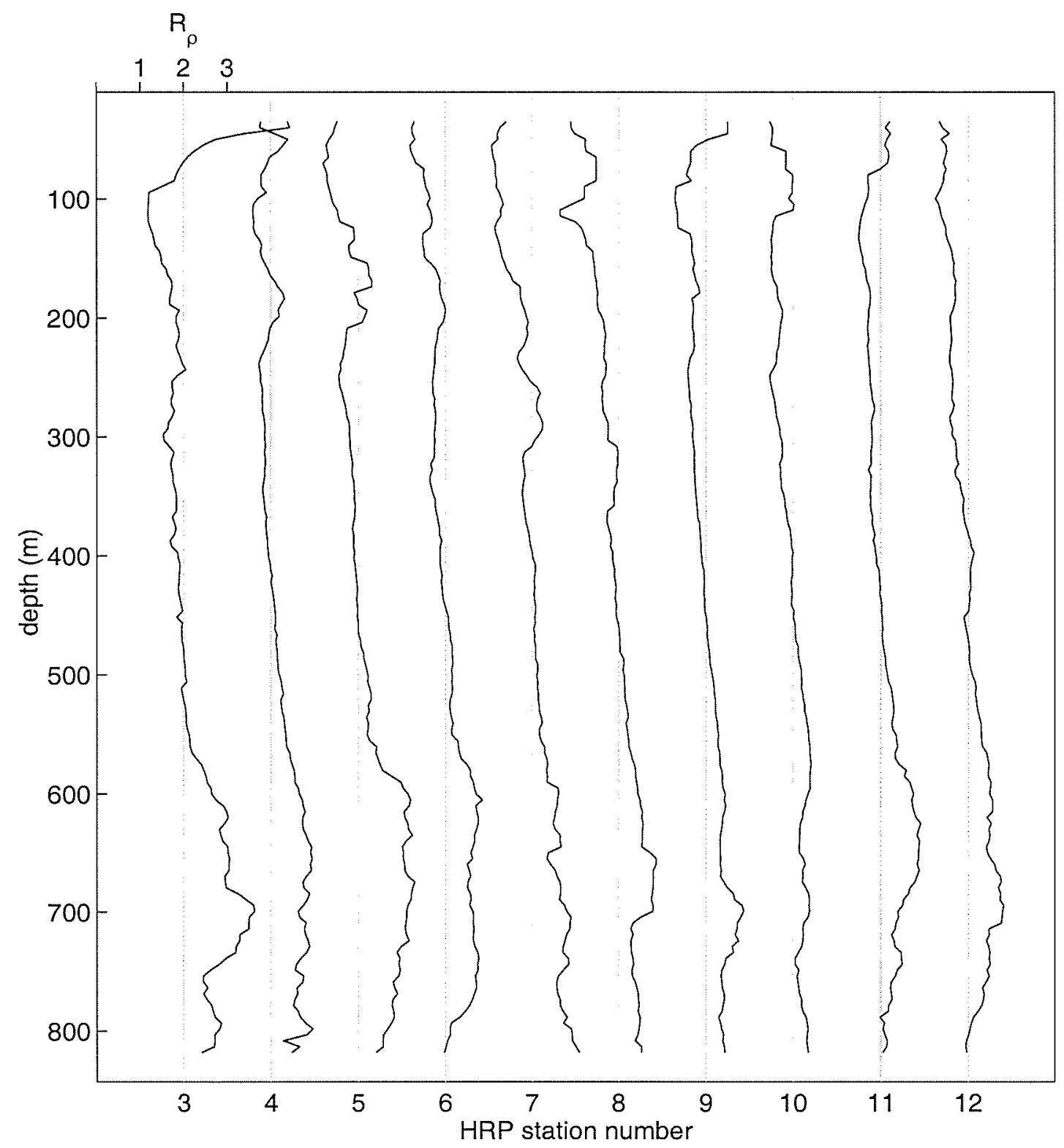

Figure 2.2: A section showing 10 profiles of the density ratio, $R_{\rho}=\alpha\left\langle\Theta_{z}\right\rangle$ $\left(\beta<S_{z}>\right)^{-1}$. The gradients were calculated using a 5 -m scale, smoothed using a 50-m running average. Stations $3-12$ comprise the meridional section at the western edge of the survey. Each successive station is offset two $R_{\rho}=2$ units and the reference value $R_{\rho}=2.0$ is shown for each profile. There is large variability in the mixed layer and beneath $z=600 \mathrm{~m}$ due to intrusive features. 

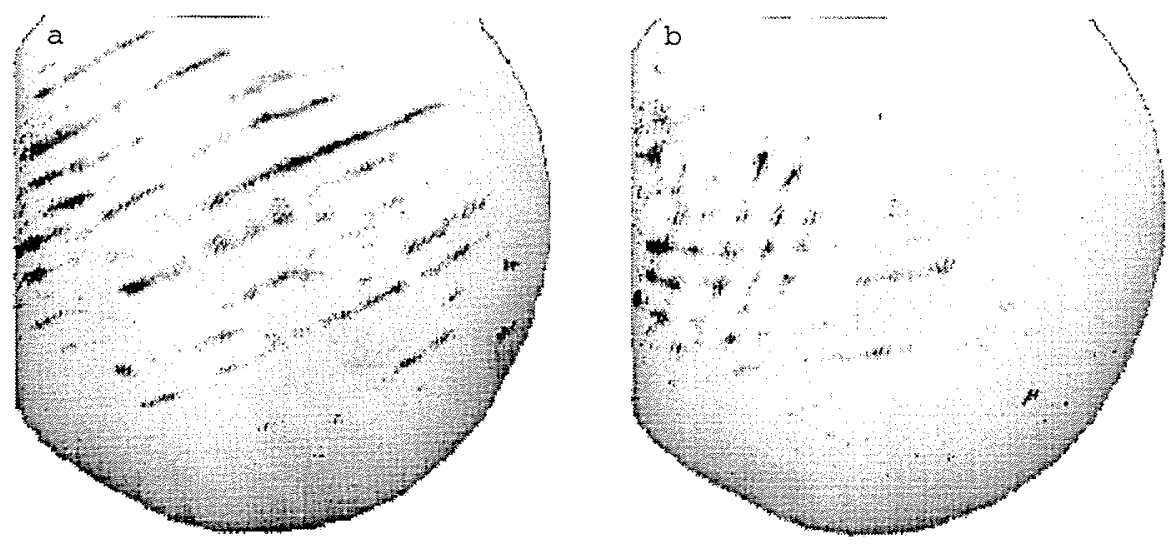

Figure 2.3: Shadowgraph images of optical microstructure that were obtained during the NATRE HRP survey. (Shadowgraph image intensity is proportional to the Laplacian of the refractive index of light. The negative of each original image is shown here.) The tilted laminae shown here are observed throughout the thermocline. The circular window has a diameter of $10 \mathrm{~cm}$, and the optical features have a characteristic wavelength of $0.5-1.0 \mathrm{~cm}$. Laminae tilted 10 to 20 degrees from the horizontal (a) were the most frequently observed orientation, although filaments with vertical alignment (b) were also observed. The images shown here were obtained near $300-\mathrm{m}$ depth. 
a means of introducing data from a double-diffusively stable (hereafter, doubly stable) stratification regime. While dissipation occurring in the salt-finger regime may be attributable to a combination of turbulence and fingers, dissipation in the doubly stable regime can only be attributed to turbulence. By regarding the features of turbulent dissipation in the doubly stable regime as a null hypothesis, we can objectively assess the dissipation observed in finger-favorable data.

The profile data from NATRE typically extends to $2000 \mathrm{~m}$. These profiles are characterized by a deep mixed layer (80-150 $\mathrm{m}$ thick) capping the finger-favorable thermocline. Below the thermocline, intrusive features exist with both "diffusive" favorable (the form of double diffusion with cold-fresh water over warm-salty) and doubly stable character. The TOPO data can be broken into two classes. The data collected above the seamount summit are characterized by high shear and weak stratification in the presence of thermohaline interleaving. The data collected on the seamount flanks are characterized by lower levels of shear and stronger stratification. In particular, these two classes have heterogeneous shear statistics, with shear levels at the summit exceeding those at the flank by a factor of two. For this reason, we will treat these two classes of TOPO data separately in the analysis that follows. While the stratification at the TOPO site was generally doubly stable, some double-diffusive favorable patches were also present.

Initial processing of all HRP data results in estimates of all conventional (e.g., $\Theta, S, U, V)$ and microstructure quantities at 0.5-m intervals. A detailed description of the algorithms used for this initial stage of data analysis can be found in Polzin and Montgomery (1996). Dissipation rates are calculated from observations of thermal and velocity microstructure using the relations $\chi=2 \kappa\left(3 \bar{\theta}_{z}^{2}\right)$ and $\epsilon=\nu(15 / 4)\left(\overline{u_{z}^{2}+v_{z}^{2}}\right)$, where $\kappa$ and $\nu$ are the molecular values of thermal diffusion and viscosity. The factor of 3 in the $\chi$ expression and the factor of $3.75 \mathrm{in}$ the $\epsilon$ expression come from an assumption of small-scale isotropy. Observations supporting the isotropy relations have been made for turbulence (Yamazaki and Osborn 1990) as well as salt fingers (Lueck 1987). Numerical simulations of salt fingers also indicate isotropy for the thermal gradients (Shen 1995). We note that shadowgraph images associated with fingers show significant structural coherence at $\mathrm{O}(1 \mathrm{~mm})$ scales. Since the shadowgraph measures the Laplacian of refractive index, the images tend to emphasize the smallest scales which are mainly influenced 
by salinity microstructure (Kunze 1990). Thus, small-scale thermal gradients may adhere to the isotropic relationship, while anisotropic salinity structures bias the shadowgraph images.

Finestructure gradient quantities, particularly $R_{\rho}$ and $R i$, will be used extensively in the analysis that follows. To estimate the vertical gradients of scalars, we have used the slope of a linear fit over a 5-m segment, centered at each 0.5 $\mathrm{m}$ interval. The 5-m scale was chosen as a suitable trade off between the need for high vertical resolution and statistically reasonable regression estimation. The magnitudes of all 5-m scalar gradients were compared to their associated standard error. Gradient quantities with standard errors larger that twice their magnitude were excluded from the analysis. This resulted in roughly a $5 \%$ data loss, mostly from noisy $N^{2}$ estimates. Figure 2.4 shows typical profiles from the two HRP surveys used for this study. Data from the TOPO survey are shown in figure 2.4a (a seamount summit profile) and figure $2.4 \mathrm{~b}$ (a seamount flank profile). A profile from NATRE is shown in figure 2.4c.

\subsubsection{The Dissipation Ratio Model}

Mixing by turbulence and salt fingering has traditionally been modeled by the production-dissipation balances for thermal variance (Osborn and Cox 1972) and TKE (Osborn 1980). These balances, in a form relevant to the average over an ensemble of many patches (denoted by $<\cdot>$ ), are given by

$$
\begin{aligned}
& \left(1-R_{f}\right)\left(-k_{\rho}<N^{2}>\right)+R_{f}<\epsilon>=0 \\
& 2\left(-k_{\theta}<\Theta_{z}>\right) \cdot<\Theta_{z}>+<\chi>=0 .
\end{aligned}
$$

In these expressions, $N^{2}$ and $\Theta_{z}$ are the vertical gradients of buoyancy and potential temperature, $k_{\rho}$ and $k_{\theta}$ are the vertical eddy diffusivities of buoyancy and temperature, and $R_{f}$ is the mixing efficiency. The mixing efficiency, or flux Richardson number, dictates the fraction of Reynolds stress production that is converted to potential energy flux (i.e., $R_{f}=-k_{\rho}<N^{2}>\left(\overline{u^{\prime} w^{\prime}}<U_{z}>\right)^{-1}$ ).

The buoyancy flux can be written in terms of the fluxes of heat and salt, 

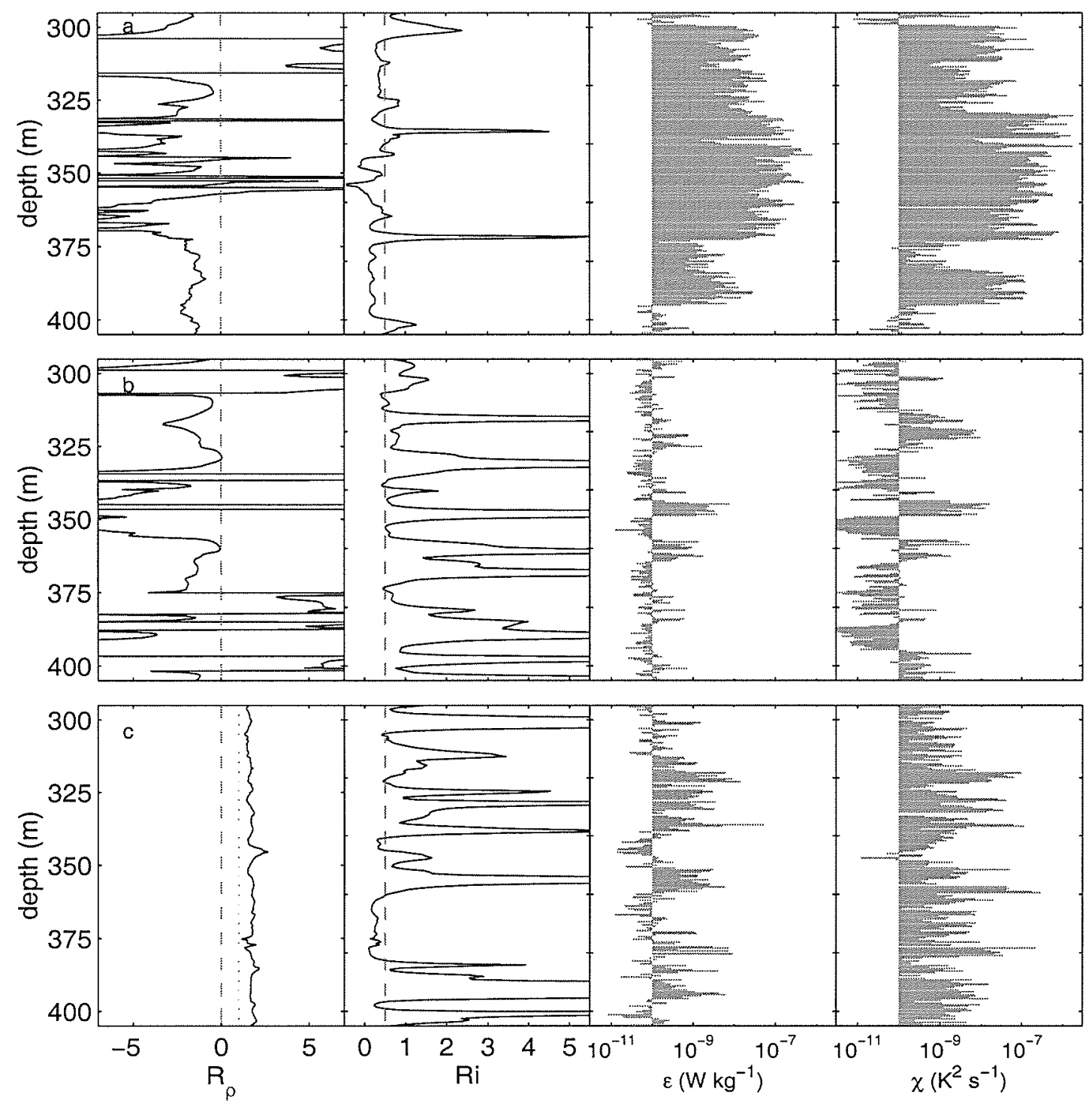

Figure 2.4: Characteristic profiles of $R_{\rho}, R i, \epsilon$ and $\chi$ from the three HRP data groups used in this study. (a) Data from above the summit of Fieberling Guyot collected as part of the TOPO HRP survey. (b) Data from the flank of Fieberling Guyot, about $20 \mathrm{~km}$ off the axis of the summit. (c) Data from the NATRE HRP survey. The TOPO site is characterized by predominantly doubly stable stratification $\left(R_{\rho}<0\right)$. In each profile of $R i$, a reference value of 0.5 is shown as the dashed vertical line. The high occurrence of low $R i$ above the seamount distinguishes the summit profiles (a) from those above the seamount flanks (b). 


$$
\begin{aligned}
-k_{\rho}<N^{2}> & =g\left(\alpha\left(-k_{\theta}<\Theta_{z}>\right)+\beta\left(-k_{s}<S_{z}>\right)\right) \\
& =\quad g \alpha\left(-k_{\theta}<\Theta_{z}>\right)\left(1-r^{-1}\right) .
\end{aligned}
$$

where we have defined the heat/salt buoyancy-flux ratio $r=\left(k_{\theta} / k_{s}\right) R_{\rho}$. In all cases, vertical scalar fluxes have been written in a Fickian form, with the eddy diffusivities being positive for down-gradient flux. Furthermore, we have carried a separate diffusivity for each scalar. In the case of salt fingering, not only do we expect the diffusivities to be different, but also that the salt flux can dominate the buoyancy flux so that $k_{\rho}<0$.

A general relation involving the ratio of thermal and buoyancy diffusivities can be derived using (2.1), (2.2) and (2.3) with $N^{2}=g \alpha \Theta_{z}\left(1-R_{\rho}^{-1}\right)$,

$$
\begin{aligned}
\Gamma & =\left(\frac{R_{f}}{1-R_{f}}\right) \frac{k_{\theta}}{k_{\rho}} \\
& =\left(\frac{R_{f}}{1-R_{f}}\right)\left(\frac{R_{\rho}-1}{R_{\rho}}\right)\left(\frac{r}{r-1}\right) .
\end{aligned}
$$

The nondimensional parameter $\Gamma$ (Oakey 1985) is the scaled ratio of the dissipation rates,

$$
\Gamma=\frac{\left.<\chi><N^{2}\right\rangle}{2<\epsilon>\left\langle\Theta_{z}\right\rangle^{2}} .
$$

We will refer to $\Gamma$ as the "dissipation ratio", although it has been referred to as "the mixing efficiency" by many investigators. While $\Gamma$ is related to the mixing efficiency, it is more generally related to the ratio of heat and buoyancy diffusivities. Oakey (1985) considered the case of turbulent mixing and derived

$$
\Gamma^{(t)}=\frac{R_{f}}{1-R_{f}} .
$$

This expression can be obtained from (2.4) by setting $k_{\theta}=k_{\rho}$, so that $r=R_{\rho}$. The superscript $(t)$ is used to denote that the relation is valid when turbulence is the sole dissipative mechanism. Thus, within the context of turbulent mixing, the dissipation ratio is related in a simple manner to the mixing efficiency $R_{f}$. We note that expression (2.6) can be restated as $\Gamma^{(t)}=\left(k_{\rho}\left\langle N^{2}\right\rangle\right) /\langle\epsilon\rangle$. Therefore, while $R_{f}$ is the ratio of potential energy gain to kinetic energy input, $\Gamma^{(t)}$ is the ratio of potential energy gain to kinetic energy loss. Laboratory experiments have demonstrated that the mixing efficiency of turbulence is small, with estimates 
ranging from $R_{f}=0.05$ (Huq and Britter 1995) to $R_{f}=0.20$ (Rohr et al. 1984). In terms of the oceanographic application of $(2.1), \Gamma^{(t)}=0.2$ is often used (Moum 1996).

Hamilton et al. (1989) and McDougall and Ruddick (1992) considered the case of salt-finger mixing and derived

$$
\Gamma^{(f)}=\left(\frac{R_{\rho}-1}{R_{\rho}}\right)\left(\frac{r}{1-r}\right)
$$

with the superscript (f) used to denote dissipation by salt fingers. This expression is also a special case of (2.4) where the Reynolds stress production $\left(P=\overline{u^{\prime} w^{\prime}} U_{z}\right)$ is zero such that $\lim _{P \rightarrow 0} R_{f}\left(1-R_{f}\right)^{-1}=-1$, as is the case for convection with a TKE balance of $-k_{\rho}\left\langle N^{2}\right\rangle=\langle\epsilon>$. Thus, for salt-finger mixing, $\Gamma$ is (minus) the ratio of the thermal to buoyancy diffusivity (i.e., $\Gamma^{(f)}=-k_{\theta} / k_{\rho}$ ). The size of this ratio is set by both the density ratio and the buoyancy-flux ratio of the fingers. The plausible range of the buoyancy-flux ratio $r$ is known from theory (Stern 1975; Schmitt 1979a), laboratory work (Turner 1967; Schmitt 1979b; McDougall and Taylor 1984; Taylor and Bucens 1989), and numerical simulations (Shen 1993,1995). This collection of work suggests $0.4<r<0.7$.

Figure 2.5 presents the plausible range of the nondimensional parameters of the salt-finger and turbulence models. Results from laboratory studies were used to plot the mixing efficiency of turbulence (5a) and the buoyancy-flux ratio of salt fingers (5b). These numbers were used to compute the dissipation ratio models for turbulence and salt fingers $(5 \mathrm{c})$. The value $\Gamma^{(t)}=0.2$ is shown as representative of the turbulence model, with a plausible range shown as $0.05<\Gamma^{(t)}<0.25$. The plausible range of the salt-finger dissipation ratio is shown with a $99 \%$ confidence band determined from the density-ratio binned statistics of $r$.

\subsubsection{Method of Analysis}

Our primary investigation of the dissipation rates will be done using the dissipation ratio $\Gamma$. The existence of simple models for $\Gamma$ in cases of turbulent and saltfinger dissipation give this parameter merit. However, two issues detract from this parameter's apparent usefulness. First, differences as small as a factor of two distinguish the value of $\Gamma$ between the two processes. This obstacle can be 

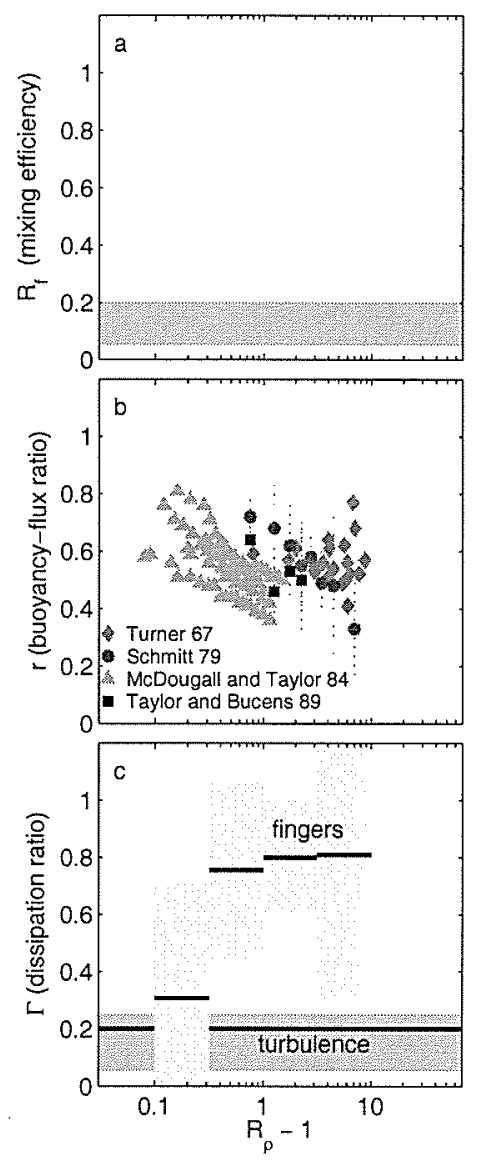

Figure 2.5: The plausible range of three nondimensional parameters for fingerfavorable stratification $\left(R_{\rho}>1\right)$. The logarithmic axis for $R_{\rho}-1$ is useful because the distribution of finger-favorable $R_{\rho}$ is approximately lognormal about $R_{\rho}=2$. (a) The mixing efficiency of turbulent mixing $\left(R_{f}\right)$. Laboratory data supports the range $0.05<R_{f}<0.20$. (b) The buoyancy-flux ratio of salt fingers $r$ from laboratory data. (c) The range of the dissipation ratio for turbulence and salt fingers are shown. The turbulence model was calculated using $\Gamma^{(t)}=R_{f}(1-$ $\left.R_{f}\right)^{-1}$, with $\Gamma^{(t)}=0.2$ taken as the nominal value. The finger model was computed from the laboratory data for $r$, with average values of $r$ binned by $R_{\rho}$. The $99 \%$ confidence band is shown for the finger model while the range for the turbulence model is dictated by $0.05<R_{f}<0.20$. 
overcome by incorporating large numbers of dissipation observations into each estimate of $\Gamma$ to reduce error bars enough to resolve such a subtle parameter range. This is a serious consideration since $\Gamma$ is the ratio of four noisy variables. Second, given an ensemble of dissipation observations, only a fraction of the data may be representative of mixing events appropriately modeled by (2.1) and (2.2). This issue must receive careful attention.

The dissipation rates $\epsilon$ and $\chi$ are computed by integrating the observed shear and thermal variance residing at scales between about 1 and $50 \mathrm{~cm}$. Variance at these scales originates from diabatic, irreversible processes. The processes of turbulence and salt fingering are among these, producing fluxes of heat, salt, and buoyancy that irreversibly alter the local temperature, salinity, and density finestructure. However, it is plausible that internal wave and molecular processes may also account for variance at small enough scales to influence dissipation estimates, and these set the oceanic background levels of the dissipation rates. This type of oceanic "noise" is not appropriately modeled by (2.1) and (2.2). Similarly, the noise of the sensors and associated electronics, though low for the HRP (Polzin and Montgomery 1996), will contribute to uncertainty in $\epsilon$ and $\chi$.

In the work presented here, we seek to attribute observations of irreversible microstructure to salt fingers or turbulence. We have attempted to rule out the influence of noisy dissipation estimates by only examining dissipative events of higher magnitude, while still retaining enough data to uncover a potentially subtle signal of salt fingers. To do this, we have used the combined data set involving observations from both NATRE and TOPO surveys. This combined dissipation record was then examined in terms of various upper thresholds of dissipation rate magnitude, this being done separately for observations occurring in doubly stable and finger-favorable patches.

We have found that exceptional levels of finger-favorable $\chi$ are associated with bimodal $\epsilon$ statistics. In particular, we have examined the distribution of $\epsilon$ data using a threshold defined as $\chi>\chi_{75} \sim 1 \times 10^{-9} \mathrm{~K}^{2} \mathrm{~s}^{-1}$ : the upper 75 percentile of the combined $\chi$ record. The statistical distribution for $\epsilon\left(\chi>\chi_{75}\right)$ is shown in figure 2.6. The finger-favorable data (figure 2.6a) seems to have a primary mode at $\epsilon \sim 2 \times 10^{-10} \mathrm{~W} \mathrm{~kg}^{-1}$ with the secondary mode occurring near $\epsilon \sim 1 \times 10^{-9} \mathrm{~W} \mathrm{~kg}^{-1}$. A simple statistical test for bimodality (Haldane 1952) indicates that the apparent 
antimode at $\epsilon \sim 5 \times 10^{-10} \mathrm{~W} \mathrm{~kg}^{-1}$ is significant to the 0.15 level (i.e., significant at the $85 \%$ confidence level). In contrast to the finger-favorable data, the associated distribution of doubly stable $\epsilon$ data lacks bimodal character (figure 2.6b). Both turbulence and salt fingers act as dissipative mechanisms in the finger-favorable regime, while only turbulence acts in the doubly stable regime. The existence of bimodal $\epsilon$ in only the finger-favorable regime suggests that the two modes are associated with the two processes.

To investigate the character of the finger-favorable data more thoroughly, we have examined the $\epsilon\left(\chi>\chi_{75}\right)$ population in terms of the stability parameters $R_{\rho}$ and $R i$. Figure 2.7 shows the distribution of $\epsilon\left(\chi>\chi_{75}\right)$ data after being partitioned into four subsections of $\left(R_{\rho}, R i\right)$ data space. When the portion of parameter space having $R i>1$ is considered, the low- $\epsilon$ mode dominates. This is particularly true when $R_{\rho}<2$ (figure 2.7a), this low density ratio range having about twice the number of $\epsilon\left(\chi>\chi_{75}\right)$ events as $R_{\rho}>2$ (figure 2.7b). In the portion of parameter space with $R i<1$ (figures $2.7 \mathrm{c}$ and $2.7 \mathrm{~d}$ ), the high- $\epsilon$ mode is apparent at both large and small values of $R_{\rho}$. However, in the case where $\left(1<R_{\rho}<2, R i<1\right)$, a low- $\epsilon$ mode is also apparent, with a population comparable to the high- $\epsilon$ mode. The documented trend is consistent with an association of the two modes with turbulence and salt fingering. In particular, we associate the low- $\epsilon$ mode, dominant at low $R_{\rho}$, with salt fingers. We associate the high $\epsilon$ mode, dominant at low $R i$, with turbulence.

Ruddick et al. (1997) used microstructure observations from a different instrument to examine dissipation at the NATRE site. The noise level of their measurements limited their analysis to observations having $\epsilon>7 \times 10^{-9} \mathrm{~W} \mathrm{~kg}^{-1}$. Their observations clearly fall into the high $\epsilon$ mode that we have associated with turbulence. Ruddick et al. (1997) use a Reynolds number, $R e=\epsilon /\left(\nu N^{2}\right)$, and they find no clear evidence of salt fingers in the parameter range $10^{1}<R e<10^{4}$. In a salt-finger regime, the parameter $\epsilon /\left(\nu N^{2}\right)$ is equivalent to the Stern number, $J_{b} /\left(\nu N^{2}\right)$. Stern (1969) argued that this parameter must be $O(1)$ for fingers to be active, while McDougall and Taylor (1984) found experimentally that the Stern number can be $O(10)$ for $R_{\rho}<2$. A characteristic Stern number for our low- $\epsilon$ mode is $\mathrm{O}(10)$. Thus, the Ruddick et al. (1997) Reynolds numbers are too large to admit the possibility for fingers. 
In examining only an upper threshold of our dissipation data, we have attempted to filter out weak dissipation events not likely associated with salt fingers or turbulence, as well as those sites where signals are weak relative to instrumental noise levels. Additionally, we will assume that subset of observations with $\epsilon\left(\chi>\chi_{75}\right)$ can be well modeled by the production-dissipation balances of (2.1) and (2.2). In doing so, we will expect the dissipation ratio analysis of this data to provide valuable information on the mixing processes to which the dissipation is attributable. By examining $\Gamma$ in the $\left(R_{\rho}, R i\right)$ parameter space, we may capitalize on the association between the two dissipative processes and their stability parameters. We expect $\Gamma$ to be consistent with the turbulence model at low $R i$ and with the salt-finger model at low (finger-favorable) values of $R_{\rho}$. There are regions of the $\left(R_{\rho}, R i\right)$ parameter space that do not favor either process. These include the large $R i$ region of the doubly stable regime, and the region of the finger-favorable regime where both $R i$ and $R_{\rho}$ are large. In these regions where the finger and shear instabilities are not favored, exceptional dissipative events should be rare.

\subsubsection{Statistical Treatment of Dissipation Data}

Mean and variance estimation of dissipation rate data has been discussed by many authors. The apparent tendency for the statistical distributions of $\chi$ and $\epsilon$ to be lognormal has produced arguments in favor of maximum likelihood estimation (MLE, Baker and Gibson 1987). The breakdown of lognormality assumptions has also been documented, and Davis (1996) concludes that arithmetic estimation is the most robust form of analysis. We have evaluated the different estimation methods through Monte Carlo exercises involving lognormally distributed random data. For random data distributions with characteristics reflecting those of our dissipation data, the discrepancy between MLE and arithmetic methods becomes less than $10 \%$ for as few as 200 degrees of freedom. For these reasons, arithmetic estimation was adopted as the analysis procedure for this study.

To compute estimates of $\Gamma\left(R_{\rho}, R i\right)$, ensemble averages were computed for all data within a discrete bin of $\left(R_{\rho}, R i\right)$ parameter space. The standard error of $\Gamma$ within the bin was computed as 

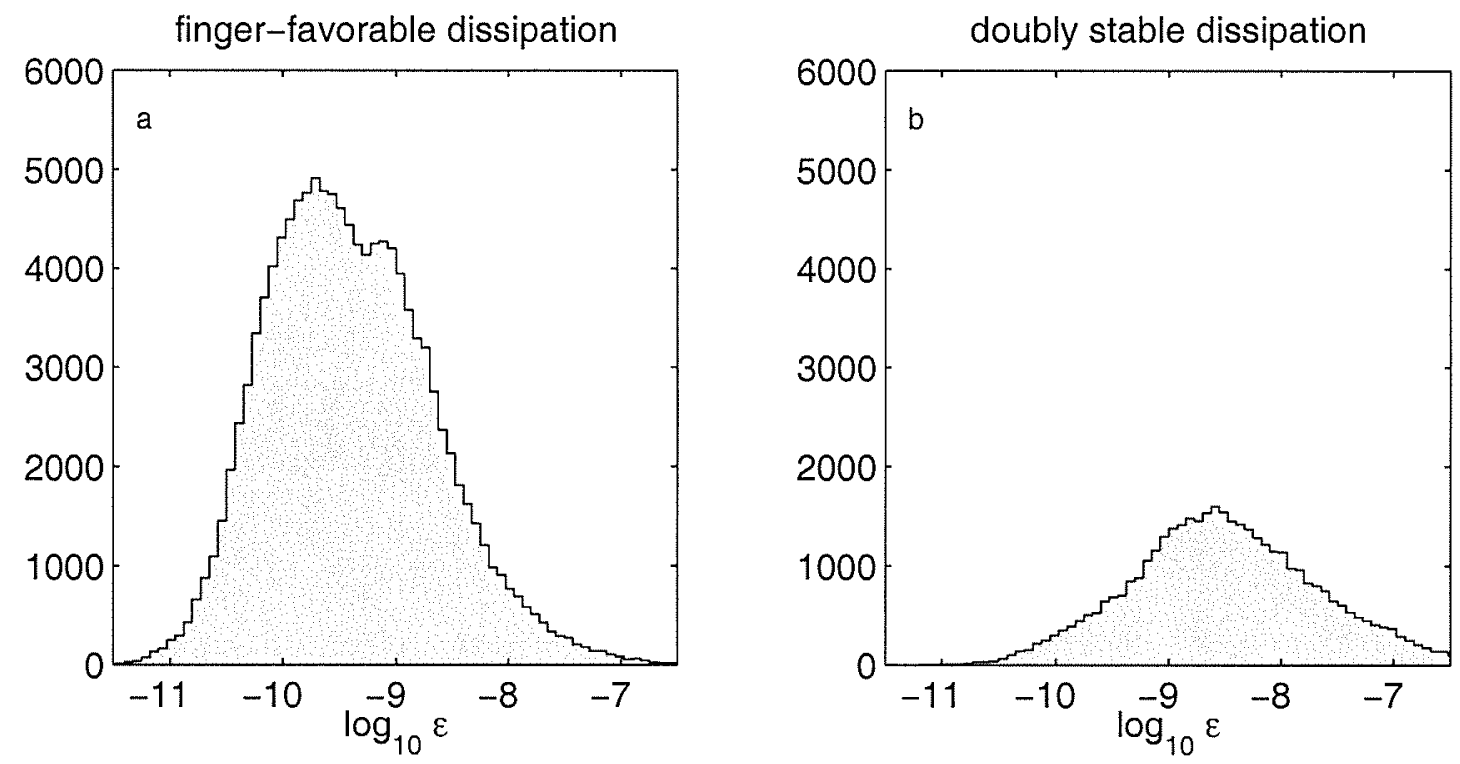

Figure 2.6: Histogram of the TKE dissipation rate for the subset of observations having $\chi>\chi_{75}$ for (a) all finger-favorable data and (b) all doubly stable data. The upper quartile of the combined TOPO and NATRE thermal dissipation record is $\chi \sim 1 \times 10^{-9} \mathrm{~K}^{-2} \mathrm{~s}^{-1}$. The bimodal structure in the finger-favorable histogram is statistically significant at the 0.15 level. The modes are located at $\epsilon \sim 2 \times$ $10^{-10} \mathrm{~W} \mathrm{~kg}^{-1}$ and $\epsilon \sim 1 \times 10^{-9} \mathrm{~W} \mathrm{~kg}^{-1}$. 

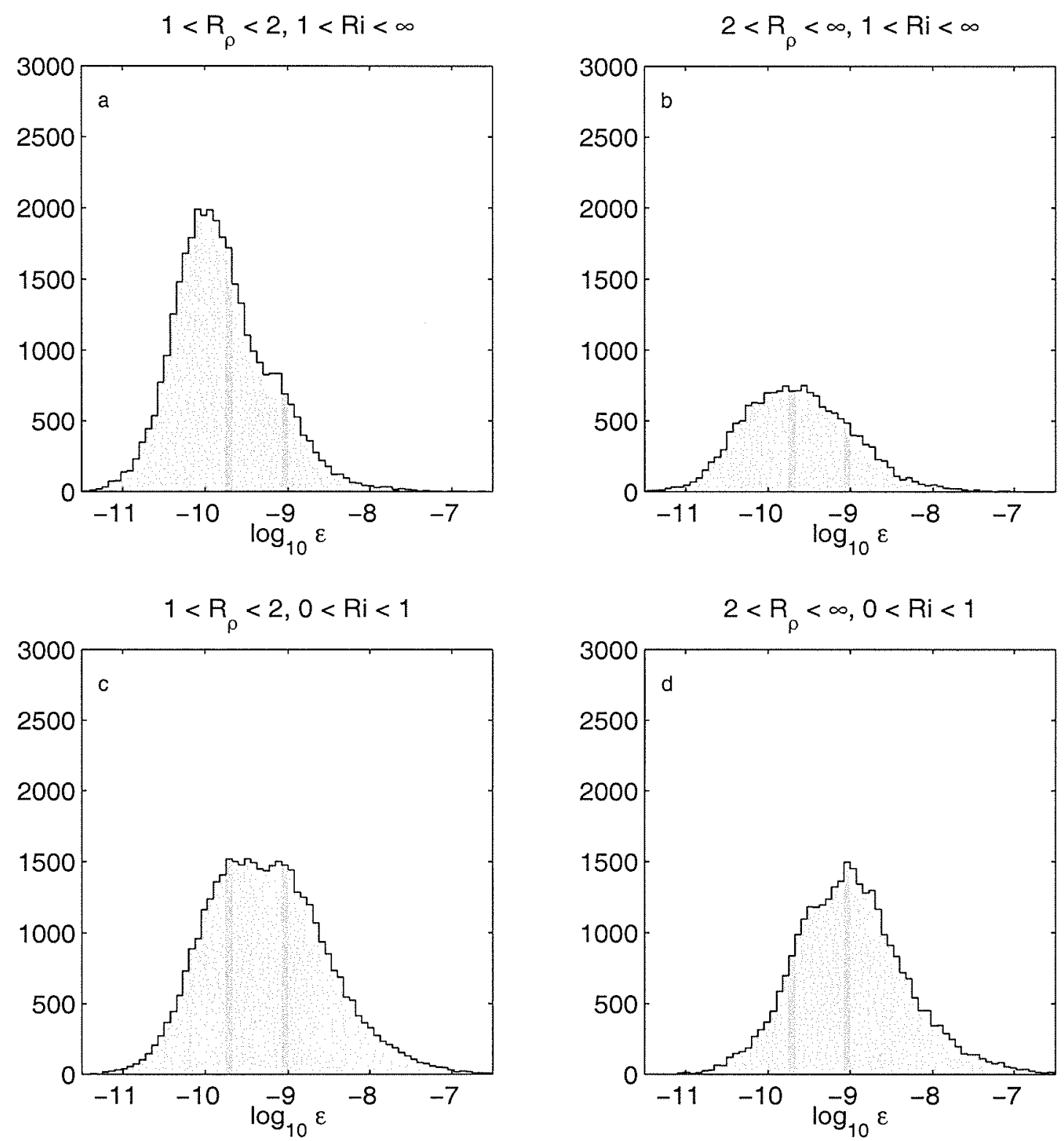

Figure 2.7: The finger-favorable distribution of $\epsilon\left(\chi>\chi_{75}\right)$ is broken into four distinct regions of $\left(R_{\rho}, R i\right)$ data space. In each histogram, the $\epsilon$ bins corresponding to the modes of the distribution shown in figure $2.6 \mathrm{a}$ are emphasized by darker shading. 


$$
\begin{aligned}
\delta \Gamma & =\frac{\Gamma}{\sqrt{n}}\left(\left(\frac{\delta \epsilon}{\langle\epsilon\rangle}\right)^{2}+\left(\frac{\delta \chi}{\langle\chi\rangle}\right)^{2}+\left(\frac{\delta N^{2}}{\left\langle N^{2}\right\rangle}\right)^{2}\right. \\
& \left.+\left(\frac{2 \delta \Theta_{z}}{\left\langle\Theta_{z}\right\rangle}\right)^{2}-\frac{2 \sigma^{2}}{\left\langle N^{2}\right\rangle\left\langle\Theta_{z}\right\rangle}\right)^{1 / 2},
\end{aligned}
$$

where the $\delta(\cdot)$ terms appearing in the parenthesis are the standard deviations, $\sigma^{2}$ is the covariance of $N^{2}$ and $\Theta_{z}^{2}$ and $n$ is the number of degrees of freedom of the data in the bin. The derivation of (2.8) follows standard error propagation methods (Bevington and Robinson 1992). Degrees of freedom in the 0.5-m $\epsilon$ and $\chi$ data were estimated using a vertical-lag correlation analysis. This was done for each of the data sets, with TOPO seamount summit data being treated separately from the seamount flank data. The NATRE dissipation profiles were characterized by correlation scales (vertical separation scales) of $5 \mathrm{~m}$ in the thermocline $(z<800 \mathrm{~m}$ ) and $10 \mathrm{~m}$ at greater depths. TOPO dissipation profiles were characterized by larger correlation scales, generally around $20 \mathrm{~m}$ for both seamount summit and flank profiles. A single degree of freedom is represented by the grouping of $0.5-\mathrm{m}$ data within one correlation scale in a single profile. For the ensemble of data in each bin, the number of such groupings gives the total degrees of freedom.

\subsubsection{Results}

We begin our examination of exceptional dissipation data within the doubly stable regime. The available data sets were subsampled in favor of all $0.5-\mathrm{m}$ dissipation observations with corresponding density ratio in the doubly stable range, $-100<$ $R_{\rho}<-1$. Data in the density ratio range between -1 and 0 were held from the analysis, as these data are characterized by weak thermal stratification where $\Theta_{z} \rightarrow 0$, making $\Gamma \propto N^{2} / \Theta_{z}^{2}$ singular. The data were assigned into a $\left(R_{\rho}, R i\right)$ data space by first selecting a bulk class of $R i$, and then subdividing the particular $R i$ population into bins of $R_{\rho}$. Bins of $R_{\rho}$ were each chosen to have 1000 elements of the $0.5-\mathrm{m}$ data. In this way, each $R_{\rho}$ bin generally contained 200-500 degrees of freedom. The mean and standard error of $\Gamma$ in each bin were then calculated using (2.5) and (2.8).

Analysis of the exceptional doubly stable dissipation data is shown in figure 2.8. The data were classified into six overlapping populations of $R i$. In each panel, the symbol and error bar denote the mean with $95 \%$ confidence interval for a 1000- 
element bin of data, with the symbol centered at the bin's mean density ratio. Richardson numbers less than 1 are considered in figures $2.8 \mathrm{a}, 2.8 \mathrm{~b}$ and $2.8 \mathrm{c}$. These data are characterized by $\Gamma$ between 0.1 and 0.25 , in good agreement with the results of previous studies (Moum 1996; Ruddick et al. 1997). Additionally, $\Gamma$ has no discernible density ratio dependence. We note that $75 \%$ of all exceptional dissipation data within the doubly stable regime occur when $R i<1$. The $25 \%$ of exceptional TOPO $\chi$ data that occur in patches where $R i>1$ are shown in figures 2.8d, $2.8 \mathrm{e}$ and $2.8 \mathrm{f}$. Data at $R i>5$ are too sparse to yield a single 1000 -element estimate. However, available data occurring at $R i>1$ show $\Gamma$ between 0.15 and 0.25 , numbers like those observed at lower values of the Richardson number.

Exceptional dissipation occurring in the finger-favorable regime was investigated by conditionally sampling the available data for patches with $R_{\rho}>1$. As was done for the doubly stable data, these data were conditionally sampled into six overlapping populations of $R i$ and then sorted by $R_{\rho}$ in bins of 1000 elements. Data from the NATRE survey contributes most of the finger favorable observations, with bins containing 400-700 degrees of freedom.

For finger-favorable observations with $R i<1$ (figures $2.9 \mathrm{a}, 2.9 \mathrm{~b}$ and $2.9 \mathrm{c}$ ), $\Gamma$ occupies the range of values exhibited by the doubly stable data, $0.1<\Gamma<0.3$. However, there is an elevation of $\Gamma$ at larger values of the Richardson number, consistent with expectations of salt fingers. To assess the significance of the observed trend in $\Gamma$ at large $R i$ within the context of salt-finger mixing, we can use (2.7) to give an expression for $r$ in terms of $\Gamma$,

$$
r=\frac{R_{\rho} \Gamma^{(f)}}{R_{\rho} \Gamma^{(f)}+R_{\rho}-1} .
$$

Using this relation, we have compared the $R i>5$ NATRE data (figure 2.9f) with the results of laboratory salt-finger observations, theoretical relations, and a numerical simulation (Shen, 1993). This comparison is shown in figure 2.10. Only the McDougall and Taylor (1984) experiments consistently achieved the low density ratio range most relevant for comparison with the NATRE data. The NATRE data dictate a buoyancy-flux ratio in the range $0.6<r<0.7$ for density ratios less than 1.6. Lower flux-ratio values of $0.4<r<0.5$ are inferred for $R_{\rho} \sim 2$. The range of $r$ values exhibited by the NATRE data is consistent with the laboratory data, noting that the $r$ values reported at $R_{\rho}=1.75$ by Schmitt 
(1979b) and Taylor and Bucens (1989) are the summaries for measurements made at density ratios as low as $R_{\rho}=1.6$. In particular, the NATRE data suggest a decline in flux ratio, also observed by McDougall and Taylor, as $R_{\rho}$ goes from 1 to 2 .

A formal statistical examination of the dissipation ratio signal was done after assembling a gridded $\left(R_{\rho}, R i\right)$ data space for both doubly stable and fingerfavorable regimes. As in the analysis described above, we have selected data from the upper quartile $\chi$ population of the combined TOPO and NATRE data set. This data space consisted of discrete data bins uniformly spaced in the set of transformed coordinates $\left(\log \left(\left|R_{\rho}\right|-1\right), \log (R i)\right)$. This choice of coordinates is favorable because it maps a wide range of parameter values, while emphasizing the parameter range $\left(R_{\rho}<2, R i<1\right)$ where most of the data lies. Figure 2.11 shows contour maps of $\Gamma$ for both stratification regimes. The shaded bins in each map contain 100 or more degrees of freedom. Bins with fewer degrees of freedom were excluded from the analysis. We find that the doubly stable regime is well characterized by a constant value of the dissipation ratio of $\Gamma=0.16 \pm 0.04$. In contrast, while much of the finger regime is characterized by $0.2<\Gamma<0.3$, elevated values of $\Gamma$ dominate the upper left (small $R_{\rho}$, large $R i$ ) quadrant of the map.

To evaluate the significance of the finger-regime results, we have implemented a simple statistical test. The dissipation ratio result from the doubly stable regime is taken as indicative of turbulent mixing. Specifically, we take $\Gamma^{(t)}=0.16 \pm$ 0.04 as the null hypothesis of mixing attributable to turbulence. We have tested the finger-regime results against this null hypothesis, seeking evidence that the dissipation ratio estimates of the finger-favorable data are different from those of the doubly stable data. The results of the two-tailed hypothesis test are shown in figure 2.12a. The standardized variable $Z=\left(\Gamma-\Gamma^{(t)}\right)\left(\delta \Gamma^{2}+\delta \Gamma^{(t)^{2}}\right)^{-1 / 2}$ was tested at the 0.01 significance level. The null hypothesis of $\Gamma=\Gamma^{(t)}$ is accepted in nearly all of quadrants I, III and IV of the finger-favorable regime. However, the data in quadrant II $\left(R_{\rho}<2, R i>1\right)$ strongly supports the alternate hypothesis $\Gamma \neq \Gamma^{(t)}$. A second statistical test was used to evaluate the possibility of salt-finger mixing. The finger model was evaluated using the laboratory $r$ data (figure 2.5b) and the null hypothesis of $\Gamma=\Gamma^{(f)}$ was tested at the 0.01 significance level using the test variable $Z=\left(\Gamma-\Gamma^{(f)}\right)\left(\delta \Gamma^{2}+\delta \Gamma^{(f)^{2}}\right)^{-1 / 2}$. Subject to this test, all of the data in 

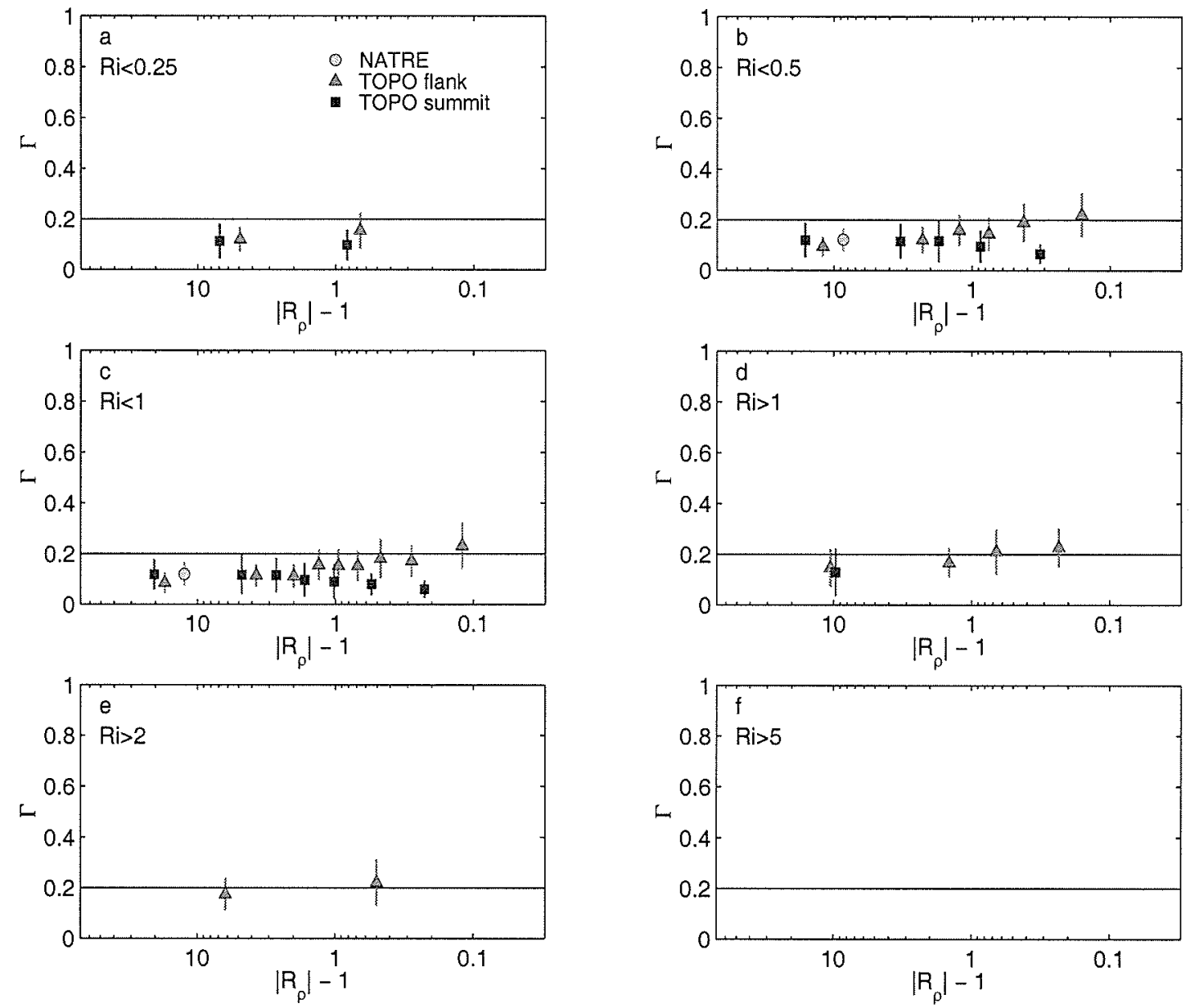

Figure 2.8: The dissipation ratio of doubly stable observations with $\chi>\chi_{75}$. The data were grouped into six different $R i$ populations (a-f). Estimates were derived separately for each of the $3 \mathrm{HRP}$ data groups: NATRE (circles), TOPO seamount flank (triangles) and TOPO seamount summit (squares). Each symbol is the estimate $\Gamma=\left(<\chi><N^{2}>\right)\left(2<\epsilon><\Theta_{z}>^{2}\right)^{-1}$, where the ensemble average was computed for a bin of 1000 dissipation estimates. The crror bars give the $95 \%$ confidence interval for a reduced number $(<1000)$ of degrees of freedom. In each panel, a reference value of $\Gamma=0.2$ is shown. 

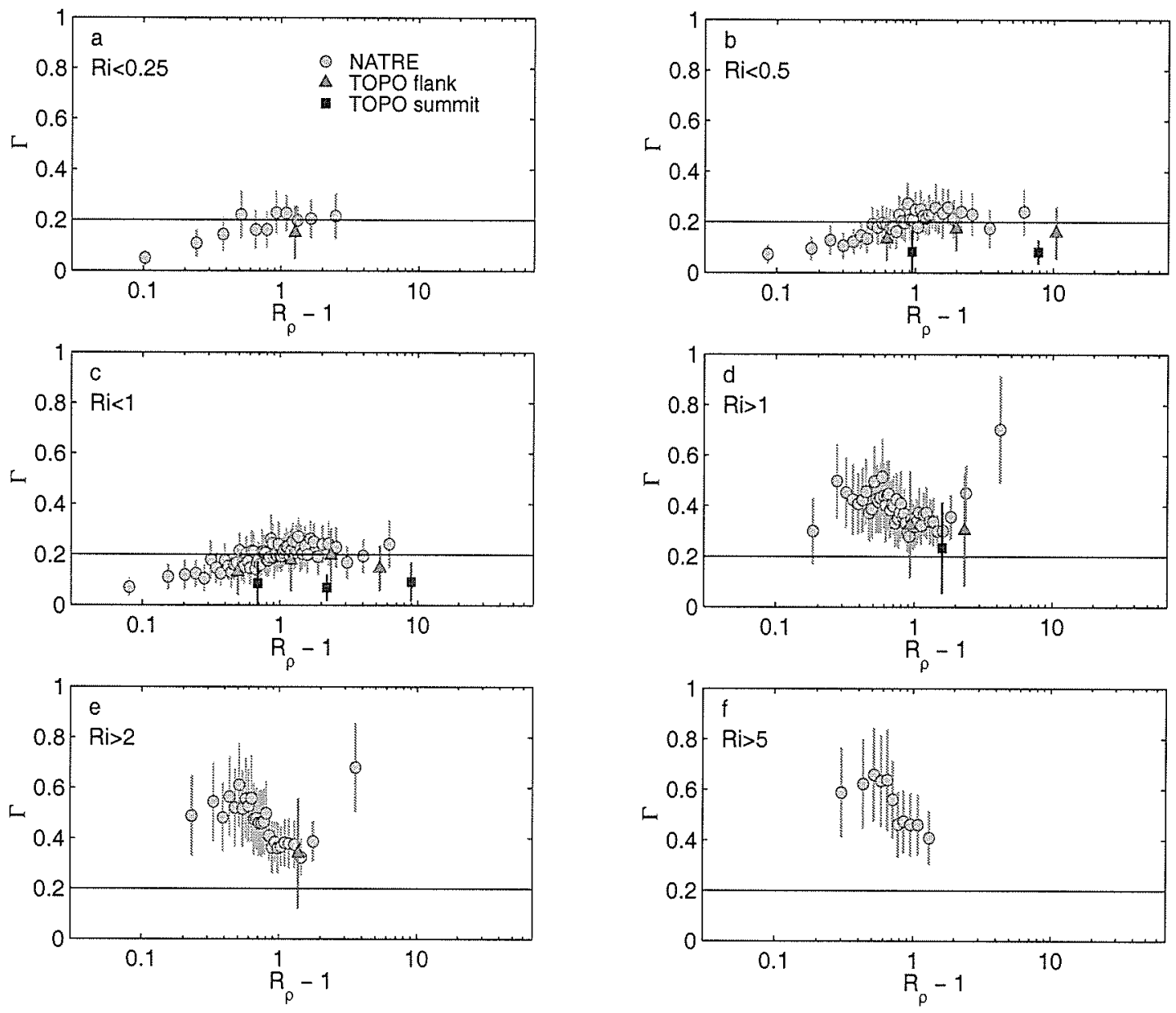

Figure 2.9: The dissipation ratio of finger-favorable observations with $\chi>\chi_{75}$. 


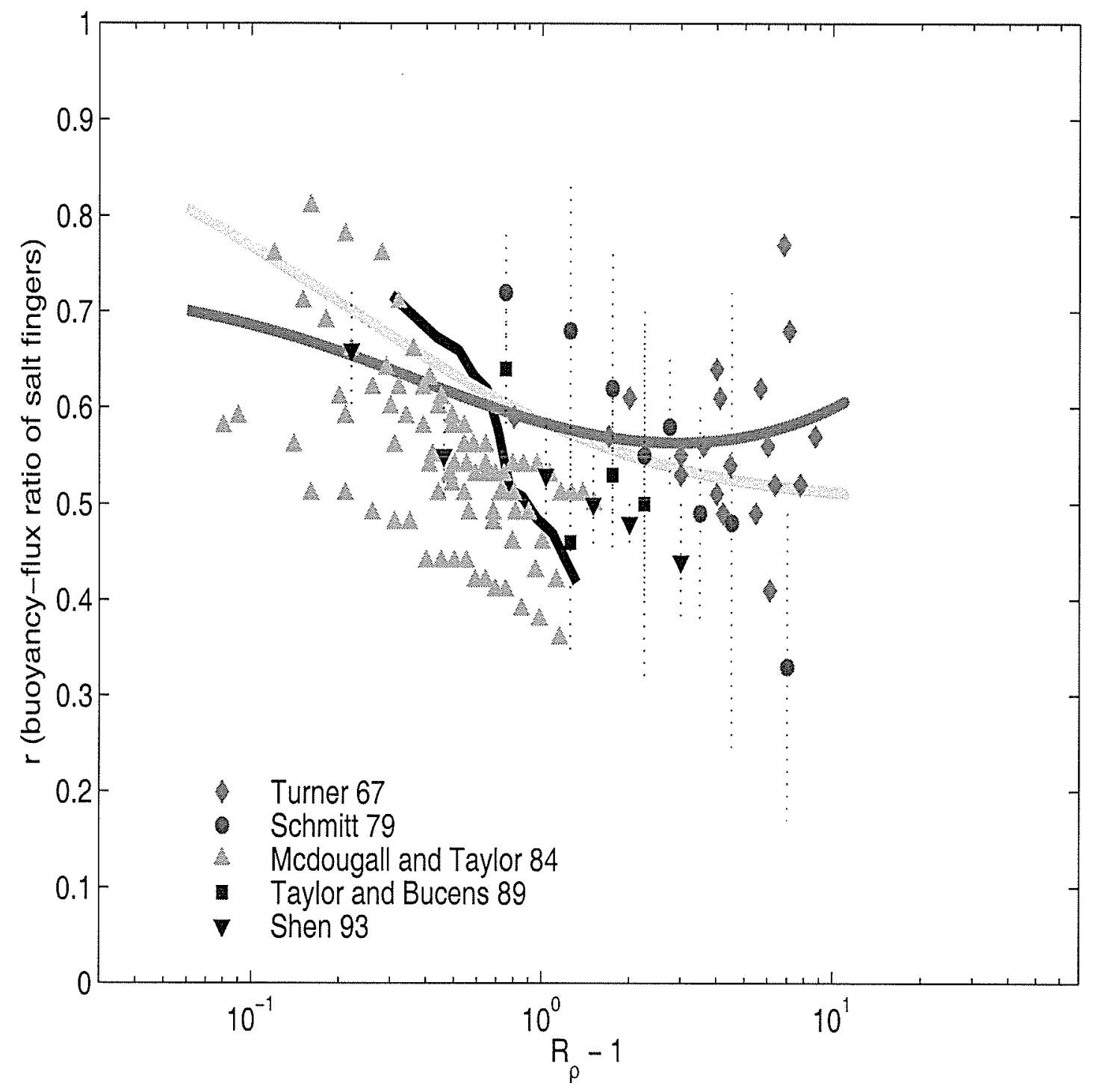

Figure 2.10: Estimates of the salt finger buoyancy-flux ratio $r=$ $\left(\alpha k_{\theta} \Theta_{z}\right)\left(\beta k_{s} S_{z}\right)^{-1}$. The results of four laboratory experiments are shown: Turner (1969, diamonds), Schmitt (1979b, circles), McDougall and Taylor (1984, upward triangles) and Taylor and Bucens (1989, squares). Also shown are the numerical results of Shen (1993, downward triangles) and the theoretical models of Stern (1975, light curve) and Schmitt (1979a, dark curve). Buoyancy-flux ratio estimates were derived from HRP observations by assuming that the NATRE $\left(\chi>\chi_{75}, R i>5\right)$ dissipation ratio signal (figure 2.9f) was entirely attributable to salt fingers. The relation $r=R_{\rho} \Gamma\left(R_{\rho} \Gamma+R_{\rho}-1\right)^{-1}$ was used. The HRP data-derived estimates are shown with a $99 \%$ confidence band. 


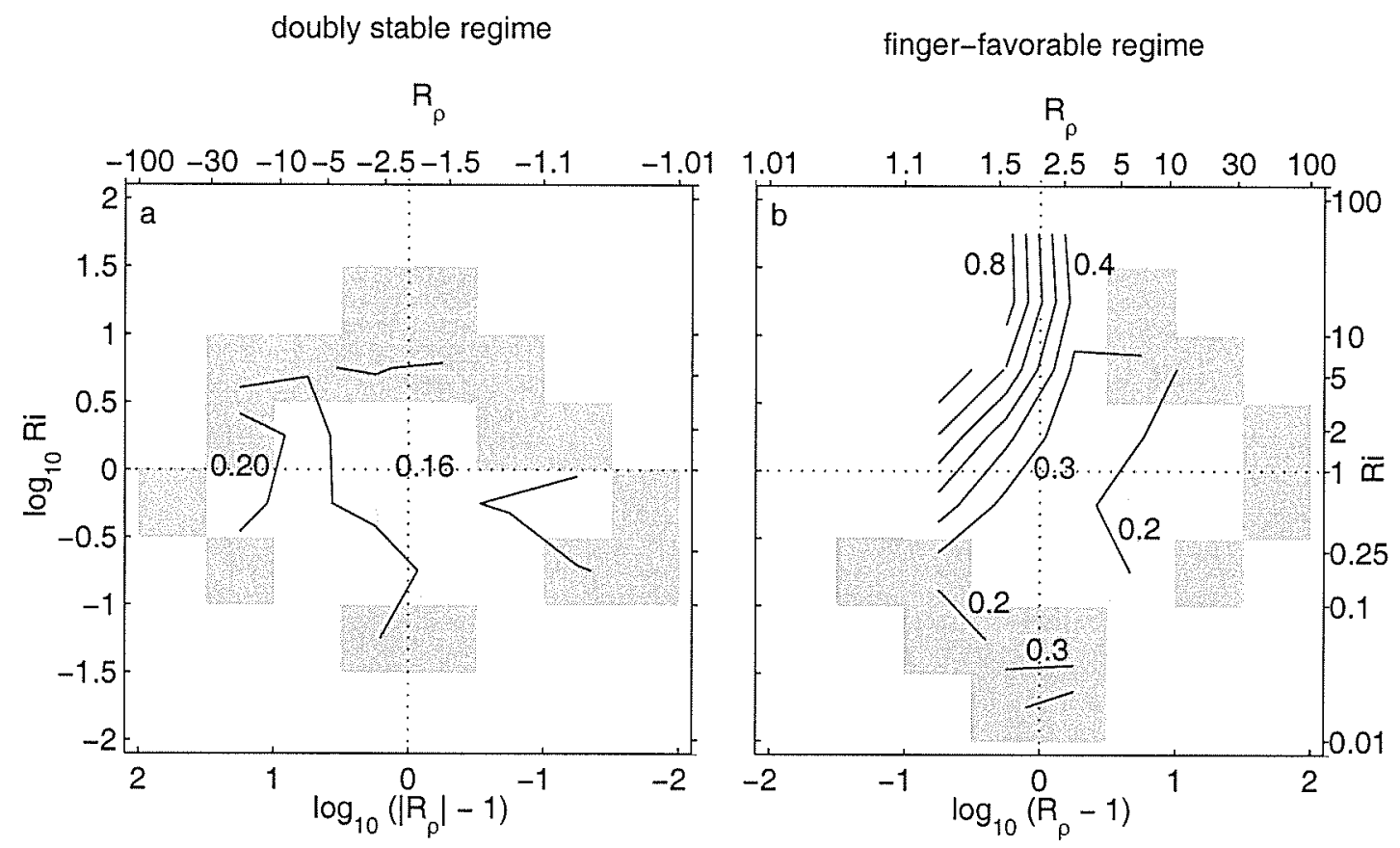

Figure 2.11: A contrast between $\Gamma\left(R_{\rho}, R i\right)$ of doubly stable and finger-favorable exceptional $\chi$ observations. Observations were grouped into bins of the $\left(\log \left(\left|R_{\rho}\right|-\right.\right.$ 1), $\log (R i))$ parameter space, and $\Gamma$ was estimated in bins where the data density exceeded 100 degrees of freedom (all shaded regions). In each panel, the estimate $\Gamma=\left(<\chi><N^{2}>\right)\left(2<\epsilon><\Theta_{z}>^{2}\right)^{-1}$ is contoured. Regions of darker shading denote bins where the uncertainty of $\Gamma$ exceeds $25 \%$ of the mean. The doubly stable regime (a) is characterized by a broad parameter range having $\Gamma=0.16$ (inner three contours). The finger-favorable regime (b) is characterized by larger values of $\Gamma$, particularly in the (low $R_{\rho}$, large $R i$ ) region of parameter space (quadrant II, $\left(R_{\rho}<2, R i>1\right)$ ). 


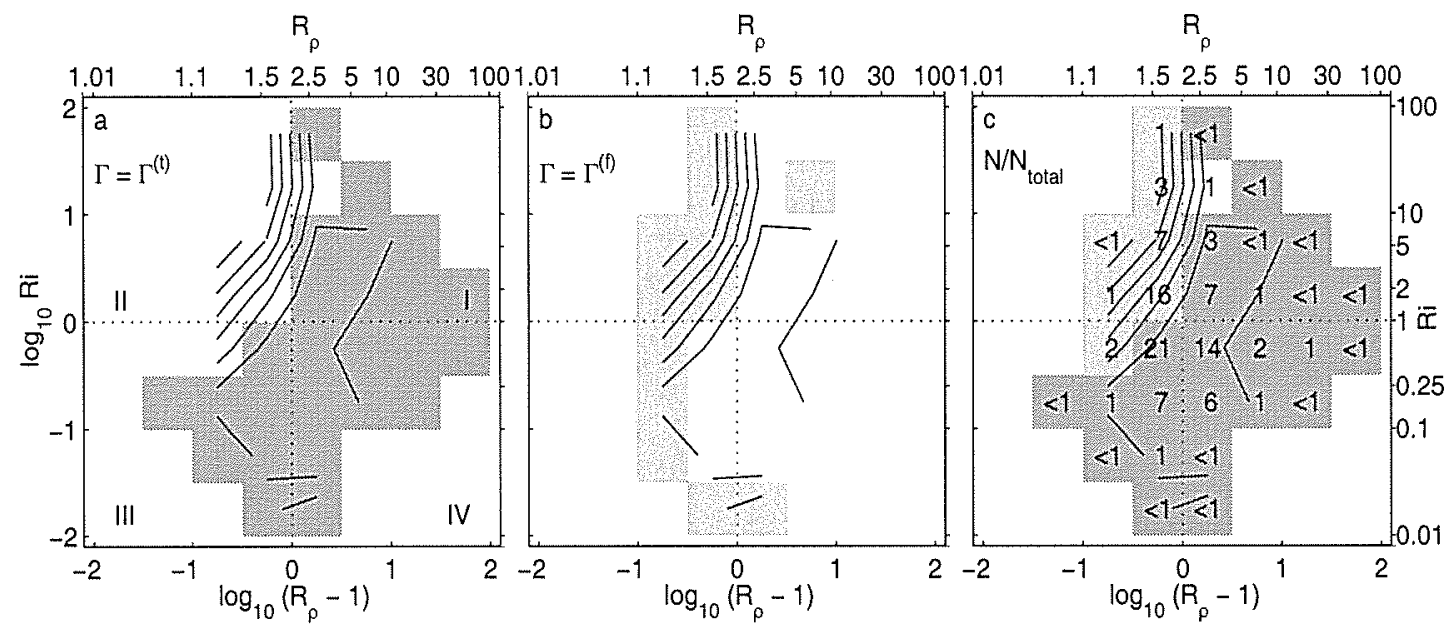

Figure 2.12: Statistical classification of the exceptional $\chi$ observations in the finger-favorable regime. (a) The observations were tested against the null hypothesis that $\Gamma$ was attributable to turbulence. The finger-favorable regime can be broken into the four quadrants defined by the $\left(\log \left(R_{\rho}-1\right), \log (R i)\right)$ data space (I-IV as labeled). Most of quadrants I, III and IV are consistent with the turbulence hypothesis at the 0.01 significance level (99\% confidence), and these bins are identified by dark shading. (b) The observations were tested against the null hypothesis that $\Gamma$ was attributable to salt fingers. Data consistent with the finger hypothesis are indicated by dark shading. All of the quadrant II data meet consistency with the finger model at the 0.01 significance level, as do several bins already found to be consistent with turbulence. (c) The percentage of total data represented by each bin is reported. Those data with degenerate classification are assigned to turbulence. This results in the classification of nearly $70 \%$ of the data as turbulence (darkest shading) and $30 \%$ as salt fingers (medium shading). $N_{\text {total }}=119,570$ 
quadrant II meets consistency with the finger model (figure 2.12b). As is apparent after comparing figures $2.12 \mathrm{a}$ and $2.12 \mathrm{~b}$, a small class of data is consistent with both models for the dissipation ratio. This degenerate class of data is the result of: (i) the overlap of the $\Gamma^{(t)}$ and $\Gamma^{(f)}$ models when $R_{\rho}<1.4$ and (ii) bins having large values of $\delta \Gamma$. In classifying this degenerate data, we have given preference to the turbulence model. Thus, we attribute the dissipation ratio signal to turbulence in nearly all of quadrants I, III and IV, while quadrant II is associated with fingers. This resulting classification is shown in figure 2.12c along with the percentage of observations represented in each bin. Through the application of these two tests, we have classified all but $1 \%$ of our $\chi>1 \times 10^{-9} \mathrm{~K}^{2} \mathrm{~s}^{-1}$ data as either attributable to turbulence or salt fingers.

By testing the original null hypothesis of turbulence at the 0.01 significance level, we have allowed for very generous uncertainty in the value of $\Gamma^{(t)}$. This also makes the rejection of the null hypothesis very difficult. In this manner we have been very conservative in labeling any binned $\chi>\chi_{75}$ data as "nonturbulence". Similarly, by testing the "nonturbulent" observations against a salt-finger hypothesis at the 0.01 significance level, we have allowed generous uncertainty in the $\Gamma^{(f)}$ model. For these reasons, we view this work as a consistency check. The upper quartile of $\chi$ observations having stability parameters in the range $\left(R_{\rho}<2, R i>1\right)$ can be attributed to salt fingers in a manner consistent with available information from theory and laboratory work.

\subsection{Mixing at the NATRE Site}

\subsubsection{Vertical Mixing Influenced by Salt Fingers}

We seek estimates of the net diffusivities $k_{\theta}$ and $k_{s}$ that dictate the vertical fluxes over $\mathrm{O}(100 \mathrm{~m})$ scales. In such estimates, the presence of salt-finger mixing will tend to enhance the haline diffusivity over the thermal diffusivity. Since there is a high occurrence of patches with low $R_{\rho}$ and stable $R i$ at the level of the tracer injection in NATRE, we find much of the dissipation can be ascribed to salt fingers.

To derive estimates of the net diffusivities, we employ 100 uniformly spaced 
stations from a $400 \mathrm{~km} \times 400 \mathrm{~km}$ survey grid (figure 2.1). This April 1992 survey took 18 days to complete. Each profile includes a full record of dissipation data to $2000 \mathrm{~m}$. Our goal is to estimate survey-scale diffusivities applicable to the synoptic snapshot represented by our survey. We make the assumption that the variance budgets (1) and (2) are valid within the context of: (i) survey scale lateral averaging, (ii) $\mathrm{O}(100 \mathrm{~m}$ ) vertical averaging, and (iii) the time average invoked by treating the 18 day survey as a snapshot.

The mean field was derived with reference to a neutral-density coordinate system $\left(\sigma_{n}\right.$, Jackett and McDougall 1997). Epineutral (i.e., along neutral surface) averaging yielded mean profiles of $\bar{\Theta}$ and $\bar{S}$ in terms of a vertical coordinate $\bar{z}$, the mean depth of each density surface. All mean field variables are associated with some uncertainty. In the case of $\bar{\Theta}$ and $\bar{S}$, standard errors as large as $\delta \bar{\Theta}=0.02 \mathrm{~K}$ and $\delta \bar{S}=0.005$ (values typical of the $100 \mathrm{~m}$ deep neutral surface) can be ascribed to mesoscale eddies. The uncertainty in the mean vertical coordinate $\bar{z}$ is more limiting, with $\delta \bar{z}=5 \mathrm{~m}$. The variability associated with $\bar{z}$ is attributable to large-amplitude internal tides. Mean vertical gradient quantities $\left(\overline{\Theta_{z}}, \overline{S_{z}}, \overline{N^{2}}\right)$ were calculated from $\mathrm{O}(100 \mathrm{~m})$ linear fits to $\bar{\Theta}(\bar{z})$ and $\bar{S}(\bar{z})$. The dominant uncertainty in the vertical gradients comes from $\delta \bar{z}$, yielding a $2 \%$ error in the $\overline{N^{2}}$ estimates.

The vertical diffusivities $k_{\theta}$ and $k_{s}$ were calculated using a scheme that follows from the results of our $\left(R_{\rho}, R i\right)$ analysis of $\Gamma$. The general form of the model we will employ has similarities to the model suggested by McDougall and Ruddick (1992). The differences between our approach and that of McDougall and Ruddick (1992) will be described in section 2.5. Our approach is motivated by the results of the dissipation ratio analysis of exceptional $\chi$ data (section 2.3.5). Within each layer, all dissipation data are partitioned into two groups. All 0.5-m $\epsilon$ and $\chi$ estimates associated with $\left(1<R_{\rho}<2, R i>1\right)$ are presumed salt-finger dissipation. All dissipation associated with $R_{\rho}$ and $R i$ outside the finger-favorable range (i.e., the compliment of $\left.\left(1<R_{\rho}<2, R i>1\right)\right)$ are attributed to turbulence. In this manner, we attribute some percentage of the dissipation observations to fingers $\left(P^{(f)}\right)$ and turbulence $\left(P^{(t)}=1-P^{(f)}\right)$. In using this approach, we assume that the regime partition found for exceptional $\chi$ is more generally applicable to the ensemble averages of dissipation profiles. We will further discuss the applicability of this model in section 2.5 . 


\begin{tabular}{ccccccr}
\hline $\begin{array}{c}\bar{z} \\
(\mathrm{~m})\end{array}$ & $\overline{R_{\rho}}$ & $P^{(f)}$ & $P^{(t)}$ & $\begin{array}{c}k_{\theta} \\
\left(\mathrm{cm}^{2} \mathrm{~s}^{-1}\right)\end{array}$ & $\begin{array}{c}k_{s} \\
\left(\mathrm{~cm}^{2} \mathrm{~s}^{-1}\right)\end{array}$ & $\begin{array}{c}k_{\rho} \\
\left(\mathrm{cm}^{2} \mathrm{~s}^{-1}\right)\end{array}$ \\
\hline \hline 90 & 1.63 & 0.10 & 0.90 & $0.93 \pm 0.19$ & $1.22 \pm 0.25$ & $0.48 \pm 0.63$ \\
190 & 1.65 & 0.44 & 0.56 & $0.12 \pm 0.02$ & $0.22 \pm 0.03$ & $-0.03 \pm 0.06$ \\
300 & 1.71 & 0.48 & 0.52 & $0.08 \pm 0.01$ & $0.13 \pm 0.01$ & $0.01 \pm 0.03$ \\
400 & 1.91 & 0.35 & 0.65 & $0.07 \pm 0.01$ & $0.10 \pm 0.01$ & $0.03 \pm 0.03$ \\
500 & 2.16 & 0.09 & 0.91 & $0.07 \pm 0.01$ & $0.08 \pm 0.01$ & $0.06 \pm 0.03$ \\
600 & 2.46 & 0.05 & 0.95 & $0.06 \pm 0.01$ & $0.07 \pm 0.01$ & $0.05 \pm 0.02$ \\
700 & 2.53 & 0.08 & 0.92 & $0.06 \pm 0.01$ & $0.09 \pm 0.01$ & $0.04 \pm 0.02$ \\
800 & 2.57 & 0.10 & 0.90 & $0.07 \pm 0.01$ & $0.12 \pm 0.02$ & $0.04 \pm 0.02$ \\
\hline
\end{tabular}

Table 2.1: Net diffusivity estimates.

Diffusivities in each layer are first calculated for the two dissipative regimes. In the case of turbulent dissipation, we use the Osborn (1980) diffusivity model,

$$
k_{0}^{(t)}=\Gamma^{(t)}<\epsilon>^{(t)}\left(\overline{N^{2}}\right)^{-1},
$$

where $\langle\epsilon\rangle^{(t)}$ denotes the ensemble average of the $\epsilon$ identified as attributable to turbulence, and $\Gamma^{(t)}$ is the dissipation ratio for turbulence. We take $k_{0}^{(t)}$ as the diffusivity for both heat and salt. In these calculations, we use $\Gamma^{(t)}=0.2$, the value consistent with most previous work. The standard error of the turbulence diffusivity is also calculated,

$$
\delta k_{0}^{(t)}=k_{0}^{(t)} \cdot\left(\left(\frac{\delta \Gamma^{(t)}}{\Gamma^{(t)}}\right)+\left(\frac{\delta<\epsilon>^{(t)}}{<\epsilon>^{(t)}}\right)+\left(\frac{\delta \overline{N^{2}}}{\overline{N^{2}}}\right)\right)^{1 / 2}
$$

We have calculated $\delta\langle\epsilon\rangle^{(t)}$ using a bootstrap method (Efron and Gong 1983). The number of degrees of freedom in the bootstrap estimates were determined from the number of nonoverlapping 5 -m profile segments in the ensemble. For the uncertainty of the turbulent dissipation ratio, we have used the result of our doubly stable data analysis, $\delta \Gamma^{(t)}=0.04$ (figure 2.11a). In the case of salt-finger dissipation, we use the Osborn and Cox (1972) diffusivity model,

$$
k_{\theta}^{(f)}=0.5<\chi>^{(f)}\left({\overline{\Theta_{z}}}^{2}\right)^{-1},
$$

where $\langle\chi\rangle^{(f)}$ denotes the ensemble average of the $\chi$ observations identified 
with salt fingers. The haline diffusivity is then calculated through use of the buoyancy-flux ratio of fingers, $r=\left(\alpha k_{\theta}^{(f)} \overline{\Theta_{z}}\right)\left(\beta k_{s}^{(f)} \overline{S_{z}}\right)^{-1}$. Thus,

$$
k_{s}^{(f)}=\frac{\overline{R_{\rho}}}{r} \cdot k_{\theta}^{(f)} .
$$

where $\overline{R_{p}}$ is the density ratio computed from the $\mathrm{O}(100 \mathrm{~m})$ scale vertical gradients. (We note that $\overline{R_{\rho}}$ is not necessarily equal to the ensemble average of localized $5-\mathrm{m}$ $R_{\rho}$ estimates.) In (2.13) we use a buoyancy-flux ratio of $r=0.6 \pm 0.04$, a value taken from the $1.5<R_{\rho}<2$ laboratory data (figure 2.10). The net diffusivities for each layer are then calculated as the weighted average of the regime-based diffusivities,

$$
\begin{aligned}
& k_{\theta}=P^{(t)} \cdot k_{0}^{(t)}+P^{(f)} \cdot k_{\theta}^{(f)}, \\
& k_{s}=P^{(t)} \cdot k_{0}^{(t)}+P^{(f)} \cdot k_{s}^{(f)} .
\end{aligned}
$$

Thus, the net diffusivities depend on both the frequency and magnitude of the salt-finger and turbulent dissipation events. For a profile with no finger-favorable mixing $\left(P^{(f)}=0\right)$, both diffusivities converge to the value $k_{0}^{(t)}$.

Estimates of net diffusivities for each layer, as well as the weighting factors $P^{(f)}$ and $P^{(t)}$, are given in table 2.1. Figure 2.13 presents a summary of these results. Density ratio information is given in figure 2.13a. For each layer, the median value of the $5-\mathrm{m} R_{\rho}$ is shown as a vertical line. The density ratio range encompassed by the lower and upper quartile is also shown, as indicated by the shaded band within each layer. The density ratio calculated for the $100-\mathrm{m}$ scale density ratio $\left(\overline{R_{\rho}}\right)$ is also shown. We note that $\overline{R_{\rho}}$ is generally close to the median 5 -m $R_{\rho}$ in layers where intrusive features are infrequent. The near-surface layer contains the largest variability of 5-m $R_{\rho}$, with intrusive features also occurring at $\bar{z}>600 \mathrm{~m}$. These later features are associated with the upper boundary of the Mediterraneanproperty tongue. Figure 2.13b shows estimates of the diffusivities derived from (2.14). The haline diffusivity is enhanced over the thermal diffusivity in the upper thermocline, $150 \mathrm{~m}<\bar{z}<450 \mathrm{~m}$, where $0.5-\mathrm{m}$ scale patches are characterized by $R_{\rho}<2$. Near $\bar{z}=500 \mathrm{~m}$, the two diffusivities converge as $R_{\rho} \geq 2$. However, beneath $\bar{z}=600 \mathrm{~m}$, low- $R_{\rho}$ patches attributable to intrusive features are adequate to enhance $k_{s}$ over $k_{\theta}$ even though the large-scale stratification is characterized by $\overline{R_{\rho}} \approx 2.5$. Also shown are the estimates of the tracer diffusivity reported by 


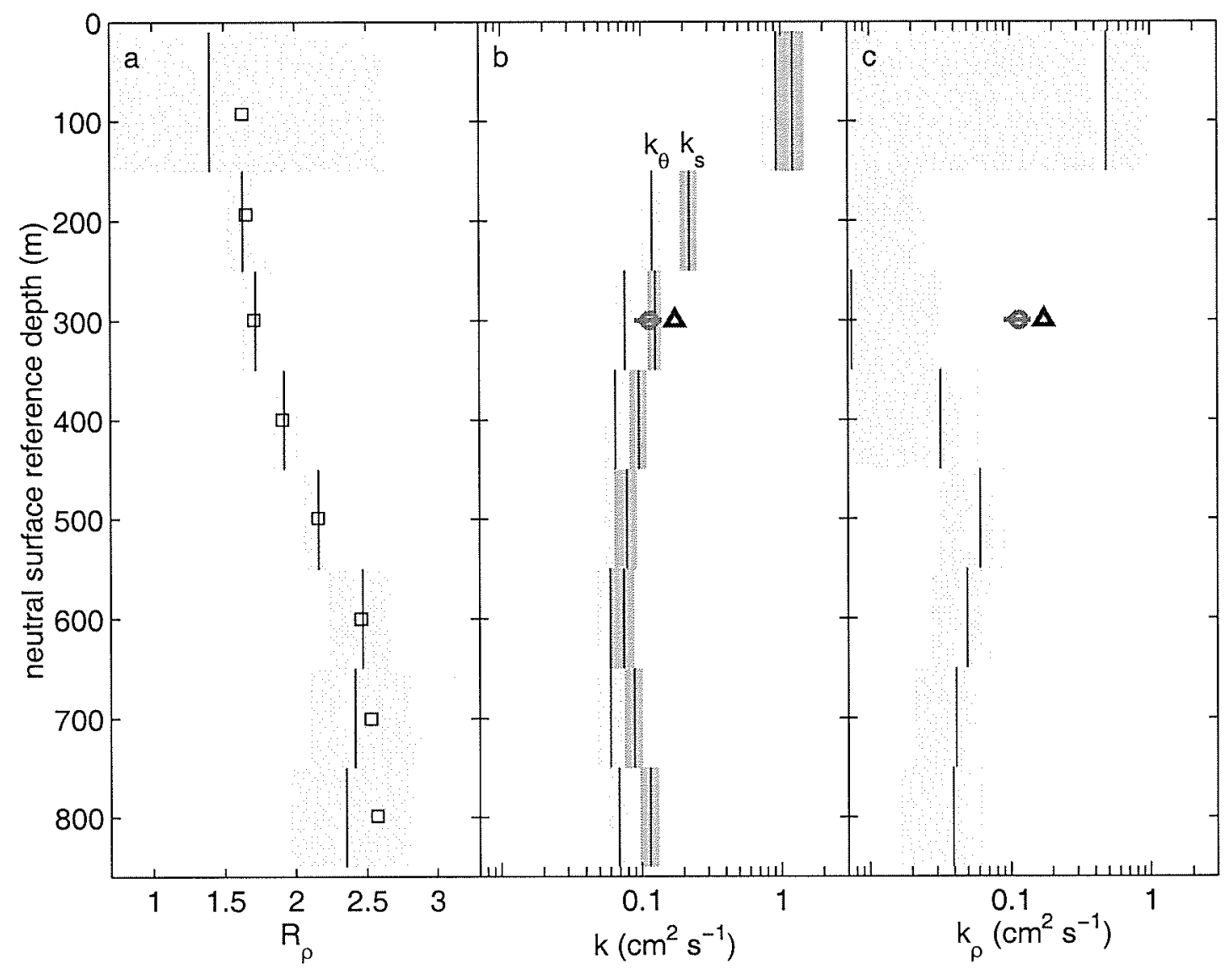

Figure 2.13: Results from the analysis of neutral density layers at the NATRE site. The mean depth of the neutral surfaces are used as the reference vertical coordinate. (a) The density ratio structure of each layer is shown. The shaded box is the interquartile range of the $5-\mathrm{m} R_{\rho}$, with the median value shown as the vertical line. The large-scale density ratio is computed from the layer-averaged $\overline{\Theta_{z}}$ and $\overline{S_{z}}$, and this value is shown in each box by the open square. (b) Profiles of the thermal $\left(k_{\theta}\right)$ and haline $\left(k_{s}\right)$ vertical diffusivities. The tracer-derived diffusivities (Ledwell et al. 1998) at the $\bar{z}=300 \mathrm{~m}$ surface are also shown. The circle gives the tracer-derived estimate for May through November 1992, the period closest in time to the HRP survey. The triangle gives the average tracer diffusivity over the later 2 years of the experiment (November 1992 through November 1994). If the vertical diffusivities of heat and salt differ, the tracer diffusivity should be that of salt. (c) The diffusivity of density derived from $k_{\theta}$ and $k_{s}$ using $k_{\rho} \overline{N^{2}}=g\left(\alpha k_{\theta} \overline{\Theta_{z}}-\beta k_{s} \overline{S_{z}}\right)$. The relative sizes of error bars in (b) and (c) is skewed by the logarithmic x-axis. 
Ledwell et al. (1998). The tracer was released at the $\sigma_{\theta}=26.75 \mathrm{~kg} \mathrm{~m}^{-3}$ isopycnal, close to the $\bar{z}=300 \mathrm{~m}$ neutral surface used in our analysis. The eddy diffusivity of the chemical tracer (sulfur hexafluoride) is expected to be equal to that of salt. Our estimate of $k_{s}$ agrees best with the tracer estimate derived for May 1992 through November 1992, the period immediately following the HRP survey. Figure $2.13 \mathrm{c}$ shows the diffusivity of density $\left(k_{\rho}\right)$ inferred from $k_{\theta}$ and $k_{s}$ using the relation

$$
k_{\rho} \overline{N^{2}}=g\left(\alpha\left(k_{\theta} \overline{\Theta_{z}}\right)-\beta\left(k_{s} \overline{S_{z}}\right)\right)
$$

In the upper $300 \mathrm{~m}, k_{\rho}$ is near zero and possibly negative. The diffusivity for density is less than $k_{\theta}$ and $k_{s}$ in all layers where $k_{s}>k_{\theta}$. Furthermore, $k_{\rho}$ clearly underestimates the tracer derived diffusivity. At $\bar{z}=500 \mathrm{~m}$, where fingers contribute little dissipation, the diffusivities are nearly equal.

\subsubsection{Estimates of Diapycnal Advection}

With estimates of the vertical diffusivities derived from (2.14), we may estimate not only the vertical fluxes of heat and salt, but also the vertical flux divergence of buoyancy. The flux divergence of buoyancy is of interest because it will dictate the strength of the advection between layers of different density. This diapycnal advection $\left(w_{*}\right)$ is strictly the diapycnal component of the total vertical velocity, $w_{(\text {total })}=\underline{u} \cdot \nabla z_{n}+w_{*}$, where $z_{n}$ is the interface between two neutral density layers (Pedlosky 1996). An equation for the diapycnal advection has been derived by McDougall (1991). Here we use a form of the McDougall (1991) expression suitable for vertical differentiation in a layered neutral density system with nonequal thermal and haline diffusivities,

$$
w_{*} \frac{g}{N^{2}}=\alpha \sigma_{z} \frac{\partial}{\partial \sigma}\left(k_{\theta} \overline{\Theta_{z}}\right)-\beta \sigma_{z} \frac{\partial}{\partial \sigma}\left(k_{s} \overline{S_{z}}\right)-\kappa\left(C_{b} \nabla \bar{\Theta} \cdot \nabla \bar{\Theta}+T_{b} \nabla \bar{\Theta} \cdot \nabla \bar{P}\right),
$$

where $\sigma$ and $\sigma_{z}$ are neutral density and its vertical derivative. In (2.16), $\kappa$ is the epineutral diffusivity (i.e., the diffusivity acting along neutral surfaces), $\nabla$ is the epineutral gradient operator, and $\bar{P}$ is the pressure along the neutral surface. Thus, the diapycnal velocity depends not only on the vertical-flux divergence 


\begin{tabular}{ccccc}
\hline $\begin{array}{c}z \\
(\mathrm{~m})\end{array}$ & $\begin{array}{c}\frac{g \alpha \sigma_{z}}{\overline{N^{2}}}\left(k_{\theta} \overline{\Theta_{z}}\right)_{\sigma} \\
\left(\mathrm{m} \mathrm{yr}^{-1}\right)\end{array}$ & $\begin{array}{c}\frac{-g \beta \sigma_{z}}{\bar{N}^{2}}\left(k_{s} \overline{S_{z}}\right)_{\sigma} \\
\left(\mathrm{m} \mathrm{yr}^{-1}\right)\end{array}$ & $\begin{array}{c}\frac{-g \kappa}{N^{2}} C_{b} \nabla \bar{\Theta}^{2} \\
\left(\mathrm{~m} \mathrm{yr}^{-1}\right)\end{array}$ & $\begin{array}{c}\frac{-g \kappa}{\overline{N^{2}}} T_{b} \nabla \bar{\Theta} \nabla \bar{P} \\
\left(\mathrm{~m} \mathrm{yr}^{-1}\right)\end{array}$ \\
\hline \hline 90 & & & $-0.48 \pm 0.24$ & $-0.02 \pm 0.01$ \\
190 & $23.24 \pm 5.19$ & $-20.18 \pm 4.65$ & $-0.10 \pm 0.05$ & $0.00 \pm 0.00$ \\
300 & $3.64 \pm 0.86$ & $-5.33 \pm 0.89$ & $-0.03 \pm 0.01$ & $0.01 \pm 0.00$ \\
400 & $1.24 \pm 0.60$ & $-2.26 \pm 0.38$ & $-0.02 \pm 0.01$ & $0.01 \pm 0.00$ \\
500 & $0.80 \pm 0.50$ & $-1.15 \pm 0.28$ & $-0.02 \pm 0.01$ & $0.01 \pm 0.00$ \\
600 & $0.43 \pm 0.51$ & $-0.24 \pm 0.24$ & $-0.01 \pm 0.01$ & $0.00 \pm 0.00$ \\
700 & $-0.18 \pm 0.43$ & $0.24 \pm 0.21$ & $-0.14 \pm 0.07$ & $-0.01 \pm 0.01$ \\
800 & & & $-0.54 \pm 0.27$ & $0.00 \pm 0.01$ \\
\hline
\end{tabular}

Table 2.2: Components of the dianeutral advection equation.

of density, but also on the lateral divergence of $\bar{\Theta}$ and $\bar{S}$ fluxes by mesoscale eddies. Since neutral surfaces are defined by the relation $\alpha \nabla \bar{\Theta}=\beta \nabla \bar{S}$, epineutral fluxes are divergent only through the nonlinear terms in the equation of state. In particular, the process of cabbeling is represented through the term $C_{b} \nabla \bar{\Theta} \cdot \nabla \bar{\Theta}$, with $C_{b} \sim \partial \alpha / \partial \Theta$. The second nonlinear thermodynamic process is the so-called thermobaric effect, represented by $T_{b} \nabla \bar{\Theta} \cdot \nabla \bar{P}$ with $T_{b} \sim \partial \alpha / \partial P$. We further note that (2.16) is the complete specification of the advection through a neutral surface. The equation is appropriate for fully three-dimensional flow.

The vertical-flux divergence contribution to $w_{*}$ was computed by differentiating the vertical fluxes of heat $\left(F_{\theta}=-k_{\theta} \overline{\Theta_{z}}\right)$ and salt $\left(F_{s}=-k_{s} \overline{S_{z}}\right)$ using a centraldifference scheme with an associated error propagation formula. The epineutral contributions to the diapycnal advection were also estimated. An epineutral diffusivity of $\kappa=1000 \pm 500 \mathrm{~m}^{2} \mathrm{~s}^{-1}$ was used in these calculations. This $\kappa$ is consistent with the value reported by Ledwell et al. (1998) for the lateral diffusivity acting along the tracer release surface. The epineutral gradients of $\bar{\Theta}$ and $\bar{P}$ were calculated from the hydrography of the NATRE HRP survey. The $\bar{\Theta}$ and $\bar{P}$ fields were mapped onto a quadratic plane of the form

$$
\Phi(X, Y)=\phi_{0}+\phi_{x} X+\phi_{y} Y+\phi_{x x} X^{2}+\phi_{x y} X Y+\phi_{y y} Y^{2},
$$

where $(\mathrm{X}, \mathrm{Y})$ are the Cartesian coordinates centered on the survey and $\phi_{(\cdot)}$ are the coefficients of the least-square fit. Degrees of freedom in the lateral scalar fields were determined by examining spatial-lag correlations. While all 100 stations 
are treated as independent for the $\bar{\Theta}$ calculations, the $\bar{P}$ field contained only 50 independent samples. The standard errors of the scalar fields were used with the least-square fits to provide uncertainty of the lateral gradient quantities. We evaluated (2.17) at the interpolated point centered within the HRP survey grid. These central estimates are then used with the net diffusivities given by (2.14).

Estimates of the diapycnal advection are given in table 2.2 and plotted in figure 2.14. The epineutral contribution to $w_{*}\left(w_{*}^{(e p i)}\right.$, the term in (2.16) involving $\left.\kappa\right)$ turns out to be small, as is shown in figure 2.14a. The cabbeling term is nonzero in the few layers with nonzero $w_{*}^{(e p i)}$. The thermobaric term is generally very small. The diapycnal flux contribution to $w_{*}\left(w_{*}^{(d i a)}\right.$, the term in (2.16) involving the vertical diffusivities) was calculated using the net diffusivities given by table 2.1 and is shown in figure $2.14 \mathrm{~b}$. We do not report estimates for the upper and lower layers due to the use of a central difference in computing the divergence of the vertical fluxes. The uppermost layer with reported $w_{*}^{(d i a)}$ corresponds to $150 \mathrm{~m}<\bar{z}<250 \mathrm{~m}$. The diapycnal velocity in this layer is known very poorly, owing to the large uncertainty of the surface-layer fluxes. Figure $2.14 \mathrm{c}$ shows the sum of $w_{*}^{(e p i)}$ and $w_{*}^{(d i a)}$, giving the total diapycnal advection $w_{*}$. Two layers $\bar{z}=300 \mathrm{~m}$ and $\bar{z}=400 \mathrm{~m}$ are characterized by negative (downward) advection. In particular, the $\bar{z}=300 \mathrm{~m}$ surface shows downward advection of $w_{*}=-(1.7 \pm$ 1.2) $\mathrm{m} \mathrm{yr}^{-1}\left(w_{*} \approx-6 \times 10^{-8} \mathrm{~m} \mathrm{~s}^{-1}\right)$. A value for the diapycnal velocity in this layer was also reported by Ledwell et al. (1998), based on sinking of the tracer distribution relative to the release isopycnal. The Ledwell et al. (1998) estimate of $w_{*}=-(3 \pm 1) \mathrm{m} \mathrm{yr}^{-1}$ is shown in the figure and is within one standard error of our estimate.

We now contrast diapycnal advection estimates presented above with estimates based on an alternate approach. The Osborn (1980) model was used to calculate a net diffusivity $k_{0}$, based on the assumption that dissipation is solely due to turbulence. With no salt-finger enhancement of $k_{s}$, all scalars have the same vertical diffusivity, $k_{\theta}=k_{s}=k_{0}$, and the diapycnal-flux divergence components of the diapycnal advection can be expressed as

$$
\begin{aligned}
w_{*}^{(d i a)} \frac{g}{\bar{N}^{2}} & =\left(g^{-1} \sigma_{z} \frac{\partial}{\partial \sigma}\left(k_{0} \overline{N^{2}}\right)-k_{0} \frac{\partial \alpha}{\partial \theta}{\overline{\Theta_{z}}}^{2}\right) \\
& =\left(g^{-1} \Gamma^{(t)} \sigma_{z} \frac{\partial \epsilon}{\partial \sigma}-\frac{\partial \alpha}{\partial \theta} \frac{\alpha\rangle}{2}\right)
\end{aligned}
$$




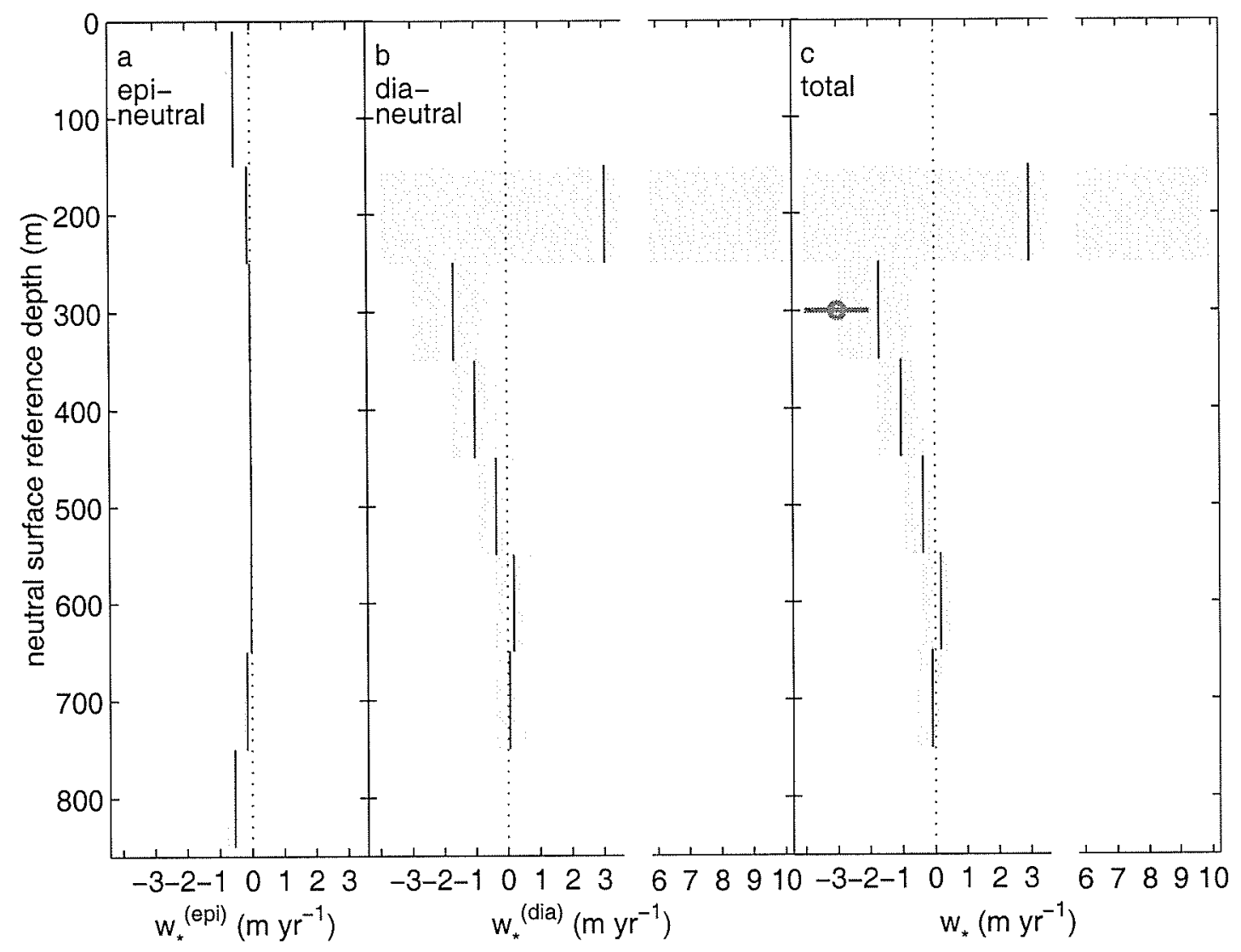

Figure 2.14: The diapycnal advection $w_{*}$ computed using the profiles of $k_{\theta}$ and $k_{s}$ shown in figure $2.13 \mathrm{~b}$. (a) The epineutral component of the diapycnal advection. This small contribution comes mainly from the cabbeling term. (b) The diapycnal-flux component of the diapycnal advection. Only layers at $\bar{z}=300 \mathrm{~m}$ and $\bar{z}=400 \mathrm{~m}$ have contributions distinguishable from zero. (c) The total diapycnal advection $w_{*}=w_{*}^{(e p i)}+w_{*}^{(d i a)}$. The downward advection at the $\bar{z}=300 \mathrm{~m}$ surface is consistent with the tracer-derived result (Ledwell et al. 1998) shown as the circle with error bar. 


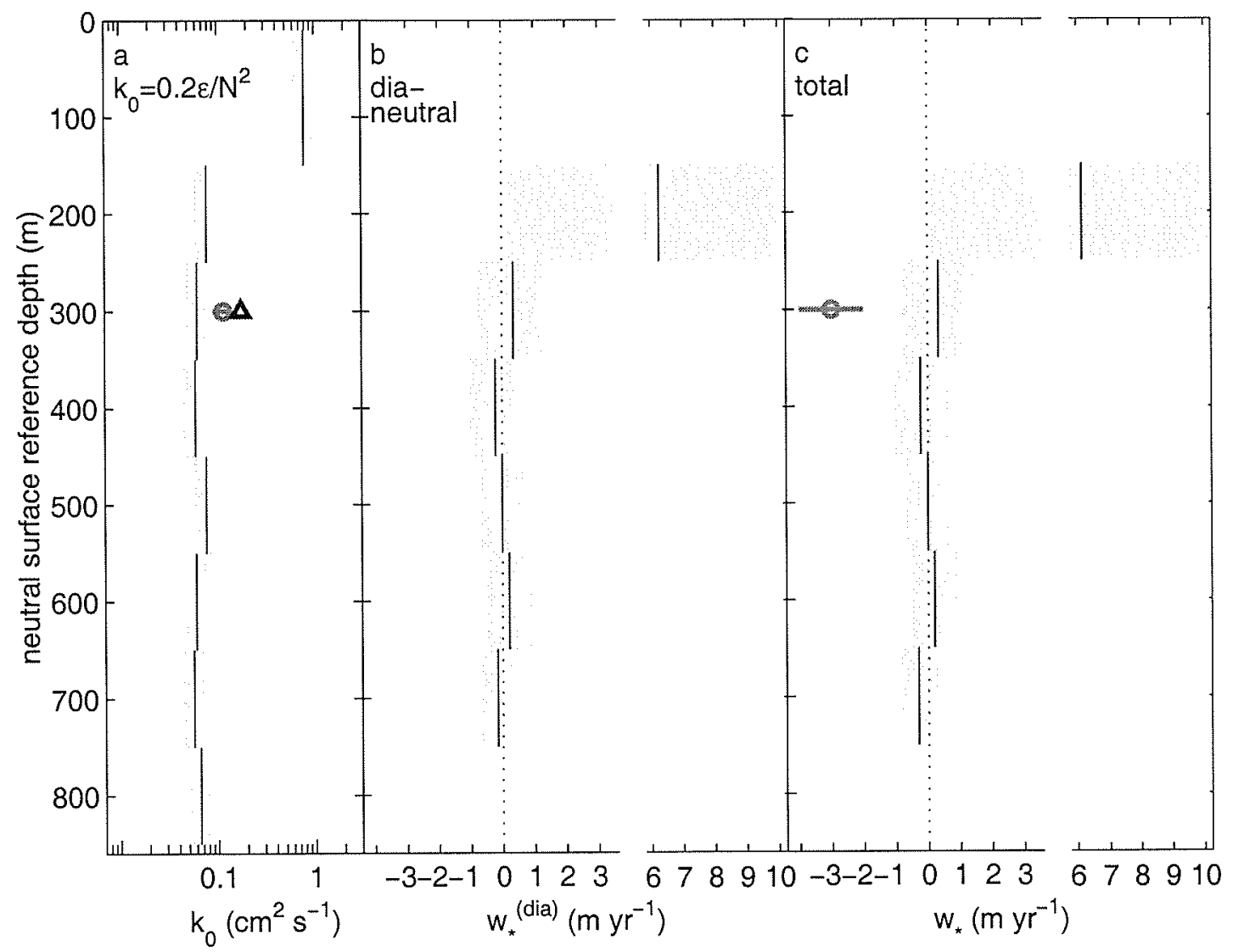

Figure 2.15: The diapycnal advection $w_{*}$ when no salt-finger enhancement of the haline diffusivity is used. (a) The scalar diffusivity of turbulence, $k_{0}=0.2\langle\epsilon\rangle$ $\left(\overline{N^{2}}\right)^{-1}$ (Osborn 1980). The tracer-derived diffusivities for May through November 1992 (circle) and November 1992 through November 1994 (triangle) are shown at the $\bar{z}=300 \mathrm{~m}$ surface. (b) The diapycnal contribution to the advection. (c) The total diapycnal advection (with the epineutral component from figure 2.14a). With no salt-finger enhancement of $k_{s}$, the advection at the tracer-release depth is no longer distinguishable from zero. 


\begin{tabular}{|c|c|c|c|c|c|}
\hline $\begin{array}{c}z \\
(\mathrm{~m})\end{array}$ & $\begin{array}{l}\frac{g \alpha \sigma_{z}}{\overline{N^{2}}} \frac{\partial k_{\theta}}{\partial \sigma} \overline{\Theta_{z}} \\
\left(\mathrm{~m} \mathrm{yr}^{-1}\right)\end{array}$ & $\begin{array}{l}\frac{g \alpha \sigma_{z}}{\overline{N^{2}}} k_{\theta} \frac{\partial \overline{\Theta_{z}}}{\partial \sigma} \\
\left(\mathrm{m} \mathrm{yr}^{-1}\right)\end{array}$ & $\begin{array}{r}=\frac{g \beta \sigma_{z}}{N^{2}} \frac{\partial k_{s}}{\partial \sigma} \overline{S_{z}} \\
\left(\mathrm{~m} \mathrm{yr}^{-1}\right)\end{array}$ & $\begin{array}{l}\frac{-g \beta \sigma_{z}}{\bar{N}} k_{s} \frac{\partial \overline{S_{z}}}{\partial \sigma} \\
\left(\mathrm{m} \mathrm{yr}^{-1}\right)\end{array}$ & $\begin{array}{c}w_{*} \\
\left(\mathrm{~m} \mathrm{yr}^{-1}\right)\end{array}$ \\
\hline 190 & 14.70 & -0.25 & -11.32 & -0.07 & 2.96 \\
\hline 300 & 2.39 & 1.62 & $-3 . .20$ & -2.49 & -1.71 \\
\hline 400 & 0.25 & 0.93 & -0.84 & -1.35 & -1.03 \\
\hline 500 & 0.28 & 1.22 & -0.50 & -1.36 & -0.36 \\
\hline 600 & 0.25 & 0.17 & 0.11 & -0.33 & 0.18 \\
\hline 700 & -0.20 & 0.04 & 0.35 & -0.13 & -0.09 \\
\hline
\end{tabular}

Table 2.3: Flux components of the dianeutral advection.

where the relations $k_{0} \overline{N^{2}}=\Gamma^{(t)}<\epsilon>$ and $k_{0}{\overline{\Theta_{z}}}^{2}=0.5<\chi>$ have been used. Figure 2.15 shows the alternate estimates of diapycnal advection. The net diffusivity estimated by $k_{0}=0.2<\epsilon>\left(\overline{N^{2}}\right)^{-1}$ is shown in figure 2.15a. This diffusivity is slightly less than the previously estimated $k_{\theta}$ and $k_{s}$. Figure $2.15 \mathrm{~b}$ shows the diapycnal component of the advection, given by (2.18). The diapycnal component of the advection was added to the previously estimated epineutral component and the total diapycnal velocity is shown in figure $2.15 \mathrm{c}$. This approach dictates that all layers beneath the influence of the mixed layer have diapycnal advection indistinguishable from zero. The best agreement between the microstructure- and tracer-derived mixing rates is achieved when the salt-finger enhancement of $k_{s}$ is accounted for.

\subsection{Discussion}

We have shown that elevated dissipation events are related to stability parameters characterizing the locale where the mixing is occurring. Elevated $\chi(\chi>1 \times$ $10^{-9} \mathrm{~K}^{2} \mathrm{~s}^{-1}$ ) can be associated with either turbulence or salt fingers based on the local values of the density ratio or Richardson number. Dissipation rates $\chi$ and $\epsilon$ were examined with respect to the two processes through the use of the nondimensional dissipation ratio $\Gamma$. Approximately $70 \%$ of the elevated $\chi$ data was consistent with the dissipation ratio of turbulence. These observations were generally associated with $R i<1$ (65\% of the elevated $\chi)$. Data with $\left(R_{\rho}>\right.$ $2, R i>1)$ were also characterized by $\Gamma$ consistent with the turbulence model. The remaining elevated $\chi$ occurred when $\left(R_{\rho}<2, R i>1\right)$. The dissipation ratio 
of these observations was inconsistent with the turbulence model. Instead, $\Gamma$ of the $\left(R_{\rho}<2, R i>1\right)$ data was found to be consistent with the model for fingers. Thus, when the Richardson number exceeds unity, there is a general transition at $R_{\rho} \sim 2$ between a turbulent-dominated and finger-dominated regime. Only $3 \%$ of elevated $\chi$ observations fall outside the generalizations referred to above. Observations binned in the parameter range $\left(1.1<R_{\rho}<1.3,0.3<R i<1\right)$ are consistent with salt fingers, while the $\left(2<R_{\rho}<4,10<R i<30\right)$ observations were inconsistent with either model.

The dissipation ratio analysis clearly rejects the notion that salt fingers are important throughout the general range of finger-favorable stratification $(1<$ $\left.R_{\rho}<100\right)$. On the contrary, most elevated $\chi$ data meet the dissipation ratio criterion for turbulence. However, evidence for fingers is strong when the stability parameters fall in the range $\left(1<R_{\rho}<2, R i>1\right)$. Our finding, that fingers are constrained to this narrow range of density ratio and Richardson number, supports conclusions of previous studies. Schmitt and Evans (1978) argued that growing modes of the finger instability overcome wave-strained gradients of $\Theta$ and $S$ only when $R_{\rho}<2$. Schmitt (1981) pointed out that the occurrence of thermohaline staircases is confined to $R_{\rho}<1.7$. Additionally, Linden (1974) used theory and experiment to show that weak shear permits fingers in the form of sheets aligned with the flow. Kunze (1994) has considered time-dependent shear and found that finger-sheets will be tilted as the shear vector turns with time. However, at $R_{\rho}<2$, vertical finger-fluxes remain strong.

The formal examination of the parameter space $\left(R_{\rho}, R i, \Gamma\right)$ presented here included only elevated dissipation rate observations of the form $\chi>1 \times 10^{-9} \mathrm{~K}^{-2} \mathrm{~s}^{-1}$. For elevated levels of $\chi$, there is a partition between finger and turbulence regimes. However, since the distributions of $\epsilon$ and $\chi$ are roughly lognormal, the exceptional events dominate the ensemble average. These ensemble averages dictate the magnitude of the vertical diffusivities. Thus, we expect the conclusions drawn about the association of salt fingers and turbulence with the stability parameters to apply not only to exceptional dissipation, but more generally to average dissipation.

We have implemented a method for estimating the diapycnal fluxes driven by both turbulent and salt-finger mixing events. The calculations involve partitioning observations of $\epsilon$ and $\chi$ into finger-favorable and turbulent conditions 
using the local values of $R_{\rho}$ and $R i$. For each class of dissipation, we calculate the thermal diffusivity using the production-dissipation balances (2.1) and (2.2). The haline diffusivity is set equal to $k_{\theta}$ for the ensemble of data not favorable to fingers. However, $k_{s}$ for the finger-favorable ensemble is calculated by enhancing the thermal diffusivity by the factor $R_{\rho} / r$, with $r=\left(\alpha k_{\theta} \overline{\Theta_{z}}\right)\left(\beta k_{s} \overline{S_{z}}\right)^{-1}$ known from laboratory measurements of salt fingers to be $r=0.6 \pm 0.04$ when $R_{\rho}<2$. A model for enhancing the haline flux in a region experiencing both turbulence and fingers was proposed by McDougall and Ruddick (1992, here after MR92). As we have done in (14), the MR92 model expresses vertical fluxes in terms of a sum between the turbulent and salt-finger derived dissipation. The MR92 model relies on algebraic relations between $\Gamma, \Gamma^{(t)}$, and $\Gamma^{(f)}$ to give the relative weights between the turbulent and finger-derived fluxes; we found this approach overly sensitive to uncertainty in the dissipation rate estimates. In contrast, in our approach the weighting factors $P^{(t)}$ and $P^{(f)}$ are determined directly from observations of $R_{\rho}$ and $R i$. Our analysis of the dissipation ratio was not critically dependent on the values for $\Gamma^{(t)}$ and $\Gamma^{(f)}$. Instead, our analysis served to show that a reasonable relationship exists between $R_{\rho}, R i$ and the dissipation ratio. We believe that our method for calculating diffusivities is the more relevant application of the information available in profiles of fine- and microstructure variables.

Our estimates of vertical diffusivities imply that salt fingers act to enhance $k_{s}$ over $k_{\theta}$ by about $60 \%$ at depths near the NATRE tracer-release surface. These diffusivities were used to calculate the diapycnal fluxes of heat and salt, and the divergences of these fluxes dictate the diapycnal advection. In both layers found to have downward advection $(\bar{z}=300 \mathrm{~m}$ and $\bar{z}=400 \mathrm{~m})$, the divergence of the haline-flux dominates over the thermal-flux divergence. To better understand the physical processes that determine $w_{*}$ we have dissected the diapycnal-flux divergence terms in the diapycnal advection equation into the form

$$
w_{*}^{(d i a)} \frac{g}{\sigma_{z} \overline{N^{2}}}=\alpha \frac{\partial k_{\theta}}{\partial \sigma} \overline{\Theta_{z}}+\alpha k_{\theta} \frac{\partial \overline{\Theta_{z}}}{\partial \sigma}-\beta \frac{\partial k_{s}}{\partial \sigma} \overline{S_{z}}-\beta k_{s} \frac{\partial \overline{S_{z}}}{\partial \sigma} .
$$

Thus, the divergence of the diapycnal fluxes are written as two terms: a term proportional to the derivative of the diffusivity and a term proportional to the derivative of the respective heat or salt gradient. The values of these terms are given in table 2.3. Advection through the $\bar{z}=300 \mathrm{~m}$ surface is most influenced 
by the vertical change of $k_{s}$, while the $\bar{z}=400 \mathrm{~m}$ surface is more determined by the vertical change of $\overline{S_{z}}$. In these layers, salt extracted from above increases the density of the resident parcels causing them to sink. This occurs despite the fact that resident parcels are also being heated. In this manner, salt-finger density fluxes act to oppose the traditional view of upwelling in the thermocline (Robinson and Stommel 1959).

Several models have been proposed that parameterize salt-finger fluxes in terms of the density ratio. The Schmitt (1981) model is ad hoc, with a power-law dependence that allows a considerable enhancement of $k_{s}$ at low density ratios. One aspect of the Schmitt (1981) model is the tendency for finger fluxes to maintain a tight $\Theta-S$ relation. Supporting evidence for this is found in the comparison of 5-m $R_{\rho}$ with the $\mathrm{O}(100 \mathrm{~m}) R_{\rho}$ in figure 2.13. These data suggest that the $\Theta-S$ relation is tightest in the depth range $200 \mathrm{~m}<\bar{z}<600 \mathrm{~m}$ where the fingers are most active. Zhang et al. (1998) have refined the Schmitt (1981) model such that the haline diffusivity is given by $k_{s}=\left(1 \times 10^{-4} \mathrm{~m}^{2} \mathrm{~s}^{-1}\right)\left(1+\left[R_{\rho} / 1.6\right]^{6}\right)^{-1}$. Another set of models which predict finger-fluxes subject to a dynamical constraint have been proposed by Kunze $(1987,1994)$. These models use a finger Richardson number constraint which is similar to the Stern (1969) Reynolds number constraint. The dissipation data from NATRE provides an opportunity to examine the applicability to these models to thermocline mixing. We have calculated finger-driven haline diffusivities using (2.12) and (2.13) with the 5 -m scale $\Theta-S$ gradients. These diffusivities are compared with diffusivities predicted by Zhang et al. (1998) and Kunze $(1987,1994)$ in figure 2.16 . The Kunze $(1987,1994)$ models predict an increase in diffusivity with $R_{\rho}$ not exhibited by the NATRE data. The relation used by Zhang et al. (1998) is a bit more diffusive than the data suggest, as may be appropriate for the larger-scale gradients used by the model, but has a density ratio dependence that is qualitatively correct.

In terms of the vorticity budget for the upper thermocline, the diapycnal advection we have documented would play a limited role. Specifically, "stretching" of fluid columns in the upper ocean given by $f \partial_{z} w_{(t o t a l)}$ would be dominated by the adiabatic component of vertical velocity, $\underline{u} \cdot \nabla z_{n}$, which is set by the Ekman pumping through the mixed layer. Joyce et al. (1998) estimate the Ekman downwelling applicable to the NATRE region to be $\sim 40 \mathrm{~m} \mathrm{yr}^{-1}$. Thus, the diabatic 


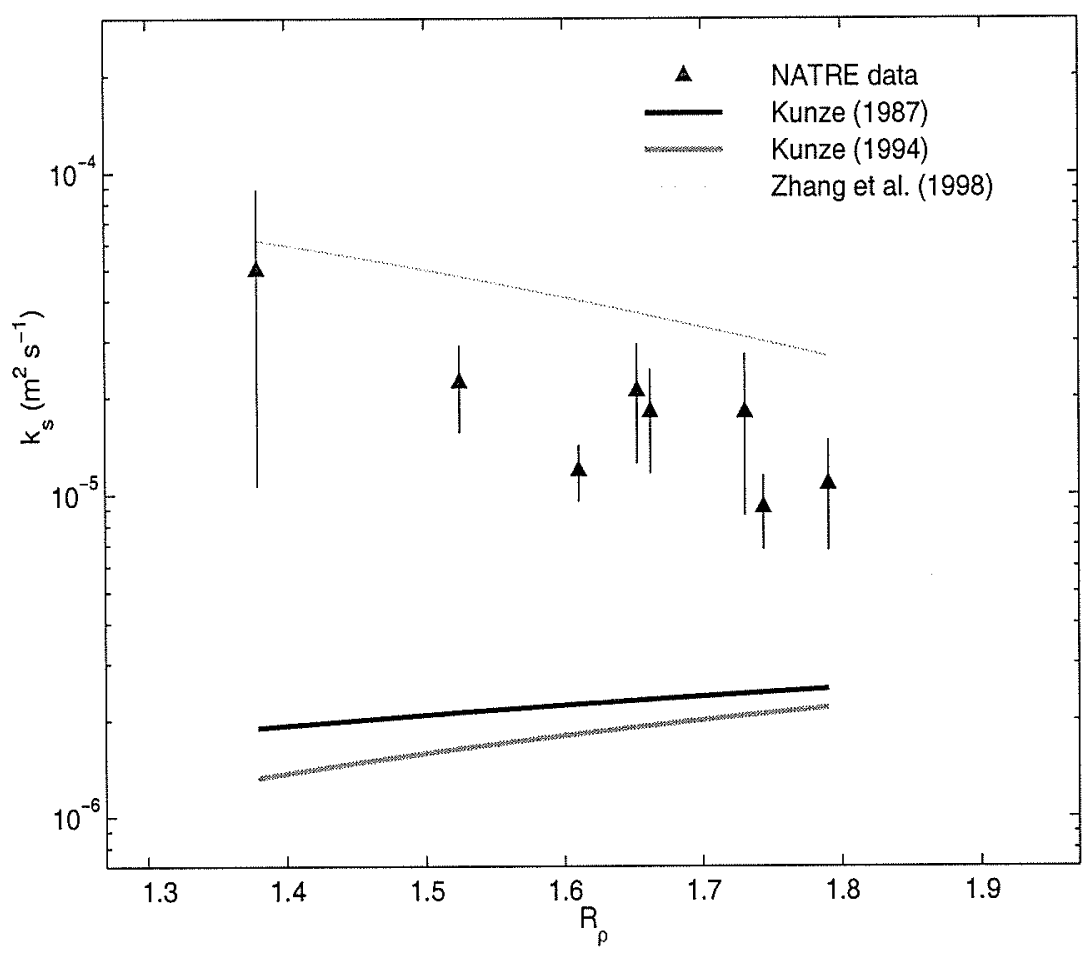

Figure 2.16: Comparison of the finger diffusivities for salt as estimated by the NATRE microstructure and salt-finger models. The Kunze $(1987,1994)$ models are based on stability considerations. The Zhang et al. (1998) model is based on the the Schmitt (1981) ad hoc model. 
forcing we have discussed comprises $\leq 10 \%$ of the total vertical velocity in the ventilated thermocline.

However, at depths not influenced by Ekman pumping, diabatic forcing will dominate the total vertical velocity and the vortex stretching. In particular, the underside of the Mediterranean property core is strongly favorable to salt fingers. Salt-finger fluxes in this region would drive downward advection beneath the core. Spall (1999) has examined the circulation in this region and found that vortex stretching by salt-finger driven advection would produce a circulation consistent with observations. Furthermore, the diapycnal fluxes we have documented must be of consequence to the long time-scale density budget. Models of the thermohaline circulation suggest that the efficiency of the oceanic heat transport is strongly influenced by differences between the vertical diffusivities $k_{\theta}$ and $k_{s}$ (Gargett and Ferron 1996; Zhang et al. 1998). In particular, these models find that salt fingering in the upper thermocline decreases the strength of the meridional overturning cell. This is because salt fingers drive an up-gradient density flux, and up-gradient density flux is detrimental to the vertical advective-diffusive balance that closes the thermohaline cell. As demonstrated by the NATRE data, the microstructure observations allow for estimation of the salt-finger component of the mixing. These salt-finger estimates are crucial in explaining the downward diapycnal-advection of tracer, as turbulent mixing alone can not account for an up-gradient density flux. Thus, we suggest that expanded use of the techniques developed here will be important in determining the influence of salt fingering on the general circulation. 


\section{Chapter 3}

\section{Buoyancy Forcing Above Rough Bathymetry in the Abyssal Brazil Basin}

\subsection{Introduction}

The densest waters of the ocean are formed at several high-latitude sites where winter temperature and salinity conditions favor static instability of the full water column. These waters spill into the abyss and are carried equatorward by Deep Western Boundary Currents (DWBCs). This view was proposed by Stommel (1957), and a model proposed by Stommel and Arons (1960) described a simple set of dynamics that links the DWBCs to a weak interior circulation. Being far removed from the wind driven Ekman layer, flow in the abyss is driven by buoyancy forcing. Lacking any information on the buoyancy forcing, Stommel and Arons calculated the circulation resulting from uniform upwelling. Under the uniform upwelling scenario, poleward flow dominates the interior.

The Stommel-Arons model is still central in our view of the abyssal circulation problem. The important roles of DWBCs and buoyancy forcing in the abyss are not questioned. However, mesoscale eddy phenomena have been known to significantly influence direct measures of deep flow since the float work of Swallow (1971). Furthermore, the nature of the interior flow is dependent on the specific distribution of deep sources and sinks of buoyancy. For this reason, estimates of abyssal 
mixing-rates have been the focus of numerous studies. In particular, hydrographic measurements can be used to formulate closed heat and mass budgets for deep semi-enclosed basins. Such heat-mass budgets have been the primary means of assessing abyssal mixing-rates, and vertical diffusivities of $\mathrm{O}\left(1-10 \mathrm{~cm}^{2} \mathrm{~s}^{-1}\right)$ have been reported in the North and South Atlantic (Saunders, 1987; Whitehead and Worthington, 1982), the South Pacific (Roemmich et al. 1996) and the Indian Ocean (Barton and Hill, 1989). These mixing rates are up to several orders of magnitude larger than typical thermocline values. In contrast to the mixing rates implied by budgets, direct observations of dissipation over abyssal plains suggest diffusivities there are comparable to thermocline levels (Gregg, 1977; Moum and Osborn, 1986; Toole et al., 1994; Kunze and Sanford, 1996). Instead, the larger mixing rates needed to reconcile the estimates of budgets seem to occur near rough bathymetry, as suggested by several studies (Toole et al., 1994, 1997a; Polzin et al., 1997).

Of specific interest to the present work is the South Atlantic, where the densest waters of the global ocean are formed in the Weddell Sea off Antarctica. These waters, so-called Antarctic Bottom Water (AABW), flow north along the western side of the South Atlantic reaching the Brazil Basin via the deep passages of the Vema and Hunter channels. The isotherm bounding the water colder than about $1^{\circ} \mathrm{C}$ intersects the sea floor within the basin. Thus, the coldest class of AABW does not exit the Brazil Basin adiabatically. The diffusion of heat associated with turbulent mixing provides the buoyancy needed to warm and upwell this water. The Brazil Basin budget of AABW was considered by Hogg et al. (1982), and revisited by Morris et al. (1997). These investigations indicate that a basinaverage vertical diffusivity of $2-4 \mathrm{~cm}^{2} \mathrm{~s}^{-1}$ is necessary to account for the basin's heat and mass budget.

To investigate the details of mixing in the abyssal Brazil Basin, a combined tracer and microstructure program was begun in 1996: The Brazil Basin Tracer Release Experiment (BBTRE). The microstructure data were collected during the tracer injection phase in January 1996, and again during the 14 month tracer inventory in April 1997. A free falling, autonomous instrument-platform called High Resolution Profiler was used (HRP; Schmitt et al., 1987). The collective microstructure observations spanned nearly $30^{\circ}$ longitude, covering the western 
basin and extending to the fracture zones of the Mid Atlantic Ridge (MAR). A preliminary presentation of microstructure-derived dissipation rates from the 1996 survey was given by Polzin et al. (1997), and data from the 1997 survey was discussed by Toole et al. (1997b). An additional description of the dissipation data, including a presentation of tracer results through the 1998 inventory, is given by Ledwell et al. (1999). While these previous reports have focused on the estimation of a turbulent diffusivities for the deep water of the Brazil Basin, the focus here will be to assimilate the dissipation data into an inverse model for the steady-state heat, mass and vorticity budgets.

The circulation problem will be posed in terms of isopycnal flow $(\underline{u})$ and diapycnal flow $\left(w_{*}\right)$. The budgets for heat and mass will be assessed within a series of potential-density layers. In much of the flow domain, geostrophic dynamics will be assumed and the lateral flow problem will be constrained through the use of the planetary vorticity equation

$$
\beta v=\frac{f}{h} \underline{u} \cdot \nabla h+\frac{f}{h}\left(w_{*}\left(z_{u}\right)-w_{*}\left(z_{l}\right)\right),
$$

where $h$ is the thickness of the density layer, and $\left(z_{u}, z_{l}\right)$ denote the upper and lower bounding isopycnal surfaces. The above expression is the density-layered version of the Stommel and Schott (1977) beta-spiral equation, and is discussed by McDougall (1988) and Hautala and Riser (1993). It permits solution of the "level of no-motion" problem by linear inversion when the geopotential-anomaly field has been measured. The diapycnal-advection term can modify the planetary vorticity by vortex stretching of fluid columns, and this diabatic term is related to the divergence of buoyancy flux $\left(J_{b}\right)$ and the buoyancy gradient $\left(N^{2}\right)$ by

$$
w_{*} N^{2}=-\frac{\partial J_{b}}{\partial z} .
$$

This expression for the diapycnal advection ignores processes related to the nonlinear thermodynamic-terms in the equation of state for seawater, described by McDougall (1984). Vertical exchanges of buoyancy accompany the dissipation of turbulent kinetic energy (TKE) during turbulent mixing events, and the energy budget for an ensemble of turbulent events is 


$$
\left(1-R_{f}\right) J_{b}+R_{f} \bar{\epsilon}=0,
$$

where $\epsilon$ is the dissipation rate, and $R_{f}$ is the efficiency of the mixing. The TKE budget expressed in (3.3) assumes steady-state and homogeneous statistics of the Reynolds stress tensor. This equation is discussed by Osborn (1980), and an expression for the eddy diffusivity is readily derived by substituting the Fickian diffusion term $J_{b}=-k_{\rho} N^{2}$ into (3.3) so that

$$
k_{\rho}=\left(\frac{R_{f}}{1-R_{f}}\right)\left(\frac{\bar{\epsilon}}{N^{2}}\right) .
$$

The fraction $R_{f}\left(1-R_{f}\right)^{-1}$ can be expressed as $-J_{b} / \bar{\epsilon}$, and the ratio of buoyancy flux to energy dissipated is typically taken to be $20 \%$ (Moum 1996). With use of (3.2) and (3.3), the diapycnal advection can be constrained by the dissipation data as

$$
w_{*} N^{2}=\left(\frac{R_{f}}{1-R_{f}}\right) \frac{\partial \bar{\epsilon}}{\partial z} .
$$

The value of this constraint is not limited to the geostrophic vorticity equation, but more generally to any advective budget. When considering flow near rough bathymetry, the geostrophic vorticity equation is of limited value, both due to the lack of a well measured geopotential-anomaly field, and also because the applicability of geostrophic balance is questionable in a topographic boundary-layer. However, the validity of (3.5) is independent of the geostrophic momentum balance, and (3.5) will serve as a primary hydrodynamic constraint when flow near abyssal topography is considered.

Previous attempts to examine mixing rates and diapycnal velocities in inverse models have been done for both box models of the advective budgets (e.g., Wijffels 1993; Tziperman and Hecht, 1988; Zhang and Hogg, 1992) and beta-spiral inversions (e.g., Olbers et al., 1985; Hautala and Riser, 1993; Wunsch 1994). These inversions all rely on either integrated property budgets (box inversions) or differential property budgets (beta-spiral inversions), and the mixing rates and diapycnal velocity estimates are determined through the divergences of lateral fluxes. This form of mixing-rate estimation is highly sensitive to small uncertainties in 
the advective terms, as is discussed by Wunsch (1996). The inverse model used in this study resembles the model of Fukumori (1991) in that both integrated and differential budgets are used. In this manner, mass conservation and the thermal advection are closed in volume-integrated cells, while the advection of planetary vorticity is examined in the differential form of the beta-spiral equation. The diffusivity and diapycnal advection are constrained in these budgets by application of (3.4) and (3.5).

The description of the turbulent dissipation observed during BBTRE will begin in section 3.2. There, we will discuss spatial and temporal features of mixing (section 3.2.1), a physical mechanism that accounts for the observations will be proposed (section 3.2.2) and a model relating $\epsilon$ to height above bottom (section 3.2.3). In section 3.3, the inverse model for examining steady circulation will be presented. There we will describe the governing equations for the model (section 3.3.1), the treatment of data (section 3.3.2) the details of the linear inverse (section 3.3.3), and the results of the calculation (section 3.3.4). In section 3.4, the influence of mixing on flow in an abyssal canyon will be considered. A discussion of the estimated circulation will be given in section 3.5 .

\subsection{Dissipation}

\subsubsection{Description of Dissipation Data}

In the period between January 1996 and April 1997, two microstructure surveys were made in the Brazil Basin. During the first visit in January through February 1996, a 75 station HRP survey with two section legs covered a region extending from the continental slope regions off Rio de Janeiro $\left(24^{\circ} \mathrm{S}\right)$ and Recife $\left(11^{\circ} \mathrm{S}\right)$ to the western edge of the MAR $\left(16^{\circ} \mathrm{W}\right.$ at $\left.21^{\circ} \mathrm{S}\right)$ where the tracer was injected. The second survey was carried out in March and April of 1997. As part of the effort to inventory the tracer patch, an irregular grid of stations were occupied over the domain $\left(12^{\circ} \mathrm{W}-23^{\circ} \mathrm{W}, 20^{\circ} \mathrm{W}-25^{\circ} \mathrm{S}\right)$. A primary focus of the 1996 survey was to establish the spatial variability of mixing levels across the basin's continental rise and fracture zone system. A report on the observed spatial variability is given by Polzin et al. (1997), and will generally not be discussed further here. 
Instead, the present work will focus on dissipation data collected over the fracture zones east of $25^{\circ} \mathrm{W}$. This data set consists of 87 stations from the 1997 survey and an additional 42 stations from the 1996 survey (figure 3.1). All profiles consist of conventional hydrographic variables (e.g., $\Theta, S$ ) in addition to microstructure, and generally extend from the surface mixed-layer to within $20 \mathrm{~m}$ of the bottom. The bathymetric data shown in figure 3.1 were derived from satellite measurements of the marine gravity-field (Smith and Sandwell, 1997). The map clearly shows the network of fracture zones (FZs) leading east to the MAR. This system of FZs is characterized by a series of canyons bounded latitudinally by ridges whose crests rise up to $1 \mathrm{~km}$ above the canyon floors. The canyon of an unnamed FZ between $21^{\circ} \mathrm{S}$ and $22^{\circ} \mathrm{S}$ was heavily sampled during the 1997 survey, and observations from this canyon will receive careful attention in section 3.4 .

The dissipation data were derived from observations of velocity microstructure. Only two components of the strain tensor are measured and isotropy is assumed to express the dissipation rate as $\epsilon=(15 / 4)\left(\left\langle u_{z}^{2}\right\rangle+\left\langle v_{z}^{2}\right\rangle\right)$. The use of the isotropy assumption can not be justified through inspection of the available field data, but studies have shown that the isotropy relation provides a good estimate of dissipation (Yamazaki and Osborn, 1990; Itsweire et al., 1993). The precise details of the dissipation calculation are discussed by Polzin and Montgomery (1996). In the presentation of data that follows, velocity-derived dissipation rates, versus those derived from temperature microstructure, are discussed exclusively. While high levels of shear microstructure characterize the bottom most $100 \mathrm{~m}$ in the canyons of the FZs, thermal microstructure levels there were very low and this precluded meaningful estimation of thermal dissipation $(\chi)$. However, the stratification was generally greater above the crests of the FZs where measurable levels of thermal dissipation were found, and in regions where the stratification was sufficient to resolve $\chi$, comparisons of the two dissipation rates were made. These comparisons indicated that the dissipation ratio, $\Gamma=\bar{\chi} N^{2}\left(2 \bar{\epsilon} \Theta_{z}^{2}\right)^{-1}$, was in the range expected for shear-produced turbulence, $0.05<\Gamma<0.3$. For this reason, we did not pursue evidence for double-diffusive processes. Furthermore, we have assumed that the dissipation ratio is related to the mixing efficiency by $\Gamma=R_{f}\left(1-R_{f}\right)^{-1}$, and that a meaningful estimate for the vertical diffusivities of heat, salt and buoyancy is given by $k=\left(\Gamma / N^{2}\right) \bar{\epsilon}$. 


\begin{tabular}{|cccccccccccc}
\hline 2000 & 2500 & 3000 & 3500 & $\begin{array}{c}4000 \\
\text { depth }(\mathrm{m})\end{array}$ & 4500 & 5000 & 5500 & 6000 & 6500
\end{tabular}

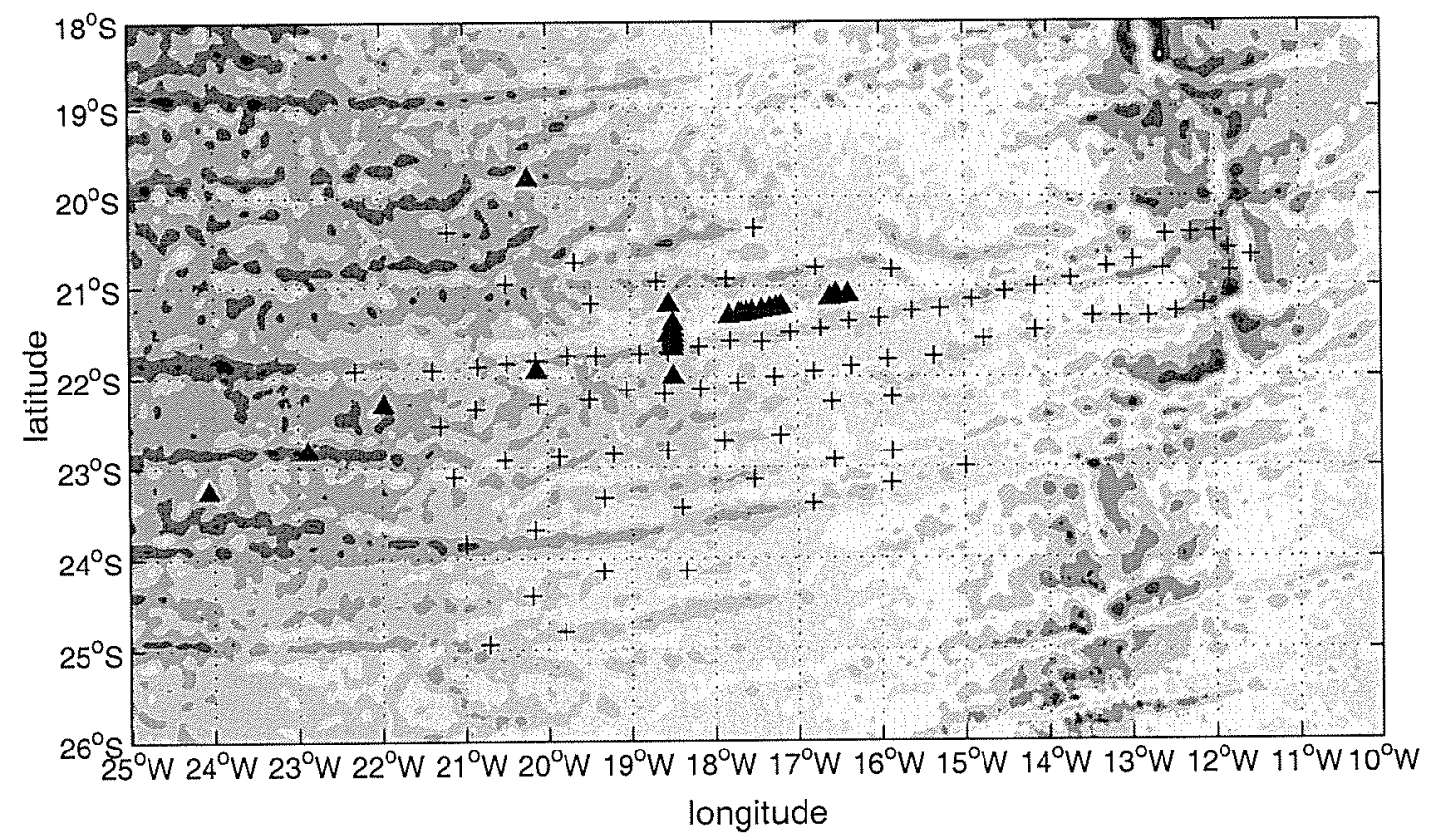

Figure 3.1: Map of the BBTRE site with the 2 arc-minute resolution bathymetric estimates of Smith and Sandwell (1997). A total of 129 profiles of dissipation rate observations were used from HRP profiles made during 1996 (triangles) and 1997 (crosses). 
As a means of presenting a summary of the observed deep-dissipation, a longitudinal section of $\epsilon$ was made by ignoring the latitudinal spread of the stations. This summary is presented in figure (3.2). To simplify the presentation, only the depth range deeper than $2000 \mathrm{~m}$ is shown. The dissipation data were depth averaged into $100 \mathrm{~m}$ intervals. Individual profiles are shown as columns of binned dissipation, with longitudinal widths that are inversely proportional to the density of station data. Since the latitudinal extent of the survey has been collapsed onto a single plane of longitude, no particular section of bathymetry is entirely appropriate for comparison. Instead, three traces of bathymetry are shown: the deepest, the shallowest, and the median values of the bottom depth for the latitude range $21^{\circ} \mathrm{S}$ to $23^{\circ} \mathrm{S}$.

Within about $500 \mathrm{~m}$ of the bottom, there is an enhancement of dissipation by up to several orders of magnitude over values observed at mid-depth. For each of the bathymetric traces shown in figure (3.2) there is an associated boundary layer of locally enhanced $\epsilon$. The signature of enhanced dissipation that tracks the trace of the shallowest bathymetry is associated with ridge crests. A deeper layer of enhanced $\epsilon$ follows the trace of the median topography and is associated with the sloping regions between canyons and crests, it is in this layer that the largest dissipation is observed.

There are multiple layers of enhanced dissipation, each associated with the different sloping regions that characterize the crest-canyon system of the FZs. This suggests that height above bottom $\left(h_{a b}\right)$ may serve as a better coordinate than depth for modeling the spatial distribution of $\epsilon$. This approach was adopted, and the available profiles of $\epsilon$ were classified according to the slope of the nearby bathymetry. Given the gridded estimates of bottom depth $Z_{b}(x, y)$, topographic slopes were categorized as either ridge crests $\left(\partial_{y} Z_{b} \sim 0, \partial_{y y} Z_{b}<0\right)$, canyon floors $\left(\partial_{y} Z_{b} \sim 0, \partial_{y y} Z_{b}>0\right)$, or sloping regions $\left(\partial_{y} Z_{b} \neq 0\right)$. The ensembled profile data, classified in this manner, are shown as functions of $h_{a b}$ in figure (3.3). Each ensemble profile results from data that has been vertically averaged into $100-\mathrm{m}$ bins. The $95 \%$ confidence interval of each bin was calculated using the "boot strap" Monte Carlo method (Efron and Gong, 1983). Dissipation decreases with height in each of the 3 ensembles, and all profiles reach "background" levels of dissipation, $\epsilon \sim 3 \times 10^{-10} \mathrm{~W} \mathrm{~kg}^{-1}$, at heights greater than $1000 \mathrm{~m}$. However, 


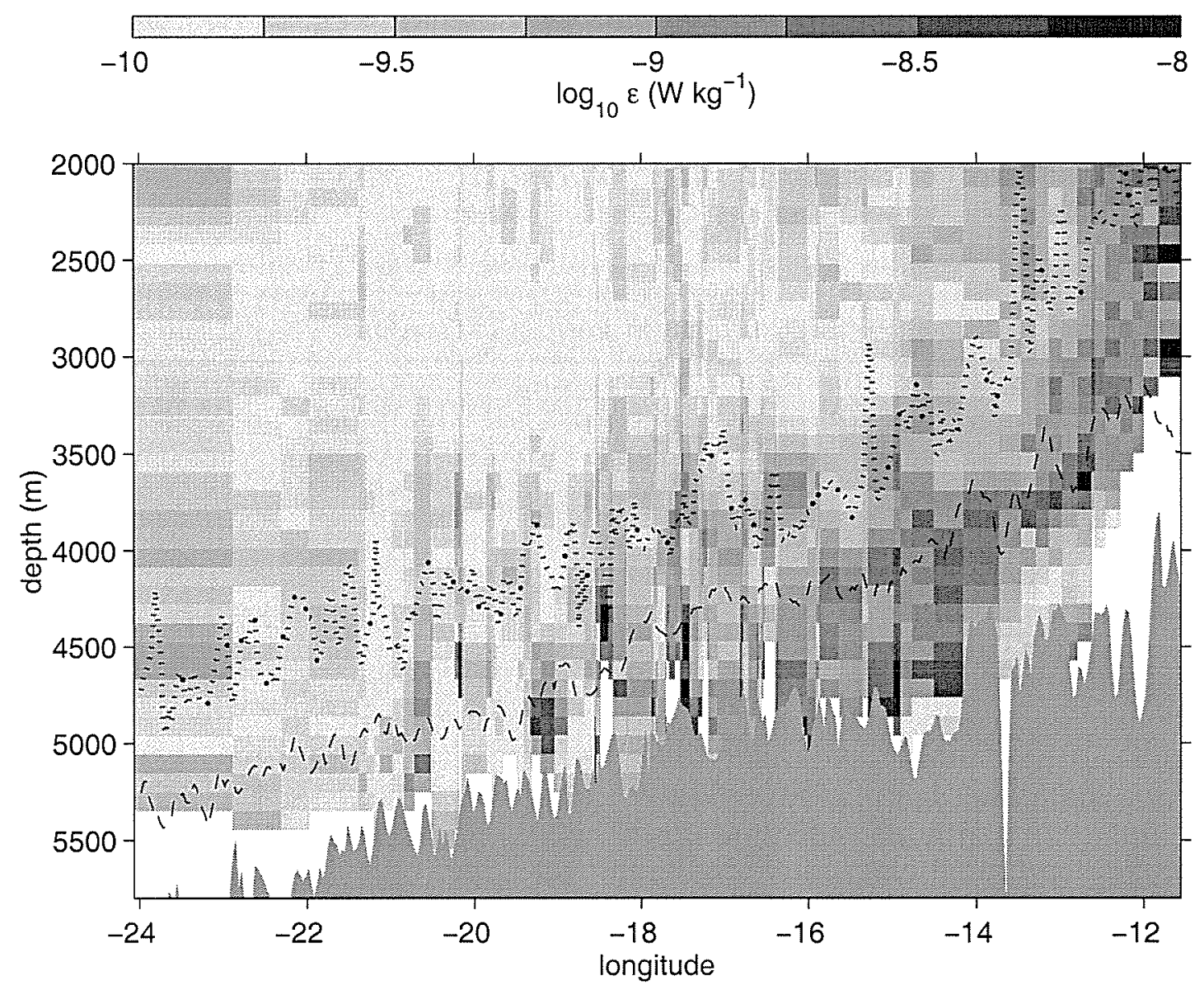

Figure 3.2: A zonal section of TKE dissipation $\epsilon$. The individual profiles are shown as columns, with each column's width being inversely proportional to zonal density of stations. Dissipation rate observations were vertically averaged into $100 \mathrm{~m}$ bins. The meridional distribution of stations was ignored to make this section. The shaded bathymetry is composed of the deepest estimated bottom depths between $\left(21^{\circ}-23^{\circ}\right) \mathrm{S}$, and is representative of canyon floors. The shallowest trace of bathymetry (dotted line) are the crests of ridges, as identified by the set of shallowest bottom depths, while the middle trace depicts the median level of sloping bathymetry along FZ walls. 


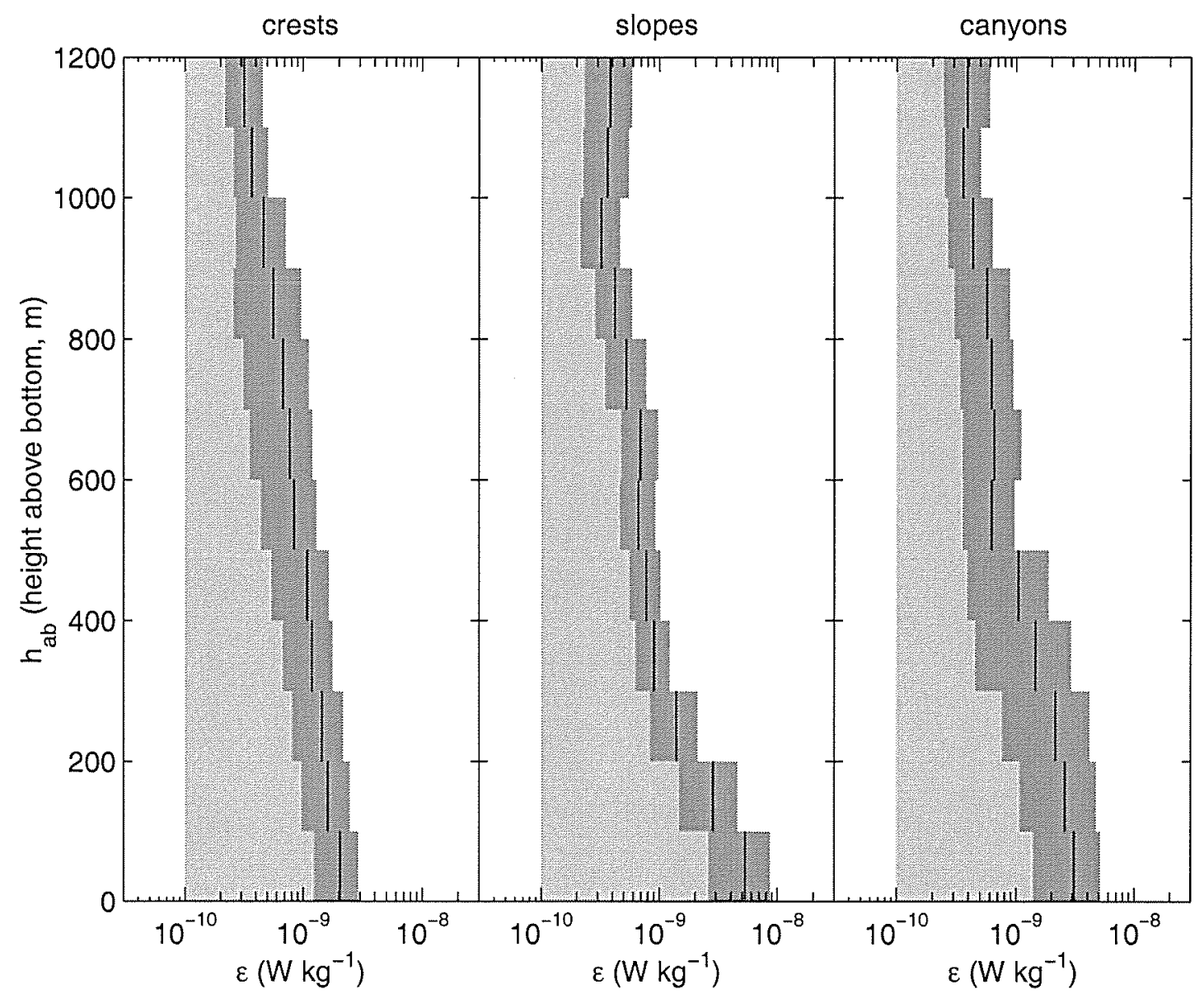

Figure 3.3: Dissipation profiles averaged according to the classification of nearby bathymetry $Z_{b}(x, y)$. Bathymetric classes were assigned as crests $\left(\partial_{y} Z_{b} \sim\right.$ $\left.0, \partial_{y y} Z_{b}<0\right)$, canyons $\left(\partial_{y} Z_{b} \sim 0, \partial_{y y} Z_{b}>0\right)$, or slopes $\left(\partial_{y} Z_{b} \neq 0\right)$. All profiles are shown relative to a reference dissipation of $\epsilon=1 \times 10^{-10} \mathrm{~W} \mathrm{~kg}^{-1}$ The $95 \%$ confidence interval is shown for each $100 \mathrm{~m}$ average. 
the profile for slopes is notably different in both the magnitude of the maximum dissipation and the scale of decay. The slope profile has a bottom value of $\epsilon \sim$ $(3-9) \times 10^{-9} \mathrm{~W} \mathrm{~kg}^{-1}$ and decays with an e-fold scale of $(150 \pm 50) \mathrm{m}$. The crest and canyon profiles peak at $\epsilon \sim(2-5) \times 10^{-9} \mathrm{~W} \mathrm{~kg}^{-1}$ and decay over a larger scale, $(500 \pm 100) \mathrm{m}$.

\section{Spatial Bias}

The factor of 2 to 4 difference in peak dissipation values, as well as the different decay scales, suggest that an average mixing-rate for a given level of depth will be dependent on the proximity of ridges, slopes and canyons. For example, a density surface that intersects sloping bathymetry will locally experience enhanced mixing, while a nearby point above the floor of a canyon, experiences background levels of dissipation. Thus, for a given depth, the appropriate average value of $\epsilon$ is weighted by the fraction of total area in contact with each class of bathymetry. A demonstration of this issue is presented in figure 3.4. The diagram shows a section of altimetry-derived bathymetry along the longitude of the tracer injection, $18.5^{\circ} \mathrm{W}$. The level of the tracer injection, with a target density of $\sigma_{4}=45.94 \mathrm{~kg} \mathrm{~m}^{-3}$, is also shown. At the latitude of the tracer injection $\left(21^{\circ} \mathrm{S}\right)$, the target surface is $600 \mathrm{~m}-1000 \mathrm{~m}$ above the floors of canyons. As is apparent in the figure, these heights above bottom correspond to dissipation values of $\epsilon=(1-10) \times 10^{-10} \mathrm{~W} \mathrm{~kg}^{-1}$. At other locations; the tracer surface is much closer to the bottom. In these places, the tracer surface experiences much larger values of dissipation.

Much of the data from the 1997 survey was collected above abyssal canyons. For density surfaces that reside above the crests of ridges, this implies that a simple average of $\epsilon$, made by accumulating data from the intersections of profiles with the density surface, will be biased low. To recover an unbiased estimate, the spatial distribution of crests, slopes and valleys must be used with the available dissipation data.

\section{Temporal Bias}

In addition to the spatial trend associated with height above bottom, a temporal modulation exists in the dissipation data. Polzin (1999) has proposed a model 


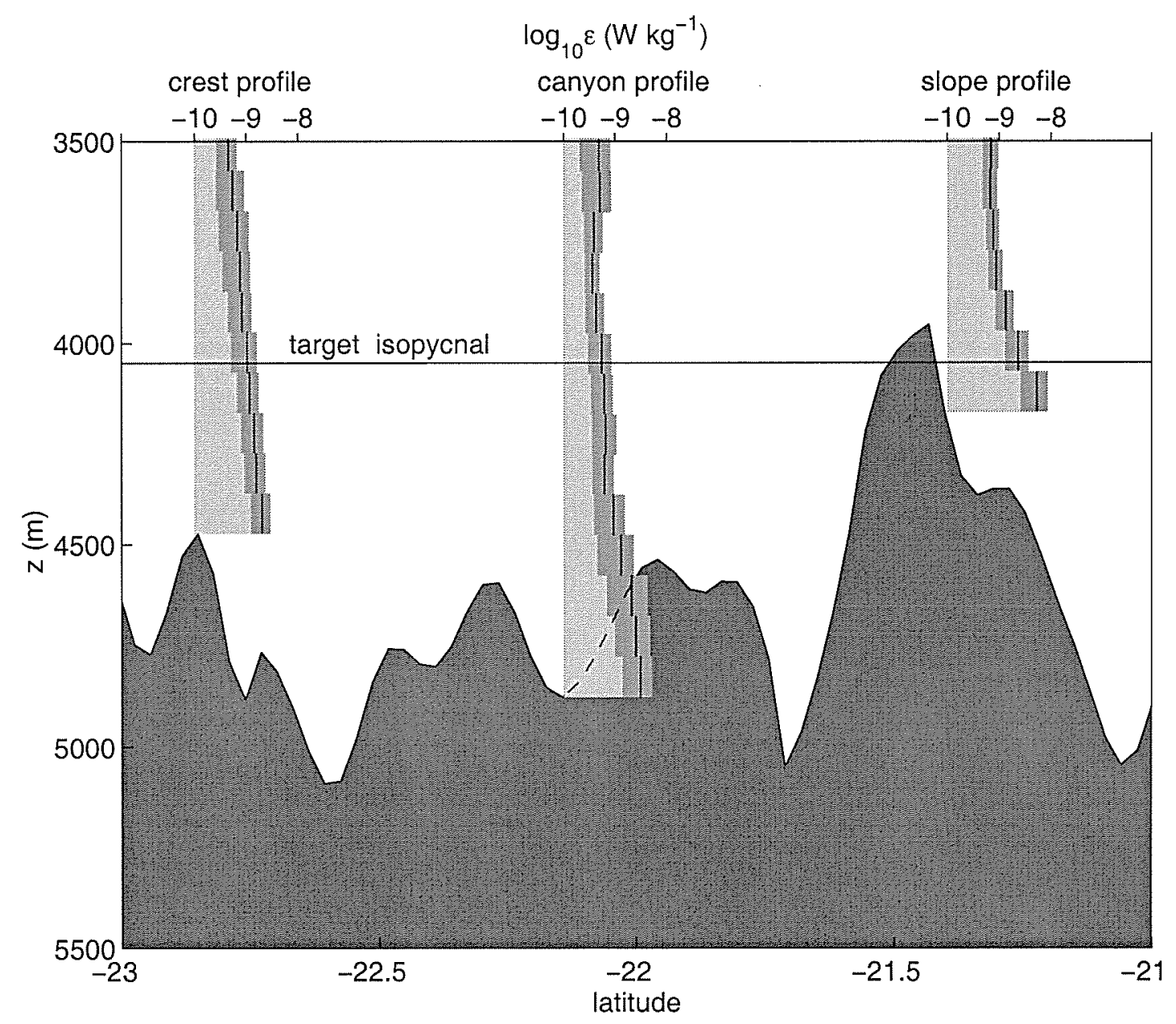

Figure 3.4: A section of meridional bathymetry at the longitude of the tracer injection $\left(18.5^{\circ} \mathrm{W}\right)$. The target isopycnal $\left(\sigma_{4}=45.94 \mathrm{~kg} \mathrm{~m}^{-3}\right)$ of the tracer is also shown, as are the ensemble-averaged dissipation profiles for the 3 classes of bottom bathymetry. The target isopycnal experiences enhanced mixing rates along the slopes and peaks of $F Z$ ridges. An estimate of mean dissipation for the target surface based on samples made above FZ canyons would be biased low. 
that suggests dissipation profiles like those shown in figure (3.4) are efficiently maintained by tidal forcing. This model motivated an examination of the temporal dependence of the data and a correlation between dissipation and the spring-neap cycle of tides was found. This correlation is most evident when the dissipation is vertically integrated. A comparison between integrated $\epsilon$ and tidal forcing was made by using estimates of barotropic tidal-velocity produced by the TPXO model of Egbert et al. (1994), and this is shown in figure 3.5. The tidal velocity data are expressed as a root-mean-square (rms) magnitude, and three powers of the tidal speed are shown. For the time intervals where dissipation data were available, the TPXO model was used to produce velocity estimates at the latitude and longitude appropriate for each HRP station. For the tidal signal during the intervals preceding and following the survey, the tidal current estimates at the tracer release site $\left(18.5^{\circ} \mathrm{W}, 21^{\circ} \mathrm{S}\right)$ ' are shown.

In both 1996 and 1997 comparisons, profiles of $\rho \epsilon$ were integrated to $h_{a b}=$ $2000 \mathrm{~m}$, and all profiles taken within 24 hours were averaged. The modulation of integrated dissipation follows the phase of the spring-neap cycle, with the possibility of the tidal forcing leading by about a day. The comparison of integrated dissipation with $\operatorname{rms}(u)^{n}$ is not the result of a fit. The dependent axes of the graphs have simply been arranged to situate the dissipation data in the middle of the three tidal curves. The exponents $n=2$ and $n=3$, were chosen based on simple ideas about mechanisms for the generation of bottom intensified turbulence, to be discussed in the section that follows. Correlation coefficients between the 1997 dissipation data and the three tidal curves are all near 0.7, although the correlations are much lower for the 1996 data. Furthermore, no specific power law dependence is strongly favored by a statistical analysis of this limited data: a linear fit of $\log \left(\int \rho \epsilon d h\right)$ to $\log (u)$ dictates powers between 1 and 3 are consistent with the data. Thus, a decision about a likely generation mechanism, and hence a likely power dependence on $\mathrm{rms}(u)$, must rely on physical considerations.

During the 1997 survey, nearly all of the sampling done west of $18^{\circ} \mathrm{W}$ occurred at neap tides. The sampling over the crests of the MAR occurred during spring tide. Furthermore, the collective average of our dissipation data favors neap tides, as this was the tidal phase for 3 of the 5 weeks over which the survey was done. Thus, the tidal correlation implied in figure (3.5) dictates that a simple average 
of observed $\epsilon$ will again be biased low.

\subsubsection{Mechanisms of Bottom Generation}

Having established a correlation between tidal forcing and turbulent dissipation, a physical mechanism that explains the dependence of $\epsilon$ on $\operatorname{rms}(u)$ is sought. Identification of a specific generation mechanism will aid in dealiasing the fortnightly variability in the data. Two mechanisms are considered, these being frictional boundary-layers and internal waves.

The kinematics of frictional boundary-layers are commonly used to model the turbulence that occurs when flow exerts stress on the seafloor. The work done by the bottom stress is assumed to balance the energy dissipation occurring in the boundary layer, and the expression for the work done by bottom drag is proportional to cubed velocity:

$$
W=\rho_{0} c_{d}\left|u_{\infty}\right| u_{\infty}^{2}
$$

where $c_{d} \sim 0.0025$ is the coefficient of drag, and $u_{\infty}$ is the uniform velocity of flow above the boundary layer (Trowbridge and Madsen, 1984). Taking $u_{\infty}=\operatorname{rms}(u)=$ $2 \mathrm{~cm} \mathrm{~s}^{-1}$, the work done by tides running over a frictional boundary layer is $W \sim 0.02 \mathrm{~mW} \mathrm{~m}^{-2}$. This number is two orders of magnitude smaller than the integrated dissipation occurring between the bottom and a height of $2000 \mathrm{~m}$. It is further noted that HRP profiles seldom extend close enough to the bottom to sample the frictional boundary layer, which is typically $\mathrm{O}(1 \mathrm{~m})$ thick. This work calculation demonstrates the insignificance of tidal driven bottom-stress.

Internal waves provide a more plausible mechanism for generating bottom intensified turbulence of the magnitudes observed. The problem associated with the production of internal waves when flow encounters bottom bathymetry is well known, and a general discussion is given by Gill (1980). Internal waves produced by steady flow over topography are termed "lee waves", although this term is used by Bell (1975a,1975b) for the problem of time dependent tidal flow. An expression for the energy input into the internal-wave field by flow over bathymetry can be derived from the linear wave equations. The rate at which energy is communicated to a point above the bottom is given by the vertical component of the wave energy- 



Figure 3.5: Comparison of column-integrated dissipation with power relations for the barotropic tidal speed. The rms tidal speed (solid line) is shown for general comparison purposes, but dynamical scalings suggest $\epsilon \sim u^{2}$ (dashed line) or $\epsilon \sim u^{3}$ (dotted line). The dissipation estimates $\rho \epsilon$ were integrated to $h_{a b}=2000 \mathrm{~m}$, and daily averages with standard errors are shown. The tidal velocity record was produced by the TPXO model of Egbert et al. (1994), and estimates were made along the survey tracks. Tidal estimates at a central location $\left(18.5^{\circ} \mathrm{W}, 21^{\circ} \mathrm{S}\right)$ were used for time intervals preceding and following survey periods. 
flux $\left(c_{g} E\right)$, and for quasisteady flow over cosine bumps this is

$$
\hat{z} \cdot c_{g} E=0.5 \rho_{0} \kappa u^{2} N h^{2}
$$

where $N$ is the buoyancy frequency and $(k, h)$ are the wavenumber and amplitude that characterize the bathymetry. The quasisteady limit is justified when the buoyancy response is faster than the time scale of the forcing, i.e. $N \gg \omega$. Bell (1975b) has examined the more general problem of tidal flow over bathymetry with spectral wavenumber dependence $\kappa^{-5 / 2}$, typical of some mid ocean ridges, and finds the vertical energy flux is proportional to $u^{5 / 2}$.

The connection between bottom generation of internal waves and dissipation relies on assumptions about the nonlinear interactions of internal waves of different scales. Polzin (1999) considers a model for the generation to dissipation process, and this model predicts dissipation profiles with character similar to the observations. The details of such a model need not be discussed here. Instead we assume that all energy fluxed away from the bottom is dissipated within some height $\delta$ of the bottom. This implies the energy balance

$$
\int_{0}^{\delta} d h \rho \epsilon=\hat{z} \cdot c_{g} E
$$

An estimate of the energy flux (3.6) was made for various assumptions about the bottom bathymetry. The frequency bandwidth of the generated internal waves is subject to $f^{2}<(\kappa u)^{2}<N^{2}$, and for values appropriate to the data $(f=$ $-5.4 \times 10^{-5} \mathrm{~s}^{-1}, N=9 \times 10^{-4} \mathrm{~s}^{-1}$, and $u=2 \mathrm{~cm} \mathrm{~s}^{-1}$ ), horizontal wavelengths between $100 \mathrm{~m}$ and $2.5 \mathrm{~km}$ are implicated. Taking a bathymetric amplitude of $50 \mathrm{~m}$ as typical of $1 \mathrm{~km}$ horizontal scales, the vertical energy-flux component carries $\mathrm{O}\left(1 \mathrm{~mW} \mathrm{~m}^{-2}\right)$. Thus, unlike the case of bottom friction, internal-wave radiation carries enough energy to feed the dissipation occurring within $2000 \mathrm{~m}$ of the bottom.

For completeness, the buoyancy work done by geothermal heating is also considered. Values of geothermal heat-flux $\left(F_{b o t}\right)$ vary from $50 \mathrm{~mW} \mathrm{~m}{ }^{2}$ across abyssal plains, to values of $150 \mathrm{~mW} \mathrm{~m}^{-2}$ near the axis of the MAR. These numbers seem very large when compared to the $1 \mathrm{~mW} \mathrm{~m}^{-2}$ values of integrated dissipation discussed above. However, this discrepancy is deceiving, as these large $F_{\text {bot }}$ values are 
not representative of their associated terms in the momentum equations. Consider the buoyancy flux $J_{b o t}$ produced by geothermal heating at the bottom boundary. The proper expression is derived by Joyce et al. (1986),

$$
J_{b o t}=\frac{g \alpha F_{b o t}}{\rho c_{p}}
$$

where $c_{p} \sim 4 \times 10^{3} \mathrm{~J}\left(\mathrm{~kg}^{\circ} \mathrm{C}\right)^{-1}$ is the specific heat of seawater. For a value of $F_{b o t}=$ $100 \mathrm{~mW} \mathrm{~m}{ }^{-2}$, the buoyancy flux by geothermal heating is $J_{b o t}=5 \times 10^{-11} \mathrm{~W} \mathrm{~kg}^{-1}$. This quantity competes in the TKE equation with the buoyancy production of turbulence, $J_{b}=0.2 \bar{\epsilon}$. Noting that observed dissipation rates along the bottom are $\bar{\epsilon} \sim 5 \times 10^{-9} \mathrm{~W} \mathrm{~kg} \mathrm{~kg}^{-1}$, buoyancy fluxes by mixing would exceed those by geothermal fluxes by at least an order of magnitude. The mechanical work done by the geothermal heating can be calculated as $W_{b o t}=\rho J_{b o t} \delta$, where $\delta$ is a boundary layer thickness. Taking $\delta$ as $2000 \mathrm{~m}, W_{b o t}=0.1 \mathrm{~mW} \mathrm{~m}^{-2}$ is to be compared to the $(1-3) \mathrm{mW} \mathrm{m}^{-2}$ of integrated dissipation observed. Clearly, liberal estimates for the role of geothermal heating dictate no more than a $10 \%$ influence.

\subsubsection{A Model for the Dissipation}

A model for the dissipation is needed to provide a constraint for diapycnal advection which will be used in the inverse calculations. The model is necessary in part because simple averages of the observed dissipation are biased low due to spatial and temporal dependencies. The spatial dependence is attributed to the enhancement of mixing that occurs along the slopes of ridge-canyon bathymetry, while the temporal dependence is related to a fortnightly modulation of vertically radiated internal-wave energy. By modeling the dissipation data, we aim to restore an unbiased average of the turbulent mixing rates.

A model for the dissipation was developed in several steps. First, the data were temporally dealiased assuming that $\epsilon \sim \alpha u(t)^{2}$, where a requirement is that $\alpha$ not be a function of time. A temporal weighting function can be defined that restores the time mean values of dissipation $(\hat{\epsilon})$ from the time dependent observations,

$$
\hat{\epsilon}\left(\underline{x}, h_{a b}(\underline{x})\right)=\left(\overline{u^{2}} / u(t)^{2}\right) \epsilon\left(\underline{x}, h_{a b}(\underline{x}), t\right),
$$


where $u(t)^{2}$ is barotropic tidal speed that has been smoothed with a one day running average. The long term average for $\overline{u^{2}}$ was computed over the 14 month period between the 1996 and 1997 surveys using tidal velocity estimates at the site of tracer injection, $\left(18.5^{\circ} \mathrm{W}, 21^{\circ} \mathrm{S}\right)$. The resulting temporally dealiased model of the dissipation is a function of spatial variables only. The second procedure in the modeling was to simplify the spatial dependence. This was done by categorizing the data into the three classes associated with bathymetry: ridge crests, canyons, and the slopes between them. The resulting model for dissipation is dependent only on $h_{a b}(\underline{x})$,

$$
\bar{\epsilon}\left(h_{a b}(\underline{x})\right)=\left(\overline{u^{2}} / u(t)^{2}\right)<\epsilon\left(\underline{x}, h_{a b}(\underline{x}), t\right)>
$$

where $\langle\cdot\rangle$ represents the bathymetric classification and averaging procedure. This model was used with the map of bottom bathymetry to produce gridded time-mean dissipation estimates.

\subsection{The Inverse Model}

\subsubsection{Model Equations}

The abyssal regime is the focus for the circulation problem considered here. In this regime, we make the a priori assumption that the diabatic terms in the governing equations are significant. All the dynamical equations will be cast in terms of flow along isopycnals and flow across isopycnals. The dynamically relevant density variable for this analysis is neutral density $\left(\sigma_{n}\right.$, Jackett and McDougall, 1996). The neutral density coordinates are everywhere parallel to the locally defined potential density. Neutral density values have resemblance to potential density referenced to the sea surface, and the dimensions of $\mathrm{kg} \mathrm{m}^{-3}$ will generally be discarded in text that follows. Preliminary examination of multiple year hydrographic data revealed that the temporal variation of the isopycnal-height field was negligible (Ledwell et al., 1999). Therefore, we will assume that much of the flow regime is in steady geostrophic-balance.

The flow regime is divided into into a series of density layers, each characterized by a layer thickness $h(x, y)$. In these layers, geostrophic advection is related to 
isobaric gradients of geopotential anomaly $(\varphi)$ by

$$
\underline{u}=\hat{z} \times \frac{\nabla \varphi}{f}+\underline{u_{0}},
$$

where $\underline{u}_{0}$ is a reference level velocity. The diapycnal advection can be expressed in terms of the divergence of dissipated energy,

$$
w_{*} N^{2}=\Gamma \frac{\partial \bar{\epsilon}}{\partial z}
$$

where $\Gamma=R_{f}\left(1-R_{f}\right)^{-1}=\left(-J_{b} / \bar{\epsilon}\right)$ is the ratio of buoyancy flux to dissipation. The parameter $\Gamma$ is assumed to be in the range $0.20 \pm 0.04$, an assumption that breaks down under conditions of very weak stratification when the buoyancy flux $J_{b}$ approaches zero. Thus, the advection of planetary vorticity through a density layer, as previously stated in (3.1), is given by

$$
\beta v=\frac{f}{h} \underline{u} \cdot \nabla h+\frac{f}{h}\left(w_{*}\left(z_{u}\right)-w_{*}\left(z_{l}\right)\right),
$$

or, through application of (3.8) and rearranging terms,

$$
\frac{h_{x}}{h} u_{0}+\left(\frac{h_{y}}{h}-\frac{\beta}{f}\right) v_{0}+\frac{1}{h}\left(w_{*}\left(z_{u}\right)-w_{*}\left(z_{l}\right)\right)=-u_{r} \frac{h_{x}}{h}-v_{r}\left(\frac{h_{y}}{h}-\frac{\beta}{f}\right),
$$

where $\underline{u_{r}}$ is the relative geostrophic velocity given by $\underline{u}-\underline{u_{0}}$ in (3.8). Thus, the problem of determining the lateral flow $\underline{u}$ is reduced to determining the "level of no motion" for the vertical integration constants $\left(u_{0}, v_{0}\right)$ of a thermal-wind balance.

In layers where flow encounters topography, the use of geostrophic balance is questionable. A modified momentum balance, such as that of the bottom Ekman layer, may be called for in regions where a density layer comes within $O(100 \mathrm{~m})$ of the bottom. Additionally, the difficulty of defining the geopotential anomaly along pressure surfaces interrupted by bathymetry severely limits the practical use of (3.8) and (3.10) in deep layers. For these reasons, it is necessary to employ additional dynamical constraints on the flow. This is achieved through the use of integrated advective budgets for mass, 


$$
\iint d y d z\left(\left.u\right|_{x} ^{x+\gamma}\right)+\iint d x d z\left(\left.v\right|_{y} ^{y+\Delta}\right)+\iint d x d y\left(\left.w_{*}\right|_{z_{l}} ^{z_{u}}\right)=0
$$

and for potential temperature

$$
\begin{gathered}
\iint d y d z\left(\left.u \Theta^{\prime}\right|_{x} ^{x+\gamma}\right)+\iint d y d z\left(\left.v \Theta^{\prime}\right|_{y} ^{y+\Delta}\right)+\iint d x d y\left(\left.w_{*} \Theta^{\prime}\right|_{z_{l}} ^{z_{u}}\right)= \\
\iint d y d z\left(\left.\kappa \Theta_{x}\right|_{x} ^{x+\gamma}\right)+\iint d y d z\left(\left.\kappa \Theta_{y}\right|_{y} ^{y+\Delta}\right)+\iint d x d y\left(\left.k \Theta_{z}\right|_{z_{l}} ^{z_{u}}\right)
\end{gathered}
$$

The integrated expressions (3.12) and (3.13) are bounded in a control volume of meridional and zonal extent $(\gamma, \delta)$, and vertically bounded in a density layer by the surfaces $z_{u}(x, y)$ and $z_{l}(x, y)$. In the temperature advection equation, $\Theta^{\prime}$ is the temperature anomaly relative to the mean temperature in each cell. The vertical diffusivity $k$ is assigned using the modeled dissipation data with the Osborn (1980) relation

$$
k=\left(\Gamma / N^{2}\right) \bar{\epsilon}
$$

In addition to the assumption concerning the value of $\Gamma$ discussed for (3.9), this diffusivity relation assumes that the buoyancy flux can be expressed in the Fickian form $J_{b}=-k N^{2}$. A Fickian form is also used to express the lateral flux terms, where the isopycnal diffusivity $\kappa$ is an unconstrained parameter which will be treated as an unknown.

The advective budgets assume nothing about the nature of the momentum balance, and for this reason their applicability is not limited to conditions of geostrophic balance. However, without any model for the momentum balance, the components of the lateral flow in (3.12) and (3.13) must be treated as unknowns at all depths, whereas (3.11) relates the velocity at all depths to the single component pair $\left(u_{0}, v_{0}\right)$.

\subsubsection{Treatment of Data}

The above dynamics can be employed in an inverse model where the observed density field $\left(h(x, y), N^{2}\right)$, geopotential anomaly field $\varphi(x, y, p)$ and temperature 
field $\Theta(x, y, z)$ are used to solve for the unknowns $\left(\underline{u}, w_{*}, \kappa\right)$. The geometry of the inversion grid needs to accommodate both the finite-element nature of the betaspiral vorticity balance (3.11) and control-volume cells for (3.12) and (3.13). This is done using a staggered grid of nested cells, as is shown in figure 3.6. For each density layer, three lateral planes define the cell; a central plane along which the lateral velocity components are constrained and upper and lower planes on which the diapycnal velocities are constrained.

The 1997 HRP survey data were used to provide the observed hydrographic fields. The temperature and salinity data were first used to calculate the geopotential anomaly field on isobaric surfaces. These fields were modeled using a polynomial fit of the form

$$
\varphi(X, Y)=\phi_{0}+\phi_{x} X+\phi_{y} Y+\phi_{x x} X^{2}+\phi_{x y} X Y+\phi_{y y} Y^{2},
$$

where $(X, Y)$ are the Cartesian coordinates centered on the survey and $\phi_{(\cdot)}$ are the coefficients of the fit. The gradient of the fit is used with (3.8) to give estimates of the geostrophic flow relative to $2000 \mathrm{~m}$. This reference level was chosen based on ideas about basin-scale water masses, with $2000 \mathrm{~m}$ being roughly between northward flowing intermediate water (referred to as Circumpolar Water by Zhang and Hogg, 1992) and southward flowing North Atlantic Deep Water (NADW). The hydrographic data were sorted into layers of neutral density, and fits of type (3.15) were used to give quasianalytic expressions for $\Theta(x, y), \Theta_{z}(x, y), h(x, y)$ and $N^{2}(x, y)$ in each layer. This layered system comprises the static stratification field which is used for the inversion. Assignments of geostrophic shear for each layer were made through explicit reference to the isobaric surfaces used in the geopotential anomaly calculation. Thus, the inverse domain is a layered system of hydrographic estimates on a staggered grid of nested-cells. Various domain dimensions were explored, but the calculations discussed here cover $\left(14^{\circ} \mathrm{W}-24^{\circ} \mathrm{W}\right.$, $19^{\circ} \mathrm{S}-25^{\circ} \mathrm{S}$ ) with 6 density layers, bounded above by $\sigma_{n}=27.6$ (the $1000 \mathrm{~m}$ average depth potential-density surface) and below by the sea-floor bathymetry. Each cell of the staggered grid has Mercator dimensions of $\left(0.5^{\circ}\right)^{2}$, and the altimetry-derived bathymetry data of Smith and Sandwell (1997) were subsampled onto this grid. The modeled dissipation data (3.7) were incorporated into the inverse domain by identifying each estimation point as a bathymetric crest, slope, or canyon. This 


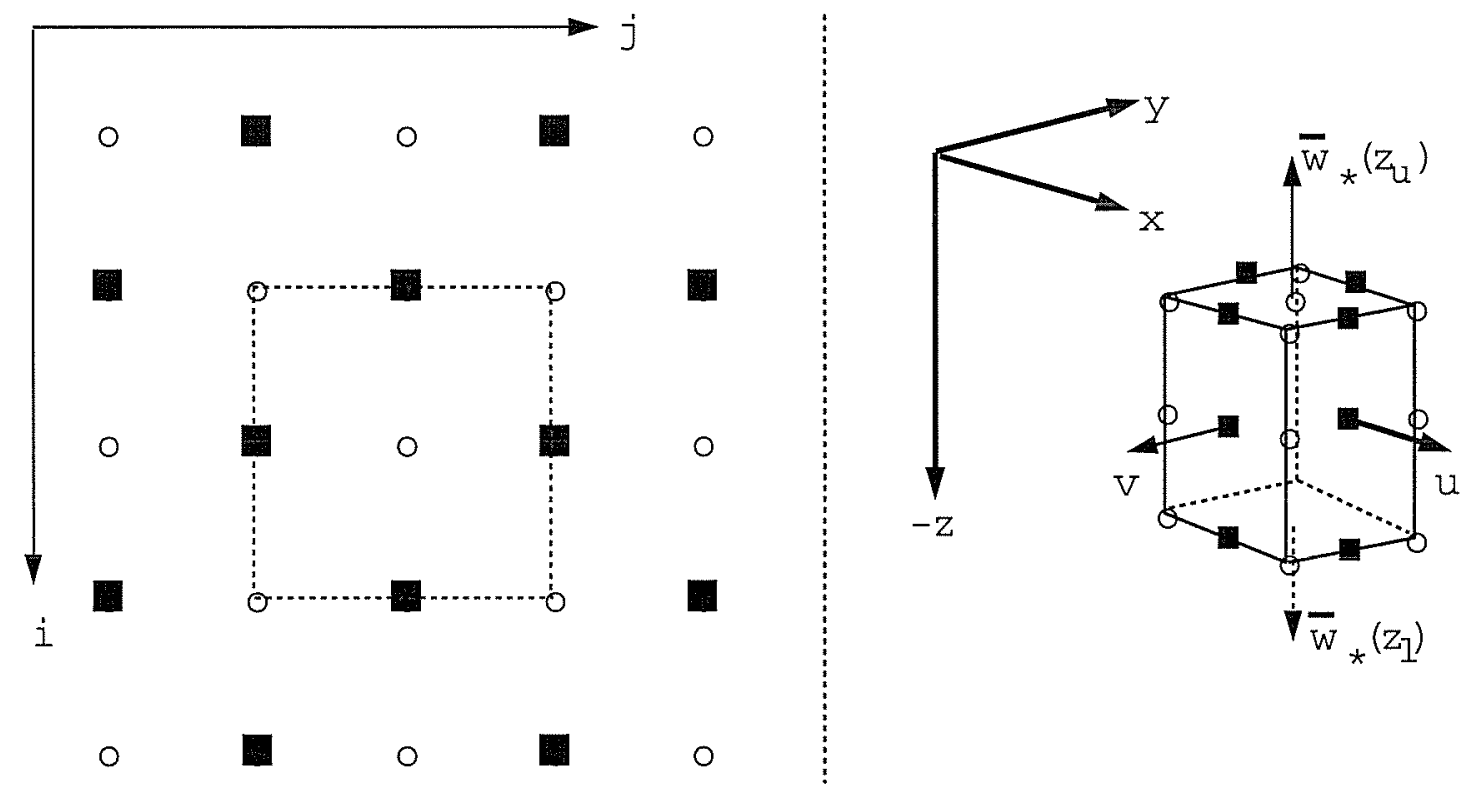

Figure 3.6: Geometry of the staggered grid of nested cells used to accommodated both finite element and volume integrated inverse calculations. Estimation points (squares) participate in up to 4 cells. Each density layer is composed of 3 lateral planes (right panel). Lateral flow is constrained on the central plane, while upper and lower planes constrain the diapycnal advection. 
was done through a classification scheme that utilizes both the first and second zonal-derivatives of the bathymetric topography which were computed at 2 arc minute resolution.

Figure 3.7 shows a projection of the subsampled bathymetry underlying the plotted estimation grid. The system of FZs is very coarsely resolved. A zonal section showing the modeled layers deeper than $2500 \mathrm{~m}$ along $22^{\circ} \mathrm{S}$ is presented in figure 3.8, where the fitted density field is compared with the observed field. Residuals between the fitted and observed density field are typically $20 \mathrm{~m}$ to $50 \mathrm{~m}$. Correlations exist in these residuals along the survey track, and the groups of 3 stations were sampled during a single phase of the diurnal tide. Thus, the low order of the fitted polynomials was implemented as a way of dealiasing the tidal noise in the density field. The residuals of the fitted density field lack correlated structure on the larger scales which encompass multiple survey sections.

The low order of the polynomial fits used to condition the hydrographic data provide an effective means of removing ageostrophic noise, such as tidal displacements of the isopycnal height field. Such smoothing generally removes all small spatial-scale features in the data. For example, flow patterns trapped near ridge topography may account for significant transport in the abyss where large-scale flow patterns are weak. Evidence for such features is not present in the observations, but this may be a consequence of coarse sampling. The use of a low-order spatial filter assumes that the governing dynamics are quasi-geostrophic in nature, with flow structures with spatial scales less than $\mathrm{O}\left(R_{d}\right)$ being insignificant at first order in the scaled governing equations.

At scales larger than $\mathrm{O}\left(R_{d}\right)$, an assessment of hydrographic noise was done using Monte Carlo methods. In particular, the influence of $50 \mathrm{~m}$ amplitude verticaldisplacements of the isopycnal-height field arising from tidal frequency motions was examined. To accomplish this, the observed isopycnal height-field was perturbed by a random field of Gaussian noise with $50 \mathrm{~m}$ standard deviation. The perturbed density field was then treated with the polynomial smoothing discussed above, and this smoothed but perturbed hydrography was used for the inversion. In this manner, 10 alternate realizations of the density field were produced, and an ensemble of inverse solutions was calculated. 

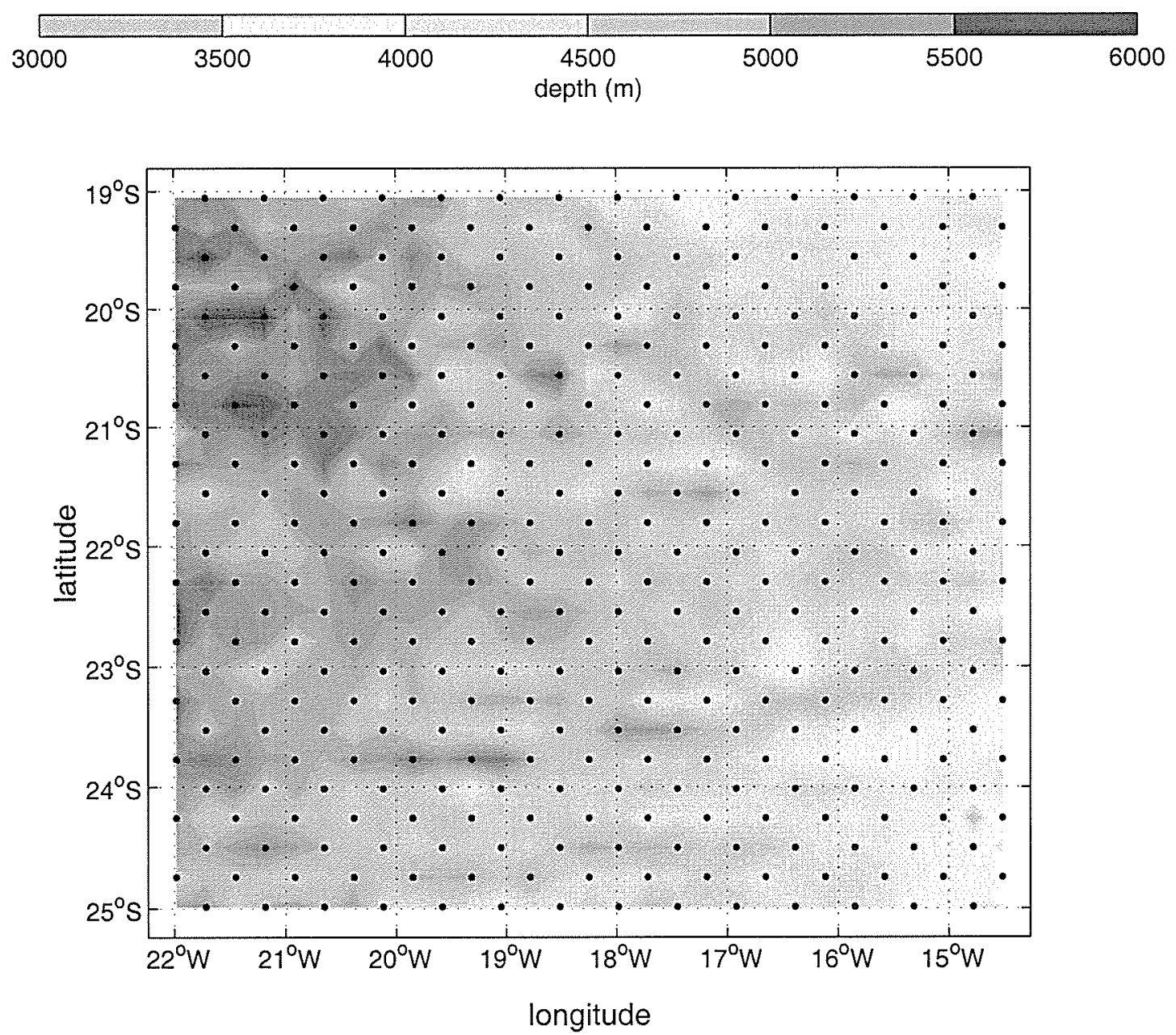

Figure 3.7: Map of the inversion domain. The staggered estimation grid is shown with the $0.25^{\circ}$ subsampled bathymetry. Hydrographic data were mapped onto this grid using polynomial fits. Modeled profiles of $\bar{\epsilon}\left(h_{a b}\right)$ were assigned to each grid point according to bathymetric classification by first and second derivatives of the bottom topography. 


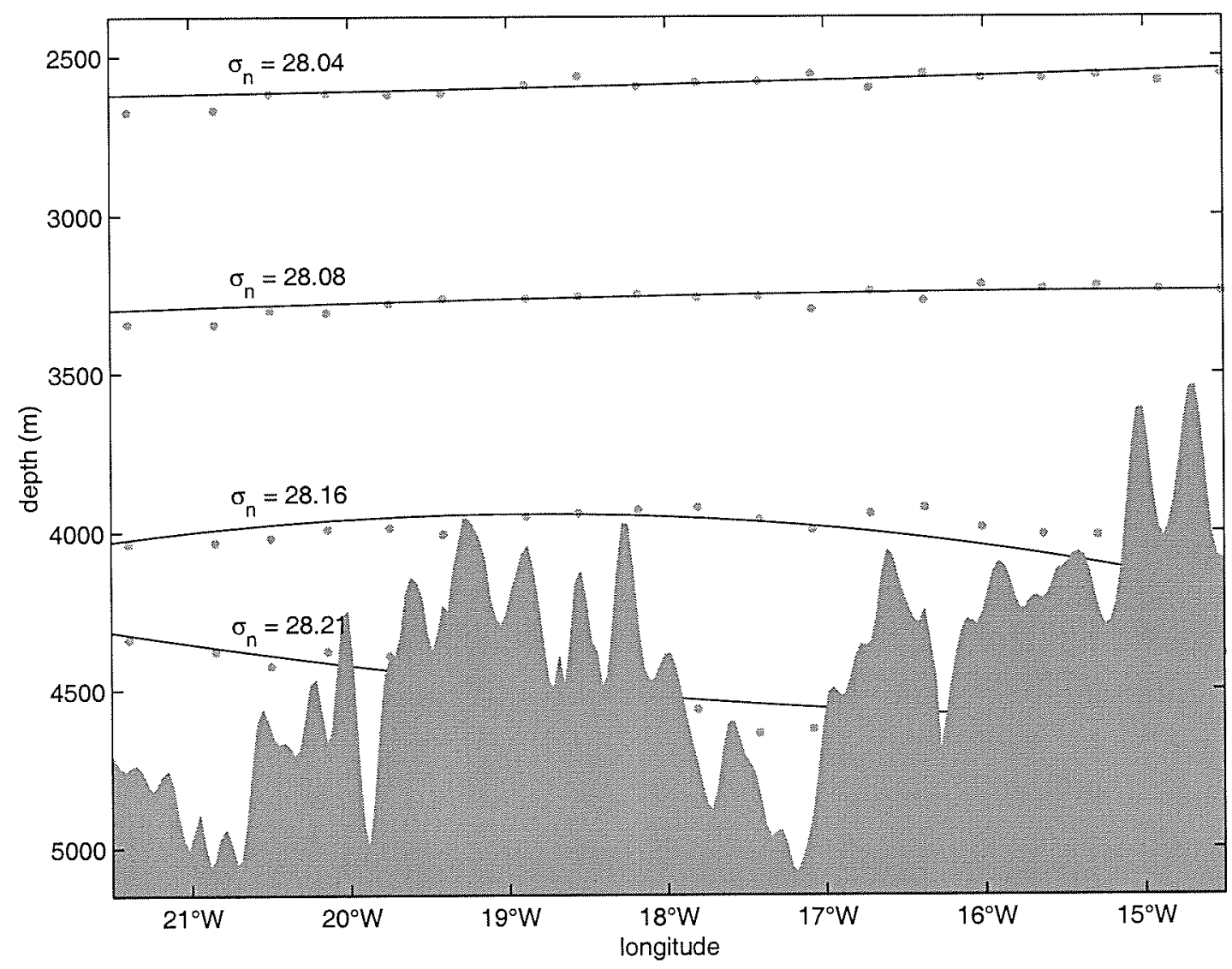

Figure 3.8: A section comparing the the observed and fitted isopycnal-height field along $22^{\circ} \mathrm{S}$. Correlations of 3 station groups (spatial scales of $100 \mathrm{~km}$ ) exist in the residuals of the fits, and this is associated with tidal displacements of the isopycnal height field. Isopycnals that define the 4 deepest density layers are shown, and these are the surfaces used for estimating the diapycnal advection. The target isopycnal for the tracer release was $\sigma_{n}=28.16,\left(\sigma_{4}=45.98, \theta=0.8^{\circ}\right)$. 


\subsubsection{Linear Algebraic Equations}

The fitted hydrographic field and associated dissipation field were then used to assemble a linear system of coupled equations of the form

$$
\underline{\underline{A} x}+\underline{n}=\underline{d},
$$

where $\underline{\underline{A}}$ is the coefficient matrix of the terms in (3.11-3.13) involving the unknowns $\underline{x}=\left[\underline{u_{0}}, \underline{v_{0}}, \underline{w_{*}}, \underline{\kappa}\right]^{T}$, and $\underline{d}$ is the vector of observable terms. The vector $\underline{n}$ explicitly denotes the residuals of the inverse model fit, $\underline{n}=\underline{d}-\underline{\underline{A}} \underline{x}$. To this linear system of equations, we add a set of constraints for the diapycnal advection

$$
\underline{\underline{I}}\left[\left(\Gamma / N^{2}\right) \overline{\epsilon_{z}}\right]=\underline{w_{\epsilon}},
$$

where the $(\epsilon)$ subscript is used to denote that $\underline{w_{\epsilon}}$ is the vector of a priori estimates, and not the estimate $w_{*}$ to be determined through inversion. The linear equations (3.16) and (3.17) can easily be incorporated into a single linear expression

$$
\underline{\underline{E x}}+\underline{n}=\underline{y}
$$

which combines the model equations with the constraints. This linear system is solvable by least-square methods that generally minimize the residuals of the model fit. A variety of inversion procedures are discussed by Wunsch (1996), and the Gauss-Markov estimator was chosen for the calculations needed here. This form of inversion produces an estimate of $\underline{x}$ which utilizes a priori knowledge of the statistics for $\underline{x}$ and $\underline{n}$. This is done through a specification of the covariance matrices. The covariance matrix of unknowns is taken to be the diagonal matrix composed of the variance quantities $\left.<(u-\bar{u})^{2}\right\rangle \sim\left(0.1 \mathrm{~cm} \mathrm{~s}^{-1}\right)^{2}$, $<\left(w_{*}-\overline{w_{*}}\right)^{2}>\sim\left(1 \mathrm{~m} \mathrm{yr}^{-1}\right)^{2}$, and $<(\kappa-\bar{\kappa})^{2}>\sim\left(100 \mathrm{~m}^{2} \mathrm{~s}^{-1}\right)^{2}$. The assumed scale for the lateral flow is justified by float observations (Hogg and Owens, 1999), while the variance estimate for the diapycnal flow comes directly from the statistics of the dissipation constraint (3.17). A scale for the lateral diffusivity was derived from a mixing length estimate. Using an eddy kinetic-energy of $1 \mathrm{~cm}^{2} \mathrm{~s}^{-2}$ (Hogg and Owens, 1999) and a deformation radius length-scale of $10 \mathrm{~km}$ suggests $u^{\prime} l^{\prime} \sim 100 \mathrm{~m}^{2} \mathrm{~s}^{-1}$. A diagonal covariance matrix for the model residuals 
was constructed using an uncertainty analysis of the elements of $\underline{y}$. For example, the scale for the residuals of the beta-spiral equations involves the error propagation of terms like $(\delta h)^{2},\left(\delta h_{x}\right)^{2}$ and $\left(\delta h_{y}\right)^{2}$ in $(3.11)$. The $\delta(\cdot)$ terms are calculated using the standard error of the modeled hydrography, and the expressions for error propagation follow from standard techniques (Bevington and Robinson, 1992). Typical signal to noise ratios for the elements of the observed terms of the geostrophic model equations, are $(|y| / \delta y) \sim(1 / 5)$ for the beta-spiral and heat equations and $(\underline{|y|} / \delta y) \sim 1$ for the mass and diapycnal advection constraints. The statistical uncertainties assigned to the ageostrophic advective budgets are $\mathrm{O}\left(0.01 \times 10^{6} \mathrm{~m}^{3} \mathrm{~s}^{-1}\right)$ and $\mathrm{O}\left(1 \times 10^{2} \mathrm{~m}^{3 \circ} \mathrm{C} \mathrm{s}^{-1}\right)$ for the mass and heat respectively.

In addition to the statistical scalings provided by the two covariance matrices, several nonstatistical scalings are used to improve the rank of the inversion. The linear model (3.18) is nondimensionalized by column scaling with a diagonal matrix of dimensional scales $\underline{\underline{D}}$ so that all the elements of $\underline{x}$ are $\mathrm{O}(1)$. Additionally, the rows of $\underline{\underline{E}}$ are scaled by the equation norms $\operatorname{diag}(\underline{\underline{N}})$ and the density layer temperature-contrasts $\operatorname{diag}(\underline{\underline{T}})$ associated with the thermal advection equations. The later scaling accounts for the general decrease in temperature contrast as layers get deeper and is discussed by Zhang and Hogg (1992). Thus, the Gauss-Markov estimate for the nondimensional variables $\underline{x^{\prime}}$ and the associated uncertainty $\underline{\delta x^{\prime}}$ is given by

$$
\begin{aligned}
\underline{x^{\prime}} & =i n v\left(\underline{\underline{C_{x x}^{\prime}}}+\underline{\underline{E^{\prime T}}} \underline{\underline{C_{n n}^{\prime}}}{ }^{-1} \underline{E^{\prime}}\right) \underline{E^{\prime T}} \underline{\underline{C_{n n}^{\prime}}}{ }^{-1} \underline{y^{\prime}} \\
\underline{\delta x^{\prime}} & =i n v\left(\underline{\underline{C_{x x}^{\prime}}}+\underline{\underline{E^{\prime T}}} \underline{\underline{C_{n n}^{\prime}}}{ }^{-1} \underline{\underline{E^{\prime}}}\right)
\end{aligned}
$$

where the scaled matrices are $\underline{\underline{E^{\prime}}}=\underline{\underline{N}}^{-1} \underline{\underline{T^{-1}}} \underline{\underline{E D}}, \underline{\underline{C_{x x}^{\prime}}}=\underline{\underline{D}}^{-2} \underline{\underline{C_{x x}}}$, $\underline{\underline{C_{n n}^{\prime}}}=\underline{\underline{N}}^{-2} \underline{\underline{T}}^{-2} \underline{\underline{C_{n n}}}$ and $\underline{y^{\prime}}=\underline{\underline{N}}^{-1} \underline{\underline{T}}^{-1} \underline{y}$.

\subsubsection{Results}

The matrix model for the 6 layer system consisted of about 7000 equations for 3200 unknowns. The Singular Value Decomposition (SVD) of the scaled matrix equation (3.18) was calculated, and the parameter and data resolution matrices of the SVD were examined (discussed by Wunsch 1996). While parameters for lateral flow and diapycnal advection were well resolved, the lateral diffusivity $\kappa$ 
was poorly resolved at all levels. In a series of initial calculations, various assumptions about the lateral diffusivity were applied to the model. These included the use of a constant diffusivity on each density layer, as well as a depth independent lateral diffusivity. In all cases, estimates for $\kappa$ were near zero, with $\mathrm{O}\left(100 \mathrm{~m}^{2} \mathrm{~s}^{-1}\right)$ uncertainties. Attempts to constrain $\kappa$ to values greater than zero lead to in increase in model residuals. Additionally, diagnostic scaling of the thermal advection equations indicated that a primary balance occurs between the term involving advection (both lateral and vertical) and the vertical diffusion term. For these reasons, terms involving the lateral diffusivity were dropped from the thermal advection equations, leaving the three dimensional flow field $\left(\underline{u}, w_{*}\right)$ as the model unknowns.

The matrix of data eigenvectors $\underline{\underline{U}}$ of the model matrix were used to calculate the data resolution matrix $\underline{\underline{U}}^{T} \underline{\underline{U}}$, and this was used to diagnose the relative importance of the model equations. The rank of each equation is a number between zero and unity given by the diagonal elements of the data resolution matrix, as shown in figure (3.9). Equations that contribute independent information to the estimation problem have data resolution near unity. Lesser values of data resolution indicated the reduced influence of an equation in the inversion. Structure in the data resolution matrix is associated with the geometry of the model matrix. Generally, groups of equations within the same density layer are apparent by the modulated structure of $\operatorname{diag}\left(\underline{\underline{U}}^{T} \underline{\underline{U}}\right)$. In deep layers, $\beta$-spiral (layers 4 and the western part of layer 5), mass and thermal advection equations contribute strongly to the estimate of flow, with constraints on $w_{*}$ contributing to a lesser extent. However, the role of microstructure information in the deep layers is not easily dismissed, as the vertical diffusion term of the thermal advection balance also relies on the dissipation data.

Following inversion, model residuals were examined. In general, the model residuals were distributed about zero without coherent structure, with meansquare magnitudes in good agreement with the a priori assumed scales in $\operatorname{diag}\left(\underline{\underline{C_{n n}}}\right)$. For clarity, the shallow and deep regimes of the inverse solution are discussed separately. 


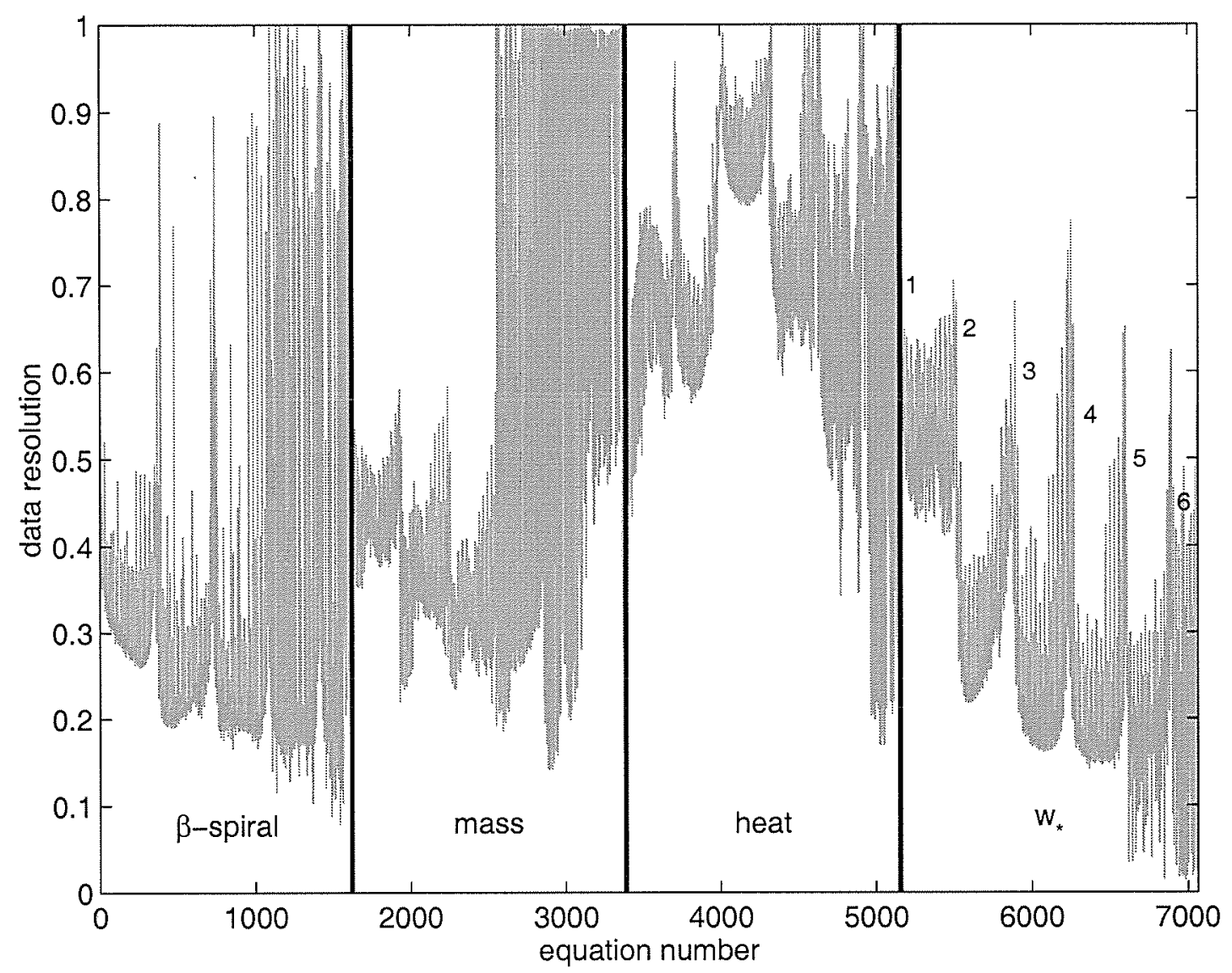

Figure 3.9: The diagonal elements of the data resolution matrix. The four classes of model equations are ordered by equation number. For each equation class, increasing equation numbers correspond to deeper layers of the inverse geometry, and groups of equations for a particular layer are associated with the modulated structure of the data resolution matrix. Values of data resolution near unity indicate that equations are contributing independent information to the inversion, while lesser values signify a reduction in an equation's individual influence on the solution. $\beta$-spiral, mass and heat (thermal advection) equations all contribute strongly to estimates of deep flow. The influence of the diapycnal advection constraints is reduced in successively deeper layers, as numbered 1-6. 


\section{Shallow Flow}

Diapycnal mixing in the layers shallower than $3000 \mathrm{~m}$ was weak, with diffusivity estimates $k=\left(\Gamma / N^{2}\right) \bar{\epsilon}<1 \mathrm{~cm}^{2} \mathrm{~s}^{-1}$ Consequently, the diapycnal advection through these layers was also weak, with $\left|w_{*}\right|<1 \mathrm{~m} \mathrm{yr}^{-1}$. Diabatic forcing of this magnitude plays a rather insignificant role in the determination of the lateral flow, as the diabatic term in (3.11) is typically less than $10 \%$ of the adiabatic stretching term represented by $\underline{u} \cdot(\nabla h / h)$. Therefore, the weak diabatic flow in these shallow layers in not further discussed.

Within the domain treated by the inversion (west of $14^{\circ} \mathrm{W}$ ), the bottom topography does not shoal less than $3000 \mathrm{~m}$ with the exception of a seamount in the southeast corner of the domain. Thus, geostrophic dynamics were assumed valid everywhere, and the combined usage of the beta spiral with mass and temperature advection yielded an overdetermined system for the barotropic flow at each grid point. The estimated geostrophic flow fields (relative plus reference level) for the 3 layers shallower than $3000 \mathrm{~m}$ are shown in figures 3.10, 3.11 and 3.12 . The largest current speeds in these layers are $2 \mathrm{~mm} \mathrm{~s}^{-1}$, and the mean speeds are around $0.5 \mathrm{~mm} \mathrm{~s}^{-1}$. The flows in these layers are generally consistent with ideas about water mass circulation. Above and below a level of very weak flow $\left(\sigma_{n}=27.83,2200 \mathrm{~m}\right)$, the intermediate water layer $\left(\sigma_{n}=28.01,1400 \mathrm{~m}\right)$ flows in a northward direction, while the NADW layer $\left(\sigma_{n}=28.06,2900 \mathrm{~m}\right)$ flows in a southward direction. The local nature of these flow domains make detailed comparisons with previous results difficult, as most past work has considered basin scales. However, the flows found here have qualitative similarity to flow estimates discussed by Zhang and Hogg (1992).

An ensemble of 10 velocity estimates are shown at each grid point. The spread of vectors at each point is the result of uncertainty in the determination of the reference level flow due to $\pm 50 \mathrm{~m}$ noise in the isopycnal-height field. The flow estimates are most sensitive in the eastern edge of the domain, where fewer stations were occupied. This sensitivity amounts to an uncertainty in flow direction not greater than $\pi / 12$ radians. Statistical uncertainty in the magnitude of the flow field is typically (0.1-0.2) $\mathrm{mm} \mathrm{s}^{-1}$, as assessed from standard error propagation techniques used with the polynomial fits to the geopotential-anomaly fields. 


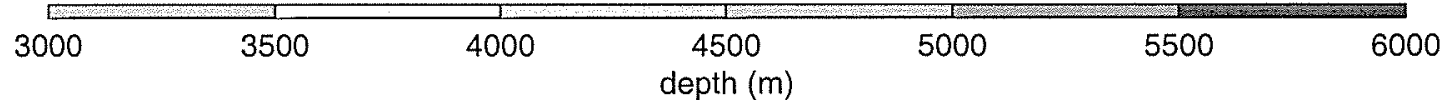

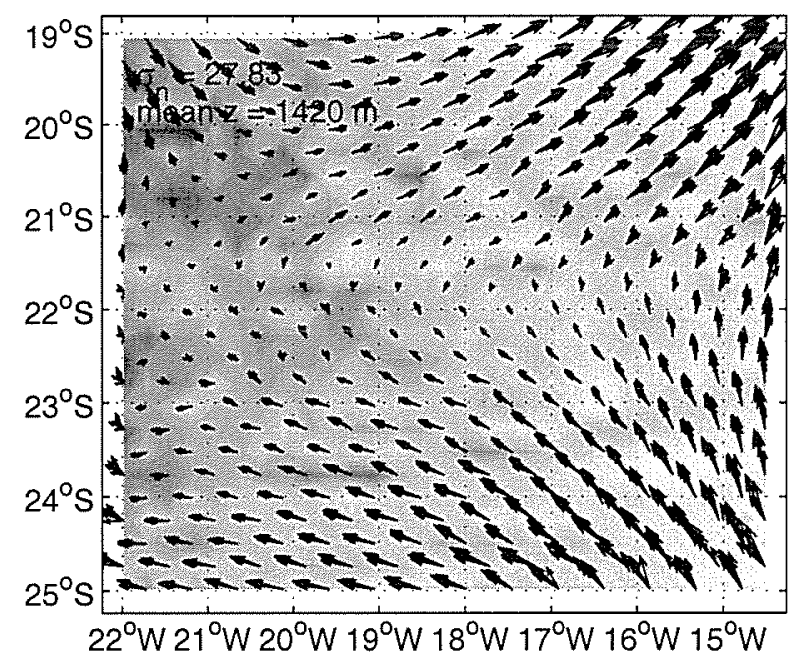

Figure 3.10: Layer-averaged geostrophic flow (relative plus reference level) of the density layer $27.60<\sigma_{n}<27.97$. This density class is typically referred to as intermediate water, and the largest vectors correspond to speeds of $2 \mathrm{~mm} \mathrm{~s}^{-1}$. At each estimation point 10 alternate estimates are shown from the Monte Carlo set of $50 \mathrm{~m}$ perturbations to the isopycnal-height field. The central isopycnal-level and the mean layer-depth are reported in the corner of the figure. 


\begin{tabular}{|c|c|c|c|c|c|}
\hline 3000 & 3500 & 4000 & $\begin{array}{c}4500 \\
\text { depth }(m)\end{array}$ & 5000 & 5500 \\
\hline
\end{tabular}

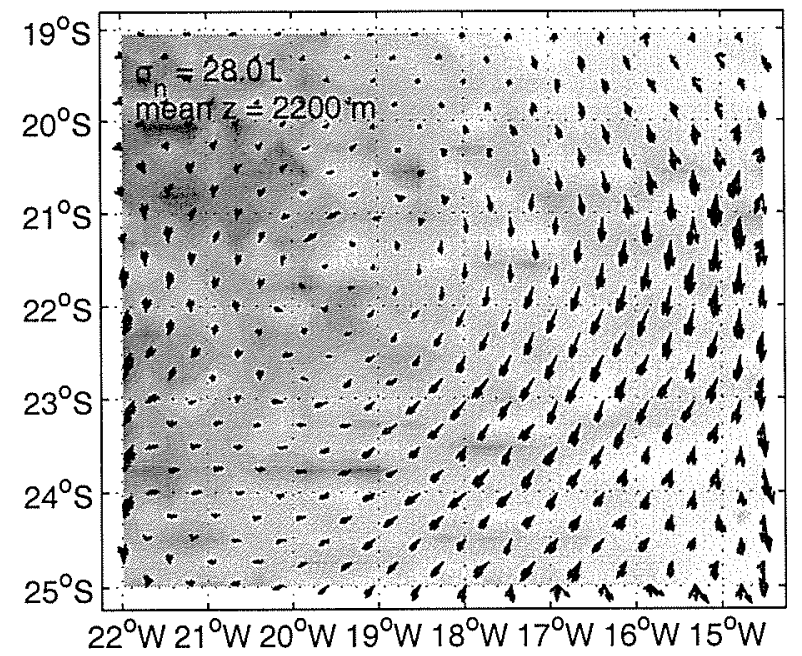

Figure 3.11: Layer-averaged flow of the density layer $27.97<\sigma_{n}<28.04$. The largest vectors correspond to speeds of $1 \mathrm{~mm} \mathrm{~s}^{-1}$. 


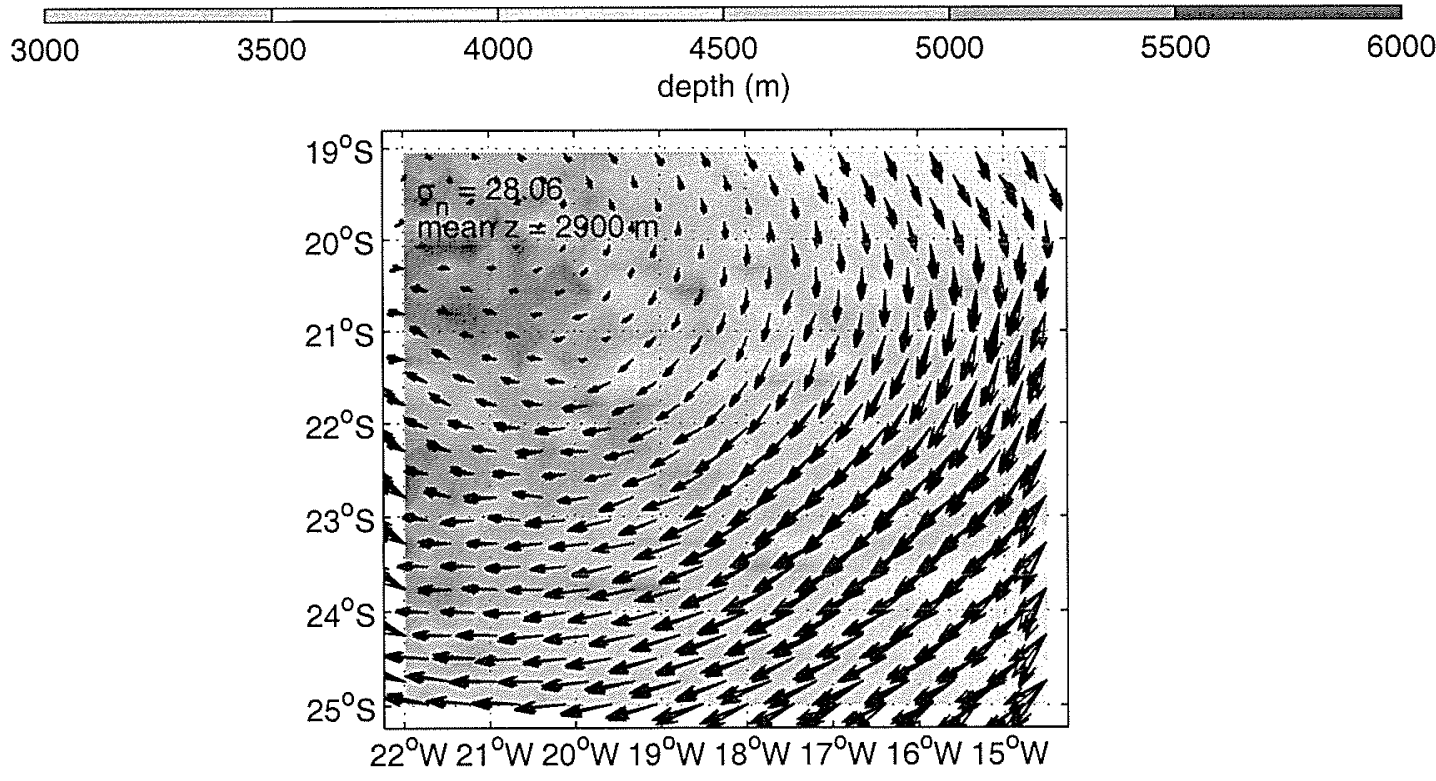

Figure 3.12: Layer-averaged flow of the density layer $28.04<\sigma_{n}<28.08$. This density class is associated with NADW, and the largest vectors correspond to speeds of $2 \mathrm{~mm} \mathrm{~s}^{-1}$. 


\section{Deep Flow}

The estimate of flow at deeper levels is more central to the theme of the tracer release experiment. In the layers deeper than $3000 \mathrm{~m}$, bottom bathymetry has a clear influence on the flow dynamics. In particular, proximity to the bottom is the primary issue related to the magnitude of the diabatic forcing. The vertical diffusivity characterizing these layers is $\mathrm{O}\left(1 \mathrm{~cm} \mathrm{~s}^{-1}\right)$ or greater, and this corresponds to an increased importance of the diabatic terms in governing equations. The estimated lateral flow fields, with associated vertical diffusivities, are shown in figures $3.13,3.14$ and 3.15. Flow in these layers is generally southward and westward, with average current magnitudes of $(2-4) \mathrm{mm} \mathrm{s}^{-1}$, with the faster currents occurring in the western portion of the domain exceeding $0.5 \mathrm{~cm} \mathrm{~s}^{-1}$. The influence of $\pm 50 \mathrm{~m}$ noise in the isopycnal-height field has also been evaluated, and the lateral flow is very stable to this type of perturbation.

The neutral density range $28.12<\sigma_{n}<28.18\left(45.88<\sigma_{4}<45.96\right.$ is the potential density referenced to $4000 \mathrm{dbar}$ ) is of particular interest, because this density layer bounds the target isopycnal used for the tracer release, $\sigma_{n}=$ $28.16\left(\sigma_{4}=45.94\right)$. The tracer inventory made during the 1997 survey indicated that the tracer's center of mass was advected $130 \mathrm{~km}$ southwest over a period of 14 months, as reported by Toole et al. (1997b). This is equivalent to a time average speed of $3.6 \mathrm{~mm} \mathrm{~s}^{-1}$. The average velocity in the density layer $28.12<\sigma_{n}<28.18$, as estimated by the inversion for the region $\left(18.5^{\circ} \mathrm{W}-22^{\circ} \mathrm{W}\right.$, $\left.22^{\circ} \mathrm{S}-25^{\circ} \mathrm{S}\right)$, is $|\underline{u}|=(3 \pm 0.3) \mathrm{mm} \mathrm{s}^{-1}$ in the direction $220^{\circ}-230^{\circ}$. Thus, the lateral flow estimate given by the inversion is consistent with the rate of lateral advection measured by the tracer. The calculated flow in these layers is also consistent with float observations made in this region during 1994-1996, as reported by Hogg and Owens (1999).

A detailed discussion of tracer- and microstructure derived diffusivities for the target isopycnal is given by Ledwell et al. (1999). The tracer derived result of $3 \mathrm{~cm}^{2} \mathrm{~s}^{-1}$ compares well with the $95 \%$ confidence interval of the microstructure estimate, $(2.3 \pm 1) \mathrm{cm}^{2} \mathrm{~s}^{-1}$. The later estimate was made using the 14 month tracer concentration field as weighting in the spatial average, and is thus not representative of the larger diffusivities of $(5-10) \mathrm{cm}^{2} \mathrm{~s}^{-1}$ that occur east of the tracer patch. As is apparent in figures 3.14 and 3.15 , there is an order of magnitude 


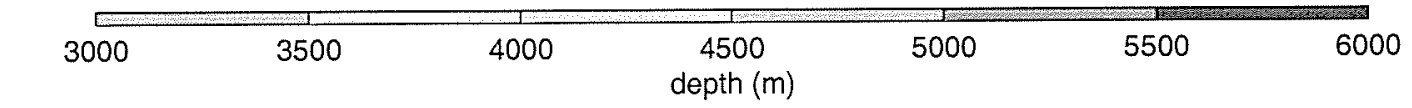
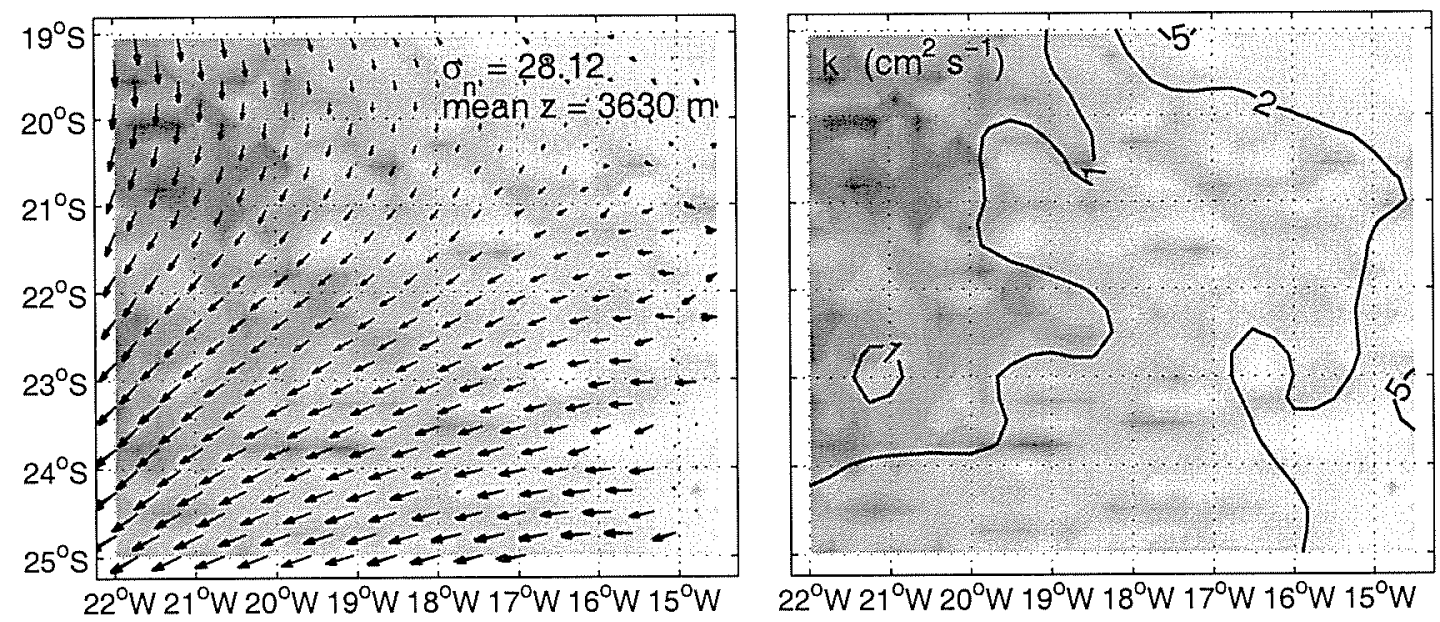

Figure 3.13: Layer-averaged flow (left panel) and diapycnal diffusivities (right panel) for the density layer $28.08<\sigma_{n}<28.16\left(45.83<\sigma_{4}<45.94\right)$. Geostrophic balance was assumed at grid points whose lateral distance to bathymetric peaks exceeded $15 \mathrm{~km}$ and $h_{a b}>100 \mathrm{~m}$. The ensemble of 10 Monte Carlo assessments of $50 \mathrm{~m}$ perturbations to the isopycnal-height field are shown, but the various solutions are nearly indistinguishable. The average flow speed is $3 \mathrm{~mm} \mathrm{~s}^{-1}$. Turbulent diffusivities are shown for the central surface $\sigma_{4}=28.12$. 


\begin{tabular}{|c|c|c|c|c|c|}
\hline 3000 & 3500 & 4000 & $\begin{array}{c}4500 \\
\text { depth (m) }\end{array}$ & 5000 & 5500 \\
\hline
\end{tabular}
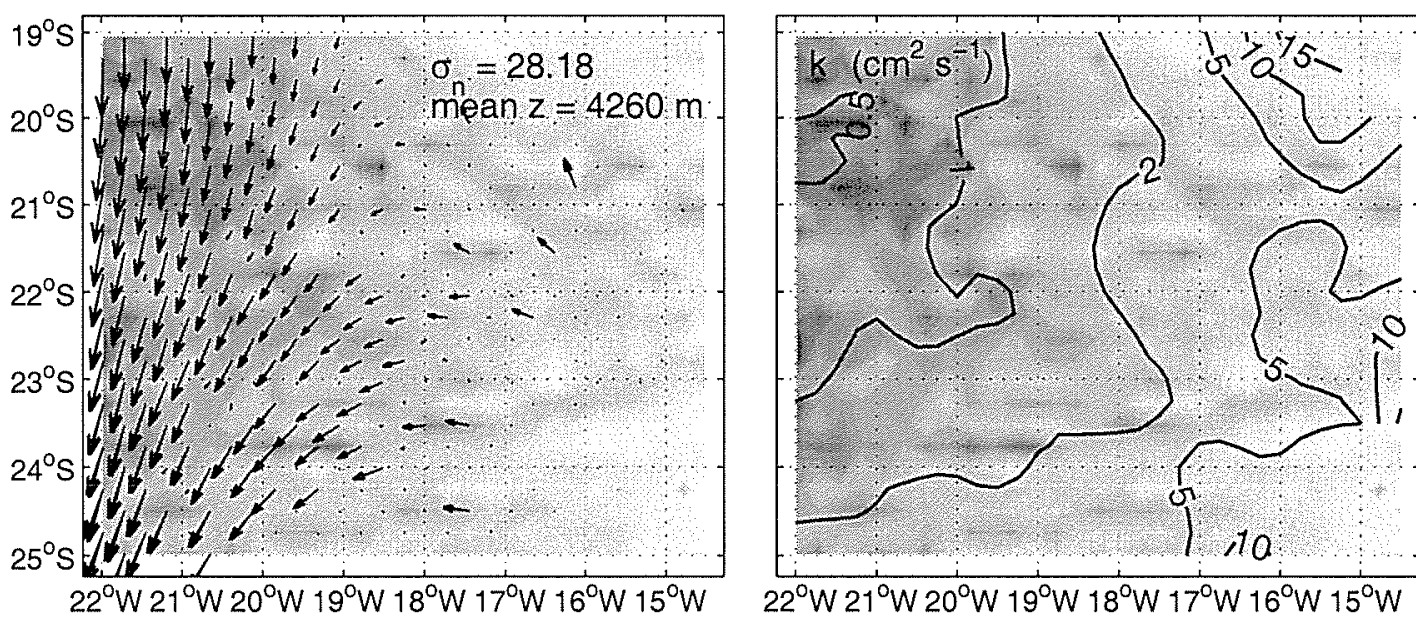

Figure 3.14: Layer-averaged flow and diapycnal diffusivities for the density layer $28.16<\sigma_{n}<28.21\left(45.94<\sigma_{4}<45.99\right)$. Southward and westward flow characterizes the western half of the domain with speeds (4-6) $\mathrm{mm} \mathrm{s}^{-1}$. Turbulent diffusivities are shown for the central surface $\sigma_{4}=28.18$, and $k$ increases by an order of magnitude across the longitudinal domain. 


\begin{tabular}{|c|c|c|c|c|c|}
\hline 3000 & 3500 & 4000 & $\begin{array}{c}4500 \\
\text { depth }(m)\end{array}$ & 5000 & 5500 \\
\hline
\end{tabular}
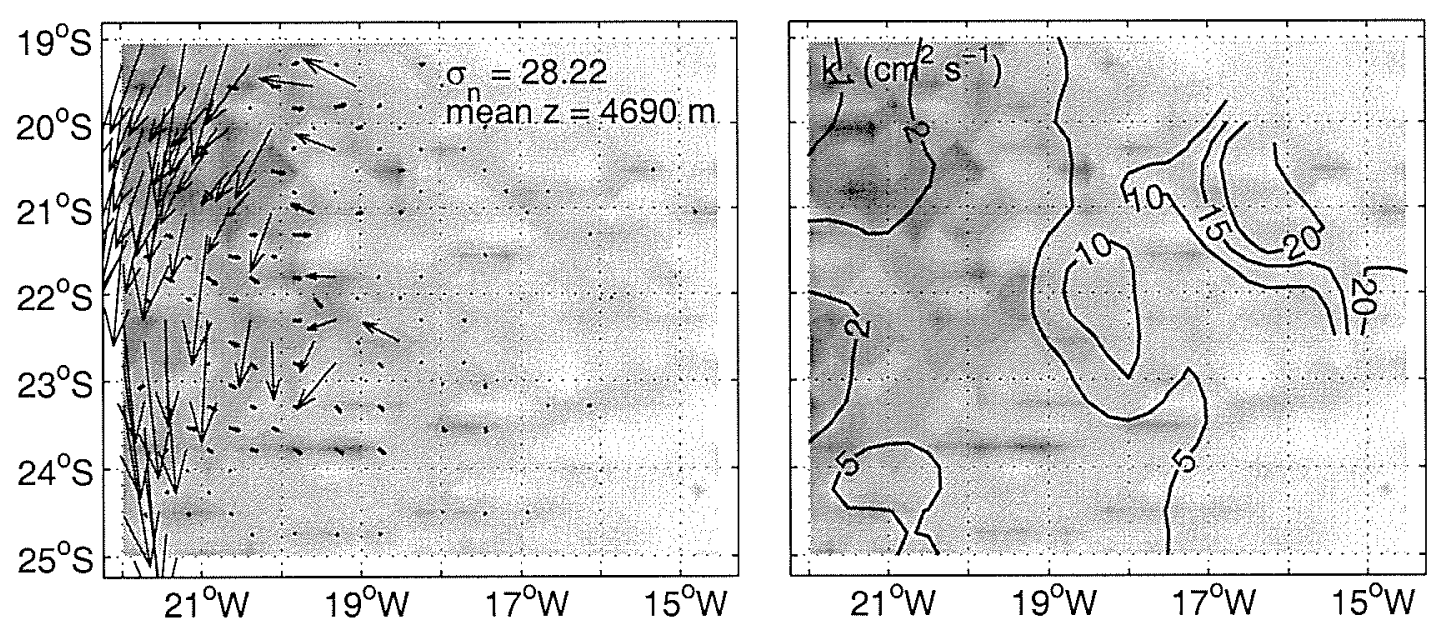

Figure 3.15: Layer-averaged flow and diapycnal diffusivities for the density class $\sigma_{n}>28.21\left(\sigma_{4}>45.99\right)$. The scale of the vectors is enhanced relative to figures 3.13 and 3.14, and the magnitude of the southward flow on the western edge of the domain is (2-4) $\mathrm{mm} \mathrm{s}^{-1}$. Estimates of flow in $\mathrm{FZ}$ canyons are eastward with magnitude $(0.2-0.5) \mathrm{mm} \mathrm{s}^{-1}$. Turbulent diffusivities are shown for the central surface $\sigma_{4}=28.22$, and $k$ increases by a factor of 20 across the longitudinal domain. 


\begin{tabular}{cccccc}
\hline 3000 & 3500 & 4000 & $\begin{array}{c}4500 \\
\text { depth }(\mathrm{m})\end{array}$ & 5000 & 5500 \\
\end{tabular}
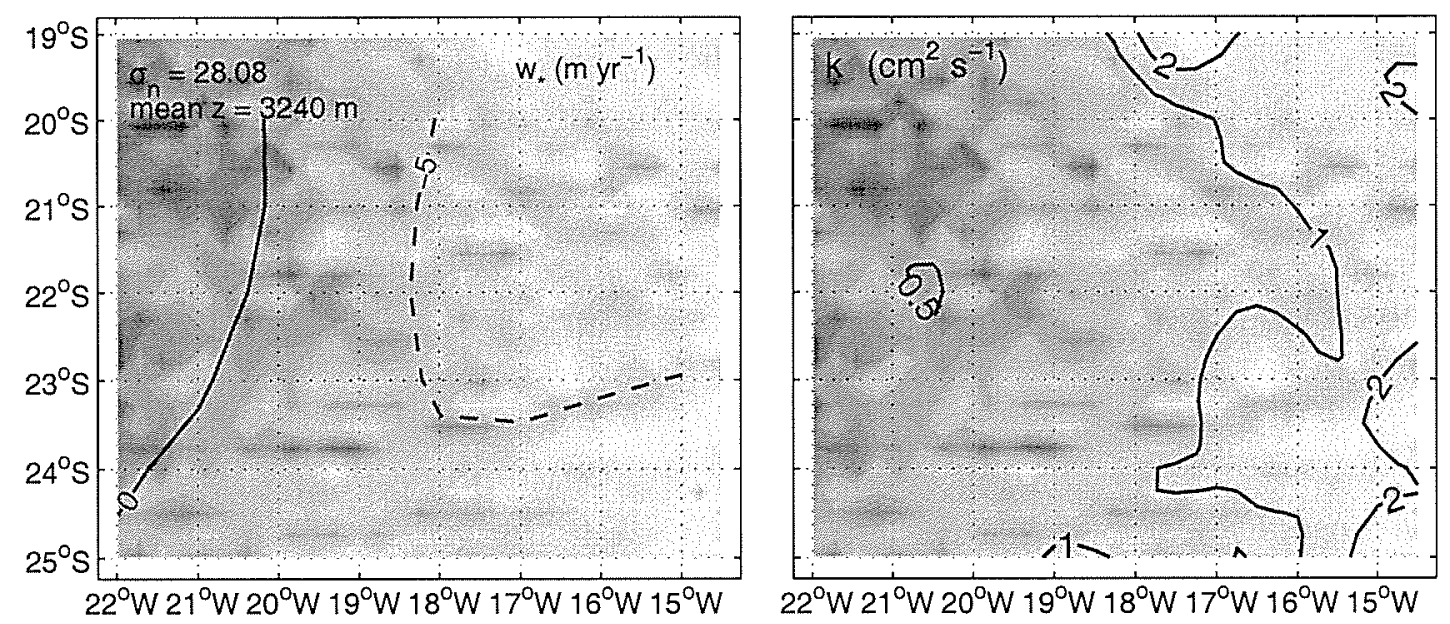

Figure 3.16: Diapycnal advection (left panel) and diapycnal diffusivities (right panel) on the $\sigma_{n}=28.08$ surface $\left(\sigma_{4}=45.83\right.$, mean depth $\left.=3240 \mathrm{~m}\right)$. Downward advection with mean $w_{*}=-(3 \pm 1) \mathrm{m} \mathrm{yr}^{-1}$ and diffusivities of $\mathrm{O}\left(1 \mathrm{~cm}^{2} \mathrm{~s}^{-1}\right)$ characterize the surface. 


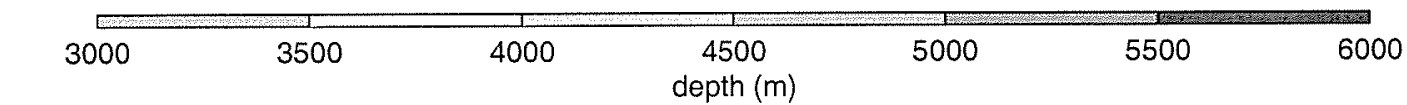
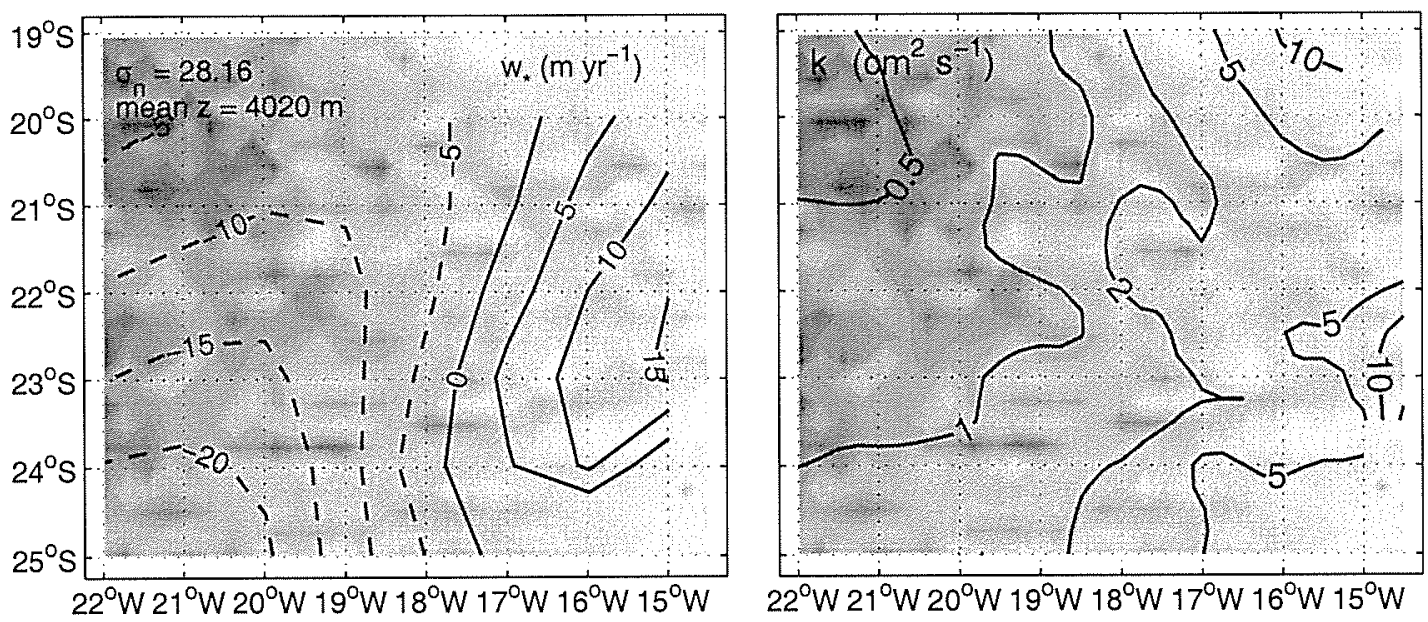

Figure 3.17: Diapycnal advection and diffusivities on the $\sigma_{n}=28.16$ surface $\left(\sigma_{4}=45.94\right.$, mean depth $\left.=4020 \mathrm{~m}\right)$. This surface was the target isopycnal for the tracer release. Downward advection dominates portion of the domain west of $18^{\circ}$ while upwelling is estimated in the eastern region where FZ crests are abundant. Uncertainties of the $w_{*}$ estimates on this surface are roughly $\pm 20 \%$ of the contoured values. Diffusivities above the peaks of FZs are an order of magnitude larger than the values to the west. 


\begin{tabular}{|c|c|c|c|c|c|}
\hline 3000 & 3500 & 4000 & $\begin{array}{c}4500 \\
\text { depth (m) }\end{array}$ & 5000 & 5500 \\
\hline
\end{tabular}
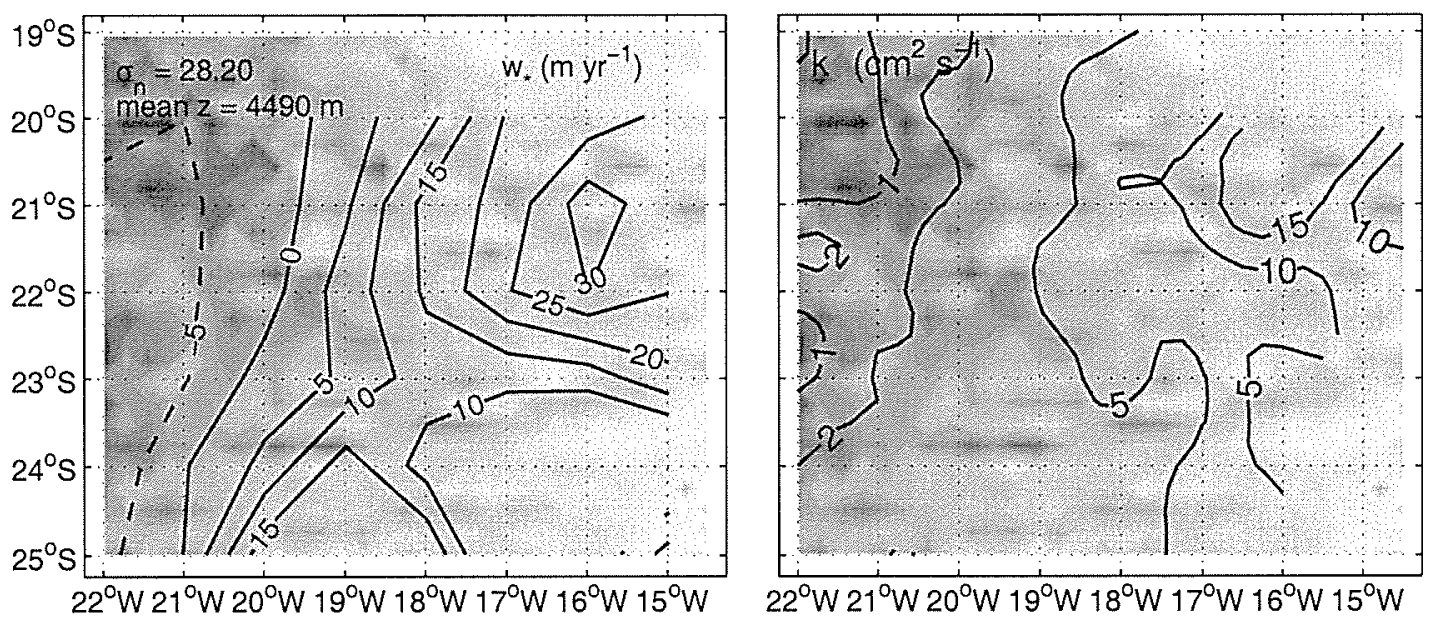

Figure 3.18: Diapycnal advection and diffusivities on the $\sigma_{n}=28.20$ surface $\left(\sigma_{4}=45.98\right.$, mean depth $\left.=4490 \mathrm{~m}\right)$. Upward advection dominates the surface and uncertainties of these $w_{*}$ estimates are roughly $\pm 40 \%$ of the contoured values. 
increase in vertical diffusivity over the longitudinal extent of the inversion domain. For the $\sigma_{n}=28.18$ and $\sigma_{n}=28.22$ surfaces, diffusivities are $\mathrm{O}\left(10 \mathrm{~cm}^{2} \mathrm{~s}^{-1}\right)$ in the stratified water $\left(N^{2} \sim 1 \times 10^{-6} \mathrm{~s}^{-2}\right)$ above the bottom.

The diapycnal advection estimates were made on a series of density surfaces above and below the surfaces of lateral flow. The diapycnal advection estimates through these intermediate surfaces are presented in figures 3.16, 3.17 and 3.18. The $\sigma_{n}=28.08\left(\sigma_{4}=45.83\right)$ surface is characterized by downward advection of mean $w_{*}=-(3 \pm 1) \mathrm{m} \mathrm{yr}^{-1}$, with larger downward pumping occurring toward the east. The deeper surfaces, $\sigma_{n}=28.16\left(\sigma_{4}=45.94\right)$ and $\sigma_{n}=28.20\left(\sigma_{4}=45.98\right)$ are characterized by larger values of advection, $\left|w_{*}\right| \sim 10 \mathrm{~m} \mathrm{yr}^{-1}$, with individual estimates having associated uncertainty of roughly $30 \%$. The $\sigma_{n}=28.16$ surface was the target isopycnal for the tracer release experiment, and downward advection is estimated over the part of the domain occupied by the tracer patch. The 14 month tracer inventory, and a later 28 month inventory, both indicate that the tracer's center of mass sank relative to the target isopycnal (Ledwell et al., 1999). The estimate for the rate of tracer sinking is very uncertain and a detailed comparison is not possible. However, the inversion estimate of sinking in the range -(5 to 20$) \mathrm{m} \mathrm{yr}^{-1}$ is plausible, as assessed with a model of the tracer sinking.

Advection through the eastern range of the tracer surface is upward, as it is for nearly all of the deeper $\sigma_{n}=28.20$ surface. Figures 3.17 and 3.18 show contoured fields of $w_{*}$ that have been highly smoothed. Actual estimates of upward advection in canyons exceed $30 \mathrm{~m} \mathrm{yr}^{-1}$. Where ridge topography occupies each density layer, there is no flow. In this manner, the contoured maps show estimates of $w_{*}$ that have been averaged over both regions of strong upwelling and regions blocked by topography. In FZ canyons, eastward flow is estimated with magnitude $(0.4 \pm 0.2) \mathrm{mm} \mathrm{s}^{-1}$. It is this eastward canyon transport that supplies fluid for the diapycnal export of mass. The matter of canyon flow is discussed further in section 3.4 .

\section{Model Sensitivity}

Various assessments were made of the inverse model's sensitivity to the microstructure derived constraints. One approach taken involves the complete dismissal of the microstructure constraints from the model. In this approach, both the vertical 
diffusivity $k$ and the diapycnal advection $w_{*}$ are treated as formal unknowns. This leaves the hydrographic budgets the full responsibility of estimating the magnitude and direction of the diabatic fluxes, and such estimation relies on the accuracy of the lateral flux divergences. The estimates produced through inversion of this underdetermined model show that estimates of geostrophic flow are rather insensitive to the presence of microstructure constraints, as both variations of the problem give essentially identical lateral flows. However, the diapycnal advection estimates are drastically altered by the dismissal of dissipation data. The unconstrained inversion fails to predict the values of the $w_{*}$ suggested by the constrained inversion. Instead, $\left|w_{*}\right|<0.5 \mathrm{~m} \mathrm{yr}^{-1}$ is predicted everywhere, although a suggestion of western downwelling and eastern upwelling is present. The related estimates of vertical diffusivity also differ drastically from estimates of $k=\left(\Gamma / N^{2}\right) \bar{\epsilon}$. Negative values of diffusivity are numerous, though all estimates are generally indistinguishable from zero given their uncertainty. The application of inequality constraints may have assisted in improving the diffusivity estimates, though such assistance may not have improved estimates of $w_{*}$, as the vertical divergence of the diffusive fluxes (i.e., $w_{*} \sim\left(k N^{2}\right)_{z}$ ) would still have been under constrained.

A substantial improvement was noted when microstructure derived verticaldiffusivities were assigned, despite the absence of a priori $w_{*}$ estimates. This variation of the inversion yielded both lateral and diapycnal flow fields very similar to the constrained estimates. This demonstrates the important role of the thermal advection equations in the inverse problem, as previously noted in the discussion of the data resolution matrix. The microstructure-derived estimate of thermal diffusion $\left(\Gamma\left(\bar{\epsilon} / N^{2}\right) \Theta_{z}\right)$ imposes a strong constraint on the advective terms in the thermal advection budget. When combined with mass conservation, the specification of vertical diffusive-fluxes provides sufficient information to predict the three components of flow. This issue is of consequence in the $w_{*}$ constrained inversion as well, since the influence of the diapycnal advection constraints decreases in deep layers.

\section{Deep Upwelling}

Since all the profiles of modeled dissipation decrease with height above bottom, all a priori estimates specify deep values of $w_{*}<0$. Thus, in regions where up- 


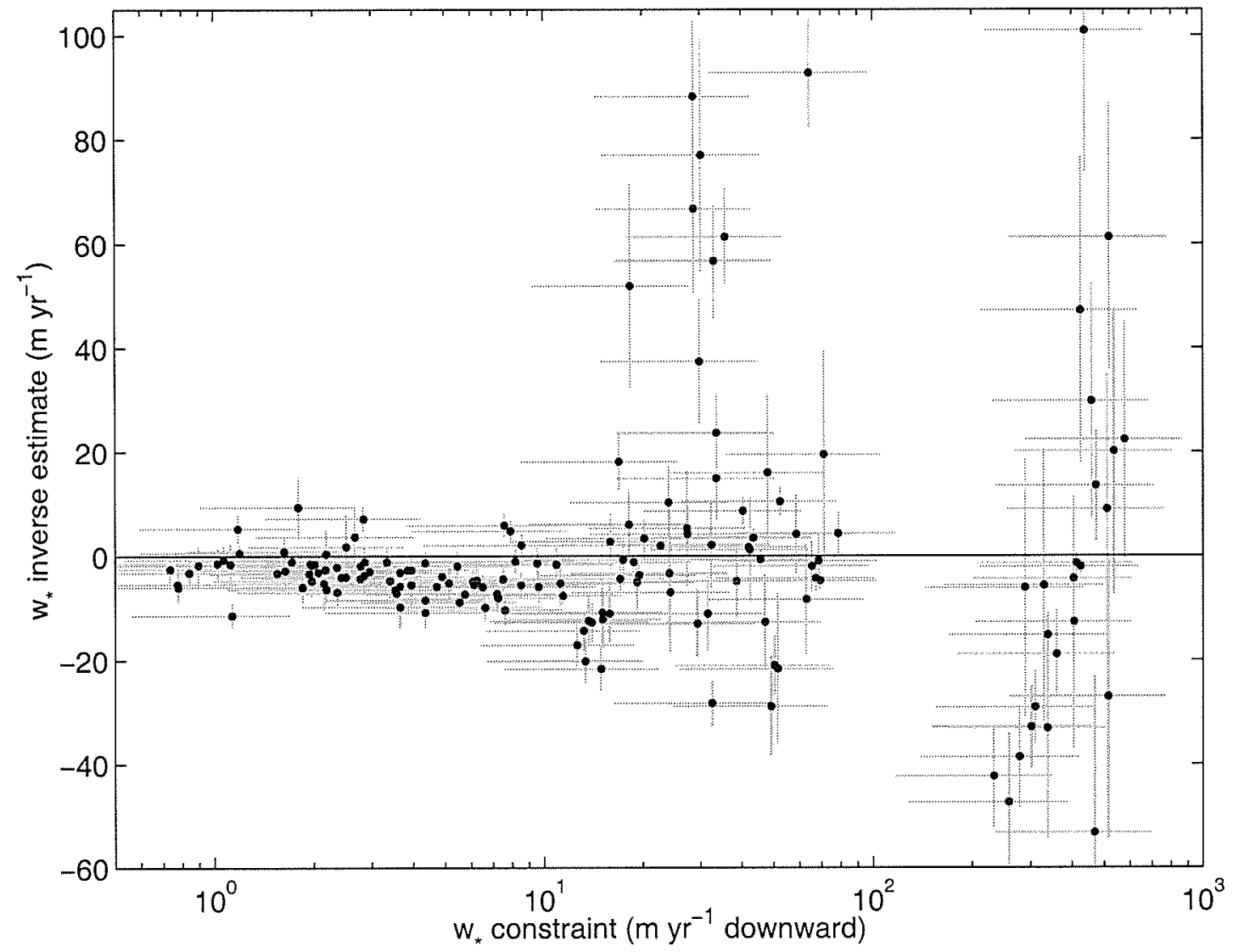

Figure 3.19: Comparison of the a priori estimates of $w_{*}$ with the inverse estimates for the deepest layer of the inverse model $\sigma_{n}>28.21\left(\sigma_{4}>45.99\right)$. All a priori estimates were negative valued, as the modeled profiles of $\bar{\epsilon}$ all decrease with height above bottom. The information provided by mass conservation and thermal advection allow for contradiction of the a priori estimates, producing inverse estimates of upwelling. Standard errors are shown for all estimates. 
welling is found, the inverse estimates of $w_{*}$ relied on the constraints imposed by mass conservation and the balance of thermal advection with vertical diffusion. A presentation of this issue is provided by figure 3.19. Significant contradictions of the a priori $w_{*}$ constraints occur in deep canyons. In these weakly stratified regions, the mixing efficiency parameter $\Gamma$ can not remain near constant. Instead, the quantity $\Gamma=-J_{b} / \epsilon$ must approach zero as $J_{b}=-k_{\rho} N^{2}$ diminishes. In particular, the a priori estimates $w_{*}<-100 \mathrm{~m} \mathrm{yr}^{-1}$ apparent in figure 3.19 are evidence of breakdown in the assumed relation $w_{*} N^{2}=0.2(\partial \bar{\epsilon} / \partial z)$. Thus, there is a tendency for advective constraints to overcome deficiencies in the dissipation model to provide estimates of diapycnal advection in the FZ canyons. Such estimates highlight the strength of the inverse model used in this study. It is only through the combined application of the one-dimensional microstructure constraints and the dominantly two-dimensional hydrographic budgets that a meaningful estimate of the three-dimensional flow is gained.

\subsection{Mixing Rates and Flow in an Abyssal Canyon}

A fundamental feature of the abyssal Brazil Basin is the system of ridges and canyons associated with the FZs of the MAR. Many canyons run zonally for hundreds of kilometers and each canyon is bounded to the north and south by ridges extending 500-1000 m above the canyon floor. The meridional wavelength of the canyon-ridge system is roughly $50 \mathrm{~km}$, and because of this, the flow in these FZs was difficult to resolve in the inverse model calculations. Instead, each canyon is better modeled locally, so that the special geometry of the system can be considered.

The unnamed FZ nearest to the tracer release site was studied closely during the 1997 HRP survey, and a section of hydrography and dissipation was made along the central axis of the canyon. The potential density structure in this canyon is shown in figure 3.20. Isopycnals denser than $\sigma_{n}>28.12\left(\sigma_{4}>45.88\right)$ intersect the canyon floor as it rises toward the MAR. While the isopycnals are relatively flat at the level of the ridge crests, isopycnal slopes increase steeply below the canyon walls. The presence of the steeply sloping isopycnals is coincident with 
a zonal density-gradient, with increasingly lighter water to the east. Although weakly stratified, the vertical density gradient is stable, and $\rho_{z} \sim-7 \times 10^{-6} \mathrm{~kg} \mathrm{~m}^{4}$ greatly exceeds $\rho_{x} \sim-4 \times 10^{-8} \mathrm{~kg} \mathrm{~m}{ }^{4}$. However, any flow along the canyon's axis must be balanced by diapycnal advection through the bounding isopycnal of each water class.

The analysis of $\mathrm{SF}_{6}$ inventories for 1997 and 1998 (Ledwell et al., 1999) suggest that tracer was advected eastward in this canyon and in the neighboring $\mathrm{FZ}$ to the south. Although the precise trajectory of the tracer may not have been entirely along the canyon floor, tracer derived estimates suggest an upcanyon flow of $1 \mathrm{~cm} \mathrm{~s}^{-1}$. Due to a lack of cross-canyon hydrography, this estimate can not be corroborated with estimates of thermal wind shear. However, an integrated temperature-mass budget can provide an estimate of the upcanyon transport between the bottom and a bounding isopycnal.

Consider the integral equations for the advection of potential temperature and mass for a density layer of fluid bounded latitudinally by canyon walls (figure 3.21 ,

$$
\begin{gathered}
\left.\iint d A_{0} \bar{u} \bar{\Theta}\right|_{x_{0}}+\left.\iint d \hat{A} \overline{w_{*}} \bar{\Theta}\right|_{z_{\sigma}}=\iint d \hat{A} F_{\theta}\left(z=z_{\sigma}\right) \\
\left.\iint d A_{0} \bar{u}\right|_{x_{0}}+\left.\iint d \hat{A} \overline{w_{*}}\right|_{z_{\sigma}}=0
\end{gathered}
$$

The starting coordinate of the integral is the western edge of the control volume, taken at the longitude where the height of the bounding isopycnal $z_{\sigma}$ drops below the level of the northern and southern canyon walls, $x=x_{0}$. For the calculation presented here, the $\sigma_{n}=28.20\left(\sigma_{4}=45.98, \theta=0.8^{\circ} \mathrm{C}\right)$ was used. The vertical flux of heat is related to the TKE dissipation rate by $F_{\theta}=-\Gamma \bar{\epsilon} N^{-2} \Theta_{z}$. If we express the volume transport in a simplified form $\left.\iint d A_{0} \bar{u}\right|_{x_{0}}=\bar{u} A_{0}$ and use that the bounding isopycnal is nearly a surface of constant temperature $\hat{\Theta}=0.8^{\circ} \mathrm{C}$, an expression for the flow rate follows,

$$
\left.\bar{u}\right|_{x_{0}}=\frac{-\hat{A}<\Gamma \bar{\epsilon} N^{-2} \Theta_{z}>\left.\right|_{z_{\sigma}}}{A_{0}\left(\Theta_{0}-\hat{\Theta}\right)} .
$$

This expression was evaluated using data from the section of HRP profiles shown in figure 3.20 .

The dissipation model relating the temporally dealiased $\bar{\epsilon}$ to height above bot- 


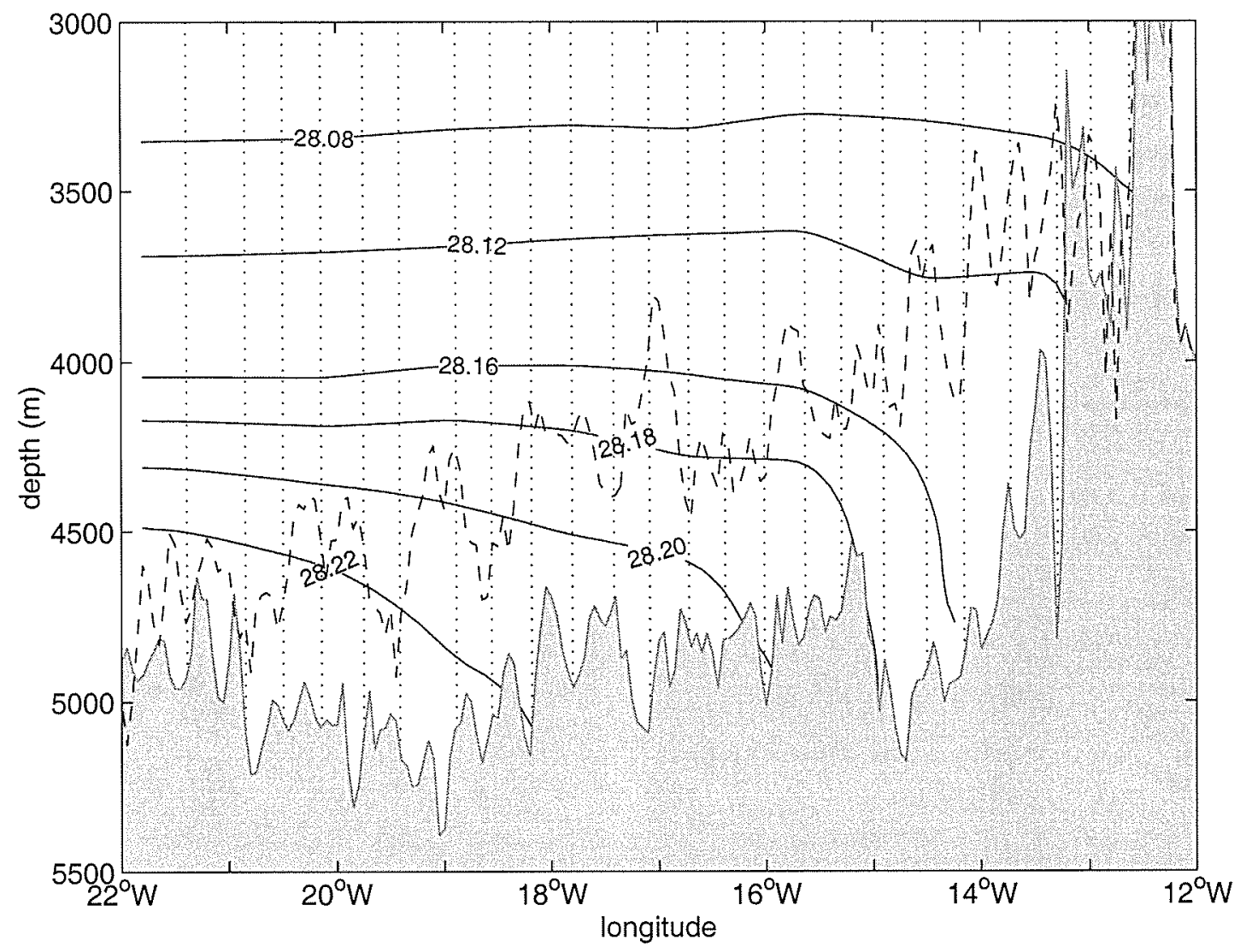

Figure 3.20: Density section in the unnamed canyon near $22^{\circ} \mathrm{S}$. Contours of neutral density are shown, but equivalent values of $\sigma_{4}$ can be found in the text. The observed data were averaged vertically and horizontally to produce the field shown. Shaded bathymetry is the canyon floor, while the bathymetric trace (dashed line) shows the average depth of the canyon walls. Isopycnals denser than $\sigma_{n}>28.12$ intersect to floor of the canyon. Dotted vertical lines denote station profiles. 


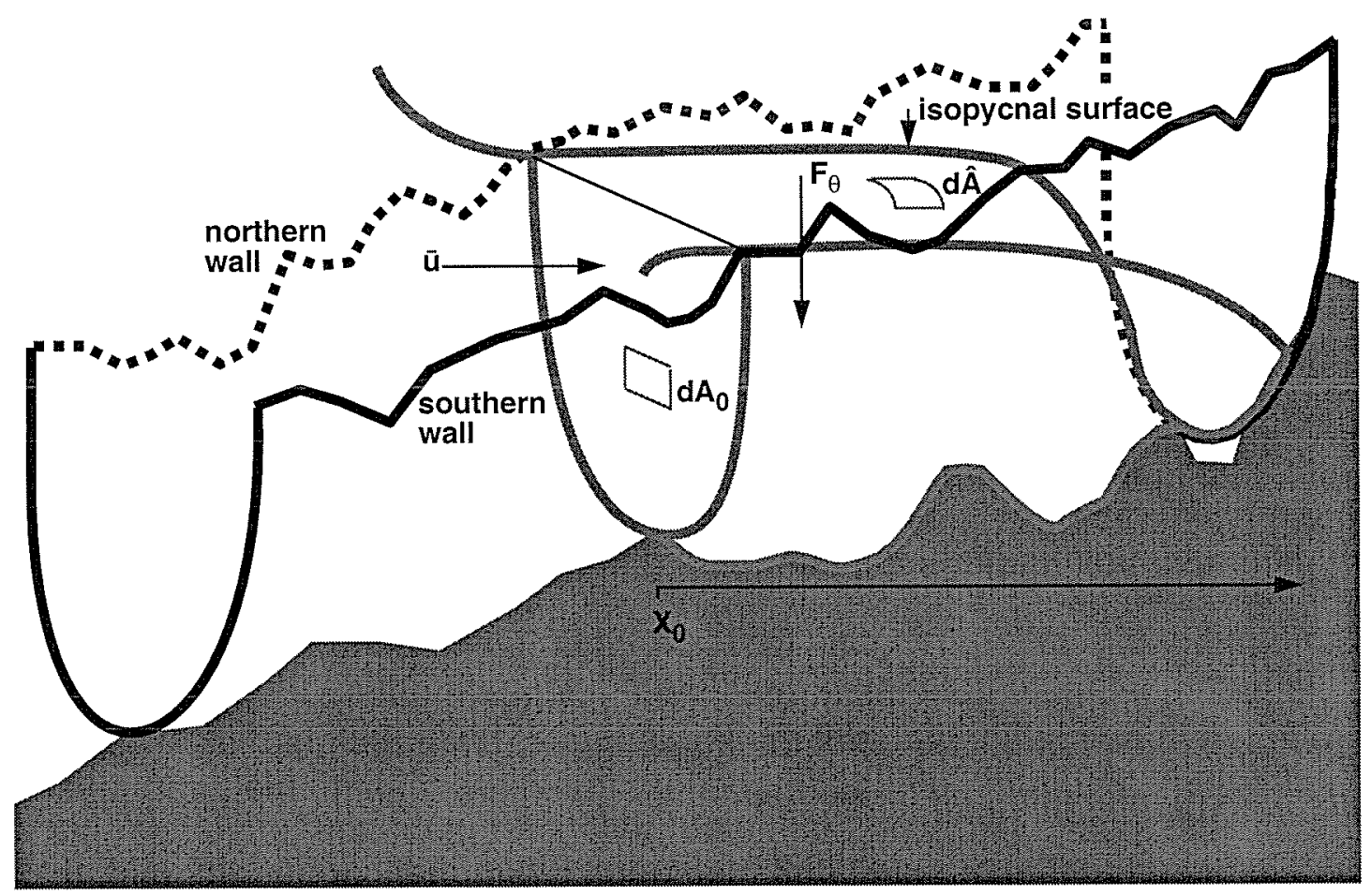

Figure 3.21: Diagram showing the geometry of a control volume contained in the canyon and bounded by a surface of constant density. Differential area along the bounding isopycnal is denoted $d \hat{A}$. Estimates of turbulent diffusivity along the bounding isopycnal imply the flow rate at $x=x_{0}$, through a parabolic plane with differential area $d A_{0}$. 

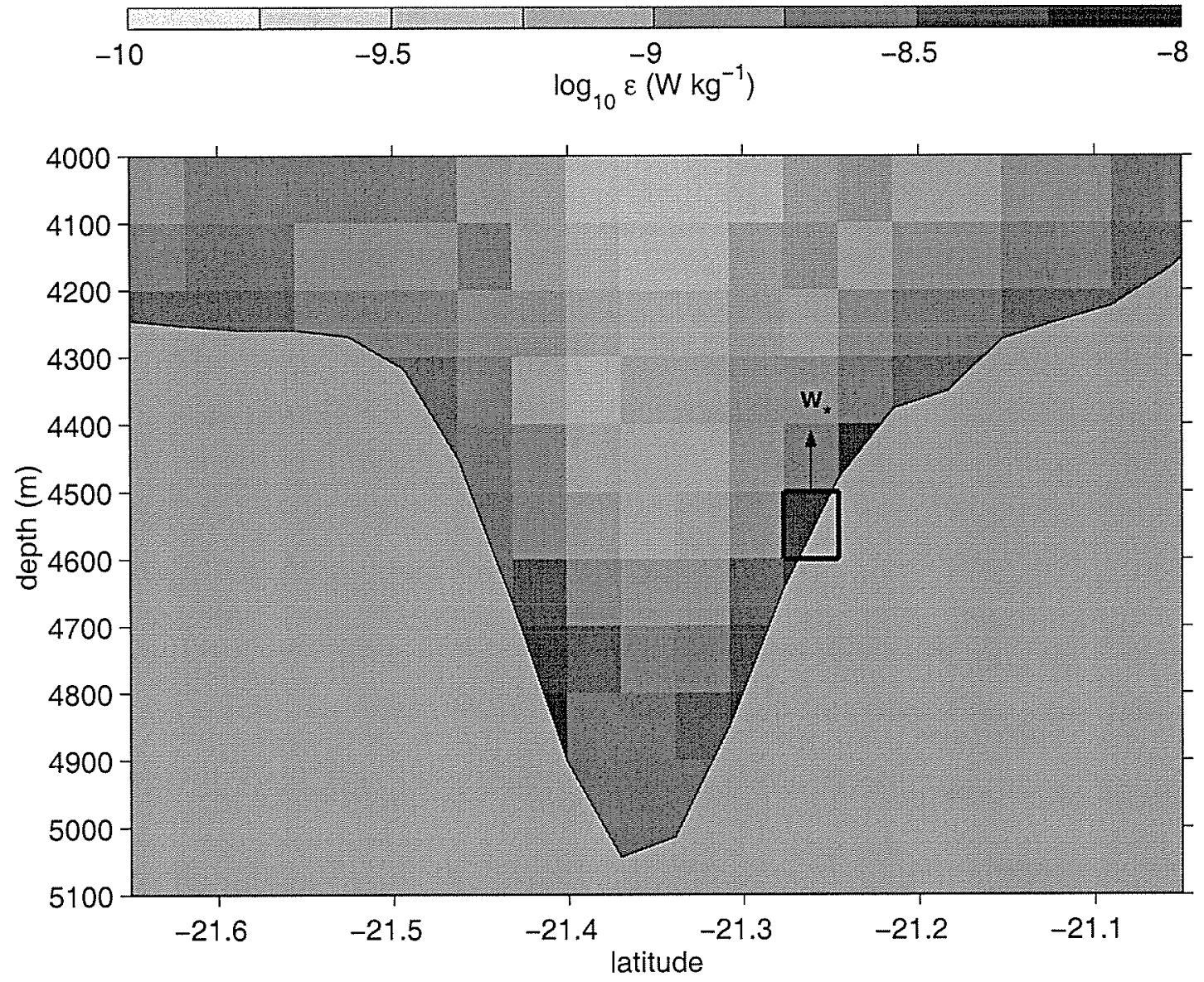

Figure 3.22: Section of dissipation across the canyon at $16^{\circ} \mathrm{W}$. Modeled $\bar{\epsilon}\left(h_{a b}\right)$ profiles were used to generate the dissipation estimates along the floor, slopes, and upper flanks of the canyon. While the vertical derivative of $\bar{\epsilon}\left(h_{a b}\right)$ favors downward advection in the canyon interior, upward $w_{*}$ is favored in proximity to the sloping walls. A control volume taken along the slope will be characterized by a divergent vertical buoyancy-flux, as the turbulent heat-flux will exceed the flux through the seafloor. 


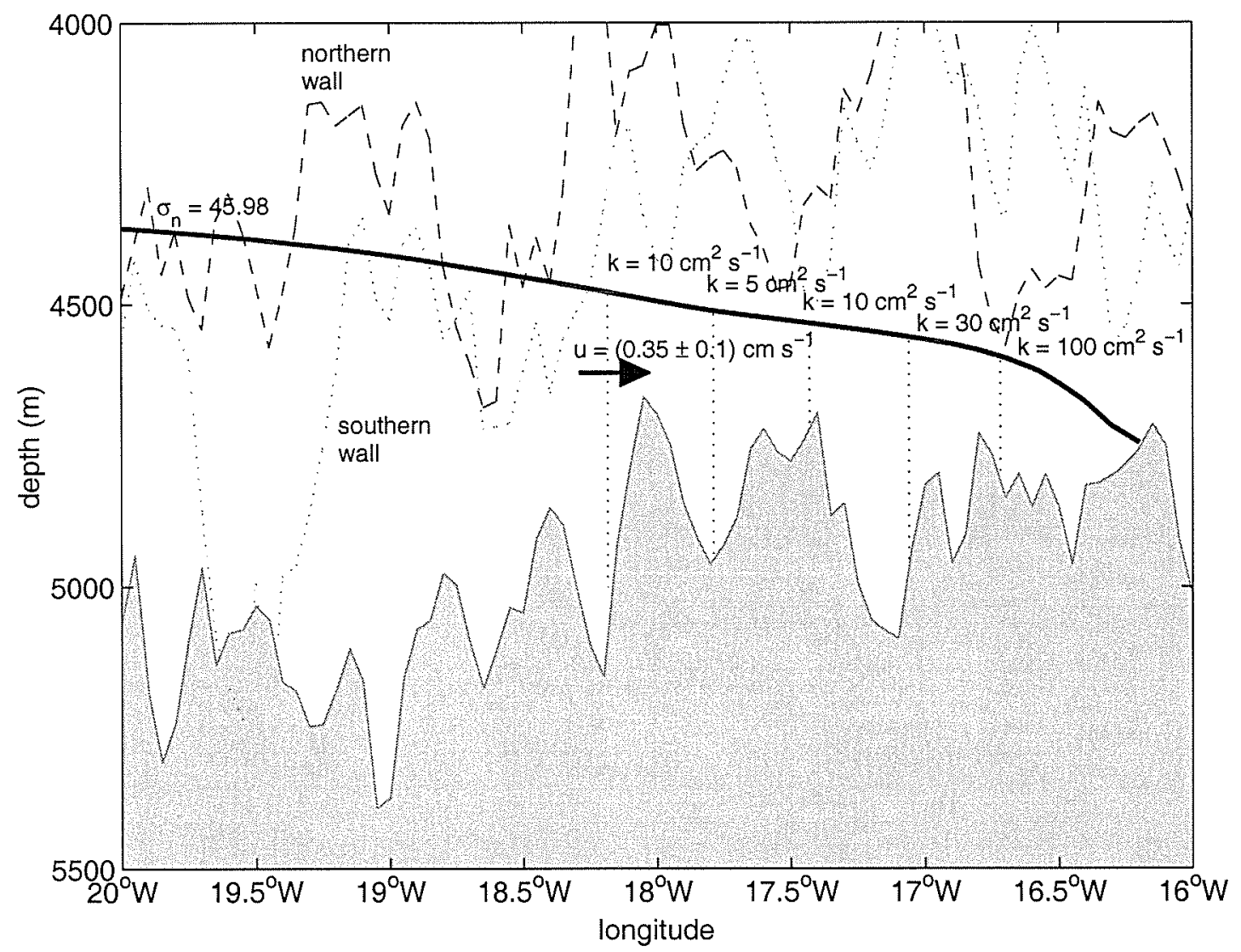

Figure 3.23: Estimates of mixing in the canyon near $22^{\circ} \mathrm{S}$ along the $\sigma_{n}=28.20$ isopycnal. These mixing rates imply an upcanyon flow for the density class $\sigma_{n}>$ $28.20\left(\sigma_{4}=45.98, \theta=0.8^{\circ}\right)$, evaluated at $18^{\circ} \mathrm{W}$ to be $\bar{u}=(0.35 \pm 0.1) \mathrm{cm} \mathrm{s}^{-1}$. The depths of the northern (dashed line) and southern (dotted line) canyon walls are shown above the canyon floor (shaded bathymetry). Vertical dotted lines indicate the section of HRP profiles used for the estimates. 
tom for bathymetric crests, slopes and valleys had been used to estimate turbulent fluxes in the canyon. For each meridional slice across the width of the canyon, $\bar{\epsilon}\left(h_{a b}\right)$ was computed at 2 arc minute increments of latitude. One such meridional slice at $16^{\circ} \mathrm{W}$ is shown in figure 3.22 . This dissipation section shows that the largest values of $\bar{\epsilon}$ occur along the sloping walls of the canyon. At any mid depth location in the canyon, the dissipation rate decreases with height above bottom and $\partial \bar{\epsilon} / \partial z<0$ so that locally diapycnal downwelling is supported. However, any control volume taken in proximity to the sloping canyon walls will favor diapycnal upwelling, as $w_{*} N^{2}=\left(-\partial J_{b} / \partial z\right)>0$ when the no-flux boundary condition is applied at the wall. In this manner, the canyon supports net upwelling, as the buoyancy fluxes at the wall exceed those occurring at locations in the canyon interior.

The diffusivity along the $\sigma_{n}=28.20$ isopycnal was estimated as the ensemble average $k=<\Gamma \epsilon\left(h=h_{\sigma}\right) N^{-2}>$ across the width of the canyon. Error analyses for the $k$ estimates were done using Monte Carlo methods incorporating an uncertainty of $\pm 100 \mathrm{~m}$ in the bottom depth, along with the statistical uncertainty in $\bar{\epsilon}\left(h_{a b}\right)$. Calculated mixing rates are shown schematically in figure 3.23. Height above bottom is clearly related to the magnitude of the diffusivity. Heat fluxes across the $\sigma_{n}=28.20$ surface are roughly $-1 \times 10^{-6} \mathrm{~m}^{\circ} \mathrm{C} \mathrm{s}^{-1}$, with the largest values at the eastern end. While the eastern most edge of the $\sigma_{n}=28.20$ surface experiences the highest mixing rates, the stratification here is very weak $\left(N^{2}=6 \times 10^{-8} \mathrm{~s}^{-2}\right)$, keeping the heat flux in this area comparable to fluxes occurring at greater heights from the bottom.

These mixing rates are used in (3.22) and yield an up-canyon flow rate of $\bar{u}=(3.5 \pm 1) \mathrm{mm} \mathrm{s}^{-1}$ at the western edge of the control volume. This result can be compared with the inverse estimates of $(0.4 \pm 0.2) \mathrm{mm} \mathrm{s}^{-1}$ up-canyon flow. The apparent discrepancy is resolved if volume transports are compared rather than flow rate. The inverse model estimate utilized a subsampled bathymetry grid, such that the dimensions of canyons are only coarsely resolved. The inverse calculations utilized an assumed canyon width of $55 \mathrm{~km}$ over the entire canyon's depth, giving $3 \times 10^{7} \mathrm{~m}^{2}$ as a typical cross section. The section-derived estimate of canyon flow relied on more careful estimates of about $5.5 \times 10^{6} \mathrm{~m}^{2}$ for canyon crosssection. Therefore, the inverse estimates of volume transport are $(0.012 \pm 0.006) \times$ 
$10^{6} \mathrm{~m}^{3} \mathrm{~s}^{-1}$, while the section derived estimate is $(0.019 \pm 0.005) \times 10^{6} \mathrm{~m}^{3} \mathrm{~s}^{-1}$. The two estimates are indistinguishable at the significance level of one standard error.

\subsection{Discussion}

This work has examined the circulation associated with buoyancy forcing occurring in the deep abyss. Specifically, turbulent mixing is enhanced over the rough bathymetry near the MAR and its associated FZs, and this leads to enhanced diapycnal advection in these regions. Furthermore, the vortex stretching achieved by this diapycnal advection serves a significant role by modifying the local balance of planetary vorticity.

The signal of abyssal dissipation was found to have both spatial and temporal content. The spatial distribution of dissipation along an isopycnal is clearly related to height above bottom. In the context of the complex bottom topography associated with FZs, dissipation profiles were found to have differing character for three classes of bathymetry. While dissipation generally increases towards the bottom, levels occurring over the flanks of sloping bathymetry exceed levels occurring on the summits of ridge crests and the floors of canyons. These spatial trends accompany a temporal modulation of dissipation, correlated in phase and amplitude with the barotropic flow of the spring-neap tidal cycle. An attempt was made to model these spatial and temporal trends. The resulting model features a dependence with $h_{a b}$, with the bathymetric classes of ridge crests, canyons, and slopes treated differently. Additionally, the temporal variability of dissipation was modeled according to the energy flux scaling of linear internal waves. This scaling assumes a single dominant bathymetric wavelength to give a relation between $\epsilon$ and the square of the barotropic tidal current. More sophisticated treatments of bottom bathymetry give a slightly larger power dependence, $\epsilon \sim u^{5 / 2}$ (Bell 1975b). The more simple scaling was chosen, based on the lack of any specific knowledge concerning the spectrum of bottom bathymetry on scales less than $O(100 \mathrm{~km})$. Additionally, the temporal dealiasing tends to increase mean dissipation estimates above the observed values of $\epsilon$, as most of the data were collected during neap tide. Thus, the $u^{2}$ scaling is viewed as conservative, as it results in a more conservative enhancement of the neap tide dissipation values than the scalings based 
on assumptions about the bathymetric spectrum.

The modeled dissipation profiles were employed in an inverse model that combines their thermodynamic information with the dynamic constraints implied by the beta spiral and hydrographic budgets. Specifically, the dissipation rate model was used to provide both a measure of the diapycnal diffusivity $k$ and an a priori estimate of the diapycnal advection $w_{*}$. This approach proved highly effective in resolving both the isopycnal and diapycnal components of flow. In particular, the microstructure data were absolutely essential in constraining both the magnitude and direction of the diapycnal flow. In contrast, geostrophic flow was adequately constrained by the application of thermal wind to the hydrographic budgets, with or without the microstructure constraints. The lateral flow divergences in the hydrographic budgets are generally the result of small differences of large flux quantities (Wunsch 1996). Thus, small relative uncertainties in the flux quantities become large errors in the flux divergences. In this manner, the lateral divergences alone provide minimal skill in resolving diabatic terms. It is the combination of the microstructure constraints and the hydrographic budgets that provides skill in estimating the three dimensional flow.

The results of the inverse calculation suggest that while diabatic flow is weak in the much of the interior, stronger levels of turbulence above rough bathymetry lead to enhanced levels of diabatic flow. In layers deeper than $3000 \mathrm{~m}$, a pattern of diapycnal advection exists where downwelling is the preferred mode of vertical advection, as dictated by the slope of the diffusivity profile, $k_{z}<0$. In this sense, the flow over the localized region of the MAR examined in this study opposes the traditional idea of uniform abyssal upwelling suggested by Stommel (1957). However, strong upwelling is found where FZ canyons and ridges shoal to meet the peaks of the MAR. This deep system of downwelling and upwelling defines a zonal circulation that compliments the latitudinal mass transport carried in the deep density-layers. A schematic of the deep circulation through the $28.16<\sigma_{n}<28.20$ density layer is shown in figure 3.24. The downwelling that occurs along the western half of the $\sigma_{n}=28.16$ surface is contrasted by strong upwelling through $\sigma_{n}=28.20$ occurring locally above the eastern limit of the canyons.

To produce a clearer summary of the deep zonal-circulation, a zonal stream function $(\psi)$ was defined according the the latitudinally integrated flow, 


\begin{tabular}{|c|c|c|c|c|c|c|}
\hline ris & का & 15 & म१। & T: & 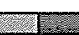 & 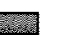 \\
\hline 3000 & 3500 & 4000 & $\begin{array}{c}4500 \\
\text { depth }(m)\end{array}$ & 5000 & 5500 & 6000 \\
\hline
\end{tabular}

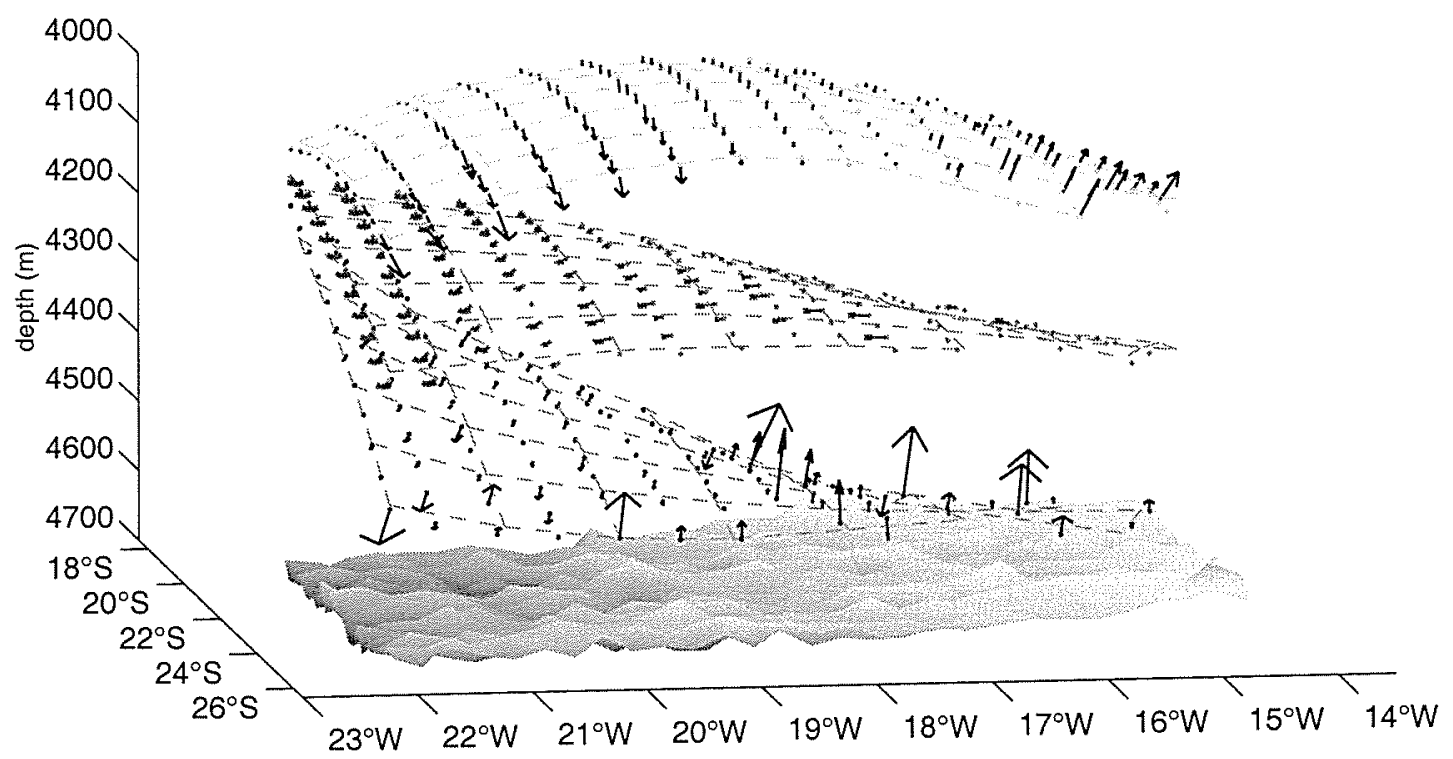

Figure 3.24: A schematic representation of the circulation in the density layer $28.16<\sigma_{n}<28.20$. Diapycnal advection vectors are shown on the upper and lower isopycnals, while lateral flow vectors of $(2-6) \mathrm{mm} \mathrm{s}^{-1}$ are drawn on the middle isopycnal $\sigma_{n}=28.18$. Typical values of downwelling on the upper surface are $w_{*} \sim-15 \mathrm{~m} \mathrm{yr}^{-1}$, while vectors of upwelling across the deep isopycnal are as large as $w_{*}=(100 \pm 50) \mathrm{m} \mathrm{yr}^{-1}$ near the eastern limits of the canyons. The bottom bathymetry is shown as a relief surface, but is squashed to the bottom of the plot to prevent ridges from obscuring the vectors emanating from canyons. 


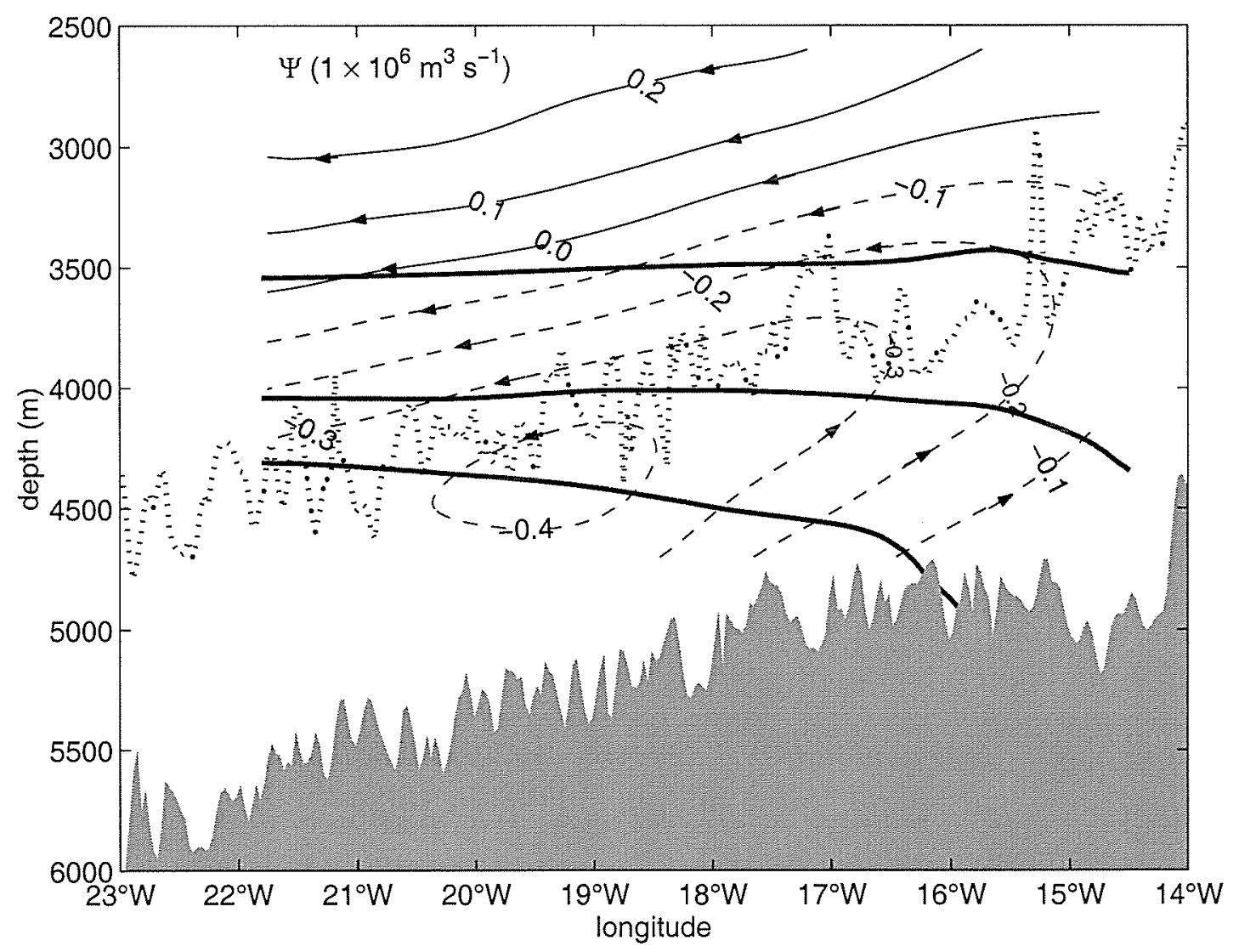

Figure 3.25: The meridionally integrated stream function as estimated through objective analysis of the inverse model solution. Dimensional values of the stream function are contoured, and uncertainties are roughly $\pm 40 \%$. Representative bathymetry of the canyon floors (shaded) and ridge crests (dotted) is shown, as are the $\sigma_{n}=28.10,28.16$ and 28.20 isopycnals. Eastward flow out of the canyons is also upward and is nearly $100 \%$ diapycnal. 


$$
\bar{u} \delta_{y}=-\psi_{z}, \overline{w_{*}} \delta_{y}=\psi_{x}
$$

where the latitudinal limits of integration were taken over the inverse domain $\left(19^{\circ} \mathrm{S}-25^{\circ} \mathrm{S}\right)$

$$
\bar{u}(x, z)=\frac{1}{\int d y} \int d y u(x, y, z), \text { and } \overline{w_{*}}(x, z)=\frac{1}{\int d y} \int d y w_{*}(x, y, z)
$$

The inverse model grid is coarsely spaced in depth, so an objective analysis technique (Bretherton et al., 1976) was used to interpolate the inverse solution and compute the stream function. Prior to the objective analysis, the grid coordinates were nondimensionalized by the assumed correlation scales. For the vertical dimension, the advective-diffusive scale $\hat{z}=k / w_{*} \sim 300 \mathrm{~m}$ was used, while twice the deformation radius was used for a lateral scale, $\hat{x}=2 \cdot R_{d} \sim 30 \mathrm{~km}$. The auto correlation of the stream function was specified to e-fold over three nondimensional correlation radii, $\hat{r}^{2}=(z / \hat{z})^{2}+(x / \hat{x})^{2}$. The resulting smooth stream-function is shown in figure 3.25 relative to representative bathymetry and density field. Below $2500 \mathrm{~m}$ depth, two separate circulations are present. A clockwise circulation shallower than $3500 \mathrm{~m}$ is characterized by western flow and downwelling, while the deeper cell is characterized by canyon upwelling which feeds western flow and downwelling to the west.

The zonal circulation supported by upwelling from abyssal canyons is of primary significance to the tracer release experiment. Though no attempt was made to estimate $\psi$ along the canyon floors, eastern upwelling is clearly supported by eastward canyon flow. The net transport through the a density surface $\left(T_{\sigma}\right)$ can be expressed in terms of the stream function, $T_{\sigma}=\psi\left(x_{w}\right)-\psi\left(x_{e}\right)$, where $\left(x_{e}, x_{w}\right)$ are the eastern and western limits of the surface area integral. The transport calculation is simplified if the eastern limit is taken to be the longitude where the surface intercepts the bottom, so the $\psi\left(x_{e}\right)=0$. For the $\sigma_{n}=28.20\left(\sigma_{4}=45.98\right.$ and $\left.\theta=0.8^{\circ}\right)$ surface, the net diapycnal transport between $\left(19^{\circ} \mathrm{S}, 25^{\circ} \mathrm{S}\right)$ is $(0.34 \pm 0.14) \times 10^{6} \mathrm{~m}^{3} \mathrm{~s}^{-1}$. This is the integrated upwelling over $6^{\circ}$ of latitude, so the actual upwelling occurs in the canyons present in the domain. Assuming an upcanyon flow of $0.35 \mathrm{~cm} \mathrm{~s}^{-1}$ is typical of canyons that are 
$500 \mathrm{~m}$ deep and $30 \mathrm{~km}$ wide, then 6-7 canyons would carry the required mass for upwelling, which is quite consistent with the number of canyons in the region. The transport of the zonal cell compares to a meridional (southern) transport of $-(0.34 \pm 0.05) \times 10^{6} \mathrm{~m}^{3} \mathrm{~s}^{-1}$ for the density layers deeper than $3200 \mathrm{~m}\left(\sigma_{n}>28.08\right)$. Thus, the zonal cell is complimentary to the meridional circulation.

The basin scale mass-budget for water of density class $\sigma_{n}>45.98\left(\theta<0.8^{\circ}\right)$ was considered by Hogg et al. (1982). This budget suggests that in the latitude range between $30^{\circ} \mathrm{S}$ and the equator, $(3-3.5) \times 10^{6} \mathrm{~m}^{3} \mathrm{~s}^{-1}$ of net upwelling occurs across the $\sigma_{n}=45.98$ surface. If the physics represented by the stream function shown in figure 3.25 are applicable to the basin scale, then the data described here would suggest an upwelling across $\sigma_{n}=45.98$ of $(1.7 \pm 0.7) \times 10^{6} \mathrm{~m}^{3} \mathrm{~s}^{-1}$. This number is in the general range needed to close the Hogg et al. mass budget. Maps of sea floor bathymetry show a network of 20-30 FZs occupying the entire latitudinal extent of the Brazil Basin. Additionally, the character of barotropic tidal energy does not significantly vary along the axis of the MAR. Treating these as proxies for turbulent dissipation, the spatial distribution of mixing depicted in figure 3.2, and the associated circulation cell, may be characteristic of the greater basin. Thus, the inferred closure of the large-scale mass budget is plausible.

The work described here demonstrates that direct measures of mixing (both tracer and microstructure derived) can be combined with hydrographic observations to give an estimate for circulation that is consistent with other large-scale budgets. This is in contrast to many earlier attempts to reconcile local dissipation estimates with mixing rates inferred from advective-diffusive budgets. A notable difference between the current approach and early treatments of dissipation data rests with the combined application of both microstructure and conventional inverse-techniques. While direct observations of mixing tend to give detailed information on local vertical exchange processes, large-scale hydrographic budgets predict only the mean of diabatic forcing over large scales. Neither approach alone would give a meaningful measure of the full three-dimensional circulation.

In summary, the solution of abyssal flow presented here demonstrates that the abyssal circulation problem is far from being solved. Buoyancy forcing provided by turbulent mixing may be entirely responsible for the mean abyssal circulation, and the observations described constitute the only available observations of 
deep dissipation. If the global abyssal-circulation is to be known, the problem of determining the distribution of deep mixing should take precedence. 


\section{Chapter 4}

\section{Conclusions}

The general issue of vertical mixing in the ocean was considered using estimates of dissipation rates derived from observations of microstructure. These dissipation rates serve an important role in the thermodynamic equations for heat and energy. They provide estimates of buoyancy forcing that would otherwise not be available. The eddy diffusivities of heat and density are directly related to the dissipation rates, and the divergence of these mixing rates with depth dictate the magnitude and direction of the diapycnal advection. The diapycnal advection specifies the component of flow perpendicular to surfaces of constant density. It is the physical mechanism that allows buoyancy forces to stretch fluid columns and modify the potential vorticity of the lateral circulation.

One specific problem considered involved the role of double-diffusive mixing in the North Atlantic thermocline. The dissipation associated with salt-finger convection was assessed relative to background levels of turbulent dissipation associated with internal-wave shear. To diagnose the strength of salt-finger mixing, a model related to the ratio of the diffusivities for heat and buoyancy ( $\Gamma$, "the dissipation ratio") was considered. By examining the dissipation ratio in a parameter space of density ratio $\left(R_{\rho}\right)$ and Richardson number $(R i)$, we are able to discern the signal of salt fingers even under conditions where turbulent mixing is also occurring. While the model for turbulence describes most dissipation occurring when $R i<1$, dissipation at larger $R i$ is better described by the saltfinger model. Based on the results of the parameter space analysis, a method for estimating the salt-finger enhancement of the diapycnal haline diffusivity $\left(k_{s}\right)$ 
over the thermal diffusivity $\left(k_{\theta}\right)$ was proposed. During April 1992 at the NATRE site, we find $k_{\theta}=(0.08 \pm 0.01) \mathrm{cm}^{2} \mathrm{~s}^{-1}$ and $k_{s}=(0.13 \pm 0.01) \mathrm{cm}^{2} \mathrm{~s}^{-1}$ for the neutral density surface local to the tracer release isopycnal $\left(\sigma_{\theta} \sim 26.75 \mathrm{~kg} \mathrm{~m}^{3}\right.$, $z \sim 300 \mathrm{~m}$ ). The flux divergence of buoyancy was also computed, giving a diapycnal advection estimate of $w_{*}=-(1.7 \pm 1.2) \mathrm{m} \mathrm{yr}^{-1}$. Moreover, divergence of vertical buoyancy flux was dominated by the haline component. For comparison, a tracer method inferred a diffusivity of $k_{s}=(0.12 \pm 0.02) \mathrm{cm}^{2} \mathrm{~s}^{-1}$ and a diapycnal velocity of $w_{*}=-(3 \pm 1) \mathrm{m} \mathrm{yr}^{-1}$ at this site. The above numbers were contrasted to diffusivity estimates derived from turbulence theory alone. Best agreement between tracer-inferred mixing rates and microstructure based estimates was achieved when the salt-finger enhancement of $k_{s}$ was taken into account.

A second problem considered the turbulent mixing occurring above rough bathymetry in the abyssal Brazil Basin. Levels of enhanced dissipation $(\epsilon)$ are clearly related to height above bottom $\left(h_{a b}\right)$, and both spatial and temporal trends are present. The mixing levels along sloping bathymetry exceed the levels observed on ridge crests and canyon floors. Additionally, mixing levels modulate in phase with the spring-neap cycle of tides. Internal waves generation by barotropic tidal-flow over topography are the only mechanism capable of suppling the energy needed to support the observed dissipation rates; frictional boundary layer processes are not significant. A model of the dissipation rate $\epsilon\left(h_{a b}\right)$ was derived from data that was temporally dealiased using an internal-wave energy scaling with a record of the barotropic tides, and the model retains a spatial dependence associated with the distribution of bathymetry. The modeled dissipation rates were used to specify the turbulent diffusivity $(k)$ and constrain the diapycnal advection $\left(w_{*}\right)$ in an inverse model for the steady circulation. This inverse model uses both beta-spiral and integrated forms of the advective budgets for heat, mass and vorticity, and provides sufficient information to resolve the full three-dimensional flow. The inverse model solution reveals the presence of a deep circulation with zonal character. On isopycnals above the level of fracture zone crests, flow is westward and fluid is downwelled at rates of $w_{*}=-(10-20) \mathrm{m} \mathrm{yr}^{-1}$. Along deeper isopycnals, fluid is carried eastward in canyons and then upwelled at rates exceeding $w_{*}=30 \mathrm{~m} \mathrm{yr}^{-1}$ where the abyssal bottom shoals to meet the Mid Atlantic 
Ridge. This circulation accounts for $(0.3 \pm 0.1) \times 10^{6} \mathrm{~m}^{3} \mathrm{~s}^{-1}$ of local upwelling for the water class with potential temperature $\theta<0.8^{\circ} \mathrm{C}$. This local result scales properly to account for the basin scale budget of Antarctic Bottom Water.

\subsection{Salt-Finger Mixing}

Much of the work presented on salt-finger mixing dealt with the problem of detection. The dissipation signal of fingers proved to be subtle. Both the thermal and kinetic energy dissipation estimates were needed, and the bimodality of the high $\chi$ statistics of $\epsilon$ provided guidance. With the parameter space provided by $R_{\rho}, R i$ and the dissipation ratio $\Gamma=\left(\chi N^{2}\right)\left(2 \epsilon \Theta_{z}^{2}\right)^{-1}$, the signal of salt fingers was clearly discernable from the signal of turbulence. The dissipation-ratio method provides an excellent discrimination technique.

Previous observations of salt-finger mixing were under appreciated due to the lack of a discrimination method. The most notable observations of salt-finger mixing were made in a $10^{6} \mathrm{~km}^{2}$ region of the tropical Atlantic just east of Barbados. This region of ocean experiences such intense mixing by salt fingers that a permanent thermohaline staircase is maintained. Observations of vertical microstructure (Gregg and Sanford, 1987; Gregg 1988) and towed microstructure (Lueck 1987; Marmorino et al., 1987) were made during a 1985 field program (CSALT). Both classes of data revealed active mixing in a series of convecting layers, separated by sharp interfaces. In terms of the processes governing shear and convective stability, the vertical microstructure measurements are crucial, as the towed measurements cannot resolve the stability parameters $R i$ and $R_{\rho}$. The dissipation rates estimated from the vertical microstructure exhibit the low- $\epsilon$ /high- $\chi$ nature of the salt-finger signal observed at the NATRE site. Furthermore, the ensemble statistics reported by Gregg (1988) indicate values of the dissipation ratio $\Gamma>1$, implicating that nearly all mixing at the CSALT site occurs with a buoyancy-flux ratio typical of fingers, $\left(\alpha F_{b} / \beta F_{s}\right)<1$. Gregg also reports a vertically averaged thermal diffusivity of $k_{\theta}=\chi /\left(2 \Theta_{z}^{2}\right) \sim 0.4 \mathrm{~cm}^{2} \mathrm{~s}^{-1}$ for the depth range between $300 \mathrm{~m}$ and $600 \mathrm{~m}$. If salt fingers dominate the vertical mixing, then for an average density ratio of 1.7 , the enhanced haline diffusivity is $k_{s} \geq 1 \mathrm{~cm}^{2} \mathrm{~s}^{-1}$. A haline mixing rate of this magnitude implies that the diffusive fluxes of salt fingers play 
a major role in the region's advective budget of fresh water.

The issue of water mass transformation is central to studies of ocean variability, and salt-fingers reserve a special role in this matter. Since fingers differentially flux temperature and salinity, they provide a mechanism of water mass conversion that differs from mixing by turbulent and isopycnal processes. One measure of water mass conversion is the slope of the temperature-salinity relationship $(\alpha / \beta)(\partial \Theta / \partial S)$, as scaled by the expansion coefficients. Distinct trends in $\Theta-S$ slope are exhibited by different modification processes. Vertical mixing by turbulence acts to equilibrate $(\alpha / \beta) \Theta_{S}$ with the vertical density-ratio, while isopycnal stirring (i.e., by baroclinic eddies) acts to achieve a $\Theta-S$ slope of unity. Salt fingers, with their associated differential heat and salt fluxes, act to modify the $\Theta-S$ slope in the direction of the finger buoyancy-flux ratio, say $(\alpha / \beta) \Theta_{S}=0.6$. An equation for $\Theta-S$ slope can be written as a frequency weighted sum of the three conversion values, subject to the condition that the frequency weights add to unity. Simple inspection of $\Theta-S$ does not serve to dictate the relative importance of these processes, as two equations for the three unknown frequencies is underdetermined. The salt finger mechanism is significant because it is the most efficient means of decreasing the $\Theta-S$ slope. For this reason, Schmitt (1999) identifies salt fingers as a strong consumer of "spice" $(\Theta-S$ variance on a density surface). Specifically, salt finger mixing acts to dilute $\Theta-S$ anomalies.

A number of studies have examined the problem of water mass conversion as fluid is subducted into the thermocline from the mixed layer. The subject was initiated by Iselin (1939), who pointed out the correlation between the horizontal $\Theta-S$ structure of the winter mixed layer and the vertical $\Theta-S$ structure of the thermocline. This work was extended by Stommel (1993), who argued that all conversion occurs in the mixed layer, and that this fluid is adiabatically subducted into the gyre. A contrary view is that fluid with arbitrary $\Theta-S$ relation will be subducted, but a combination of conversion processes will adjust the $\Theta-S$ slope to a value near 2, as observed throughout the subtropical gyres (Schmitt 1981; Stommel 1993). In particular, Schmitt (1981) demonstrates the effectiveness of salt fingers in restoring $\Theta-S$ anomalies to values of $(\alpha / \beta) \Theta_{S}=2$. Recent mixed-layer observations by Rudnick and Ferrari (1999) show that a buoyancy compensated $\Theta-S$ relation with horizontal density-ratio $(\alpha / \beta)(\nabla \Theta / \nabla S)=1$ 
can exist with self-similar scales between $1000 \mathrm{~km}$ and $10 \mathrm{~m}$; a feature worthy of the description "truly amazing". Their observations strengthen the claim that interior mixing processes are needed to maintain a thermocline $\Theta-S$ slope of 2 .

A possible avenue for investigating the role of salt fingers in water-mass conversion involves analysis of the density-ratio evolution equation, formulated by Schmitt (1990). Schmitt's equation dictates the Lagrangian rate of change of $R_{\rho}$ relative to flow along an isopycnal. This balance indicates that $R_{\rho}$ can be modified by shear-advection of isopycnal salinity-gradients (an adiabatic process), or by mixing across (turbulence or fingers) or along (eddy stirring) isopycnals. Specifically, the governing equation is

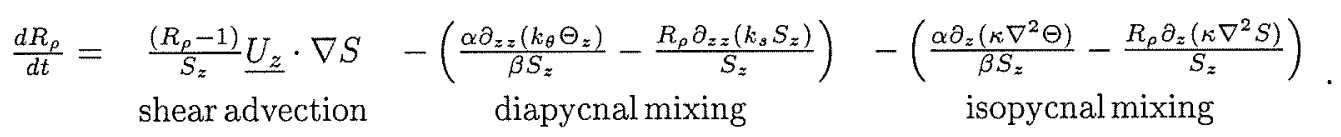

The relative size of these terms were assessed for the tracer level at the NATRE site. The rough estimate for the shear advection term was $0.01 \mathrm{yr}^{-1}$, while the diapycnal mixing term was $-0.04 \mathrm{yr}^{-1}$ when the salt-finger enhancement of $k_{s}$ is accounted for. The lateral mixing term was estimated as $0.002 \mathrm{yr}^{-1}$ when a vertical change in the lateral diffusivity was taken as $(\Delta \kappa / \Delta z) \sim\left(100 \mathrm{~m}^{2} \mathrm{~s}^{-1} / 500 \mathrm{~m}\right)$. Vertical mixing by salt fingers is the most significant modification mechanism at the tracer depth of the NATRE site. However, advection length-scales of $O(1 \times$ $\left.10^{6} \mathrm{~m}\right)$ and time-scales of $\mathrm{O}(10 \mathrm{yr})$ characterize the subduction journey of fluid parcels to mid-thermocline depths. Thus, any modification rates calculated for the NATRE site are too localized to be generalized to gyre scales. Additional observational work spanning the scale of the North Atlantic gyre is needed. Float programs currently being implemented may partially fulfill this need.

\subsection{Abyssal Dissipation}

The calculations presented for mixing and flow near rough bathymetry in the Brazil Basin dictate that the distribution of abyssal buoyancy-forcing is far from uniform. While the study strictly considered data from a small geographic region, the bathymetric features present are ubiquitous over the basin of the global ocean, as are the tides. If, as suggested by Munk and Wunsch (1998), the tides 
are the dominant source of mechanical energy for abyssal mixing, the pattern of dissipation discussed for this work is likely to be representative rather than exceptional.

The suggestion that buoyancy forcing is not uniformly distributed in the abyss is supported by float observations from the Brazil Basin. Hogg and Owens (1999) report that float trajectories over the period 1992 to 1996 are dominantly zonal over much of the basin. Early experiments by Stommel, Arons and Faller (SAF, 1958) demonstrated the tendency for zonal flow when the deep interior is characterized by a strong zonal gradient in the magnitude of buoyancy forcing. Specifically, the SAF experiments showed that zonal jets would transport mass between the deep western boundary current and isolated sources of upwelling to the east, while meridional motions were inhibited in the interior due to the absence of buoyancy forces. The zonal circulation system of the abyssal canyons is similar to the zonal flows described observed by SAF. A realistic model of abyssal flow may more closely resemble these zonal circulations, rather than the common picture of meridional interior flow forced by uniformly distributed upwelling.

A natural extension of the Brazil Basin results involves parameterizing abyssal dissipation in circulation models, according to the spatial distributions of seafloor topography and barotropic tidal energy. A number of fruitful directions could be pursued, and despite the "fiction" of such parameterized mixing, it would surely be an improvement over models with arbitrary distributions of buoyancy forcing. A foremost goal would be mapping the global distribution of deep dissipation. This would provide a proxy for buoyancy forcing via the thermodynamic relation between buoyancy flux and $\epsilon$. Such a diagnosis would be complete only in the context of a mass conserving flow, as the dissipation model alone does not directly predict the distribution of upwelling. The implementation of such a parameterization should not be difficult, good models of the barotropic tides are available (Egbert et al., 1994), as are high resolution maps of sea floor bathymetry (Smith and Sandwell, 1997).

A second issue of direct relevance to the problem of long-term climate involves paleo-mixing. As discussed by Munk and Wunsch (1998), both the distribution of sea floor topography and the lunar forcing are known to have changed radically over geologic time. Paleo representations of the Earth-Moon system, combined 
with information on past arrangements of tectonic plates, would provide the minimal ingredients for drawing maps of paleo-dissipation. The problem of buoyancy forcing is more complicated, as dissipation estimates must be combined with a measure of stratification. This later step may also be pursued, given some knowledge of the deep temperature structure inferred from benthic foraminifera.

Far reaching applications of the general problem considered here go beyond the circulation of the Earth's ocean, to the hypothesized ocean on Europa, the ice covered moon of Jupiter. The tidal forcing of such an ocean would be impressive by Earthly standards, as the tidal energy of Galilean system maintains, through frictional dissipation on Io, the most geologically active body in the solar system. The existence of stratification and bathymetry, whether at the upper ice-boundary or at the planetary interior, would provide the necessary ingredients for wave generation. 


\section{References}

Baker, M. A., and C. H. Gibson, 1987: Sampling turbulence in the stratified ocean: Statistical consequences of strong intermittency. J. Phys. Oceanogr., $17,1817-1836$.

Barton, E. D., and A. E. Hill, 1989: Abyssal flow through the Amirante Trench (Western Indian Ocean). Deep-Sea Res., 36, 1121-1126.

Bell, T. H., 1975a: Lee waves in stratified flows with simple harmonic time dependence. J. Fluid Mech., 67, 705-722.

Bell, T. H., 1975b: Topographically generated internal waves in the open ocean. J. Geophys. Res., 80, 320-327.

Bevington, P. R, and D. K. Robinson, 1992: Data Reduction and Error Analysis for the Physical Sciences, McGraw-Hill, 328 pp.

Bretherton, F. P., R. E. Davis and C. B. Fandry, 1976: A technique for objective analysis and design of oceanographic experiments applied to MODE-73. Deep-Sea Res., 23, 559-582.

Bryan, F., 1987: Parameter sensitivity of primitive equation ocean general circulation models. J. Phys. Oceanogr. 17, 970-985.

Cox, M. D., 1989: An idealized model of the world ocean. Part 1: The global scale water masses. J. Phys. Oceanogr., 19, 1730-1752.

Davis, R. E., 1994: The Osborn-Cox Model. J. Phys. Oceanogr., 24, 2560-2576.

Davis, R. E., 1996: Sampling turbulent dissipation. J. Phys. Oceanogr., 246, 341-358. 
Dillon, T. M., 1984: Vertical Overturns: A comparison of Thorpe and Ozmidov Length Scales. J. Geophys. Res., 87, 9601-9613.

Efron, B., and G. Gong, 1983: A leisurely look at the Bootstrap, the Jackknife, and Cross-Validation. The American Stat., 37, 36-48.

Egbert, G. D., A. F. Bennett, and M. G. G. Foreman, 1994: TOPEX/ POSEIDON tides estimated using a global inverse model. J. Geophys. Res. 99, 24821-24852.

Fukumori, I., 1991: Circulation about the Mediterranean Tongue: An analysis of an EOF-based model ocean. Prog. Oceanog., 27, 197-224.

Gargett, A. E., and B. Ferron, 1996: The effects of differential vertical diffusion of $\mathrm{T}$ and $\mathrm{S}$ in a box model of thermohaline circulation. J. Marine Res., 54, 827-866.

Garrett, C., 1991: Marginal mixing theories. Atmos.-Ocean, 29, 313-339.

Garrett, C. J. R., and W. H. Munk, 1975: Space-time scales of internal waves: A progress report. J. Geophys. Res., 80, 291-297.

Gibson, C. H., 1982: Alternative interpretations for microstructure patches in the thermocline. J. Phys, Oceanogr., 12, 374-383.

Gill, A. E., 1982: Atmosphere-Ocean Dynamics. Academic Press. 662pp.

Gnanadesikan, A., 1999: A simple predictive model for the structure of the oceanic pycnocline. Science, 283, 2077-2079.

Gregg, M. C., 1980: Microstructure patches in the thermocline. J. Phys. Oceanogr., $10,915-943$.

Gregg, M. C., 1987: Diapycnal mixing in the thermocline: A review. J. Geophys. Res., 92, 5249-5286.

Gregg, M. C., 1988: Mixing in the thermohaline staircase east of Barbados. In: Small-Scale Turbulence and Mixing in the Ocean, J. Nihoul and B. Jamart, editors, Elsevier Oceanography Series, 46, Elsevier, New York, pp. 453-470. 
Gregg, M. C., and T. Sanford, 1980: Signatures of mixing from the Bermuda Slope, the Sargasso Sea, and the Gulf Stream. J. Phys. Oceanogr., 10, 105-127.

Gregg, M. C., H. Peters, J. C. Wesson, N. S. Oakey, and T. J. Shay, 1985: Intensive measurements of turbulence and shear in the equatorial undercurrent. Nature, 318, 140-144.

Gregg, M. C., and T. Sanford, 1987: Shear and turbulence in a thermohaline staircase. Deep-Sea Res., 34, 1689-1696.

Haldane, J. B. S., 1952: Simple tests for bimodality and bitangentiality. Ann. Eugen., 16, 359-364.

Hamilton, J. M., M. R. Lewis, and B. R. Ruddick, 1989: Vertical fluxes of nitrate associated with salt fingers in the world's oceans. J. Geophys. Res., 94, 2137-2145.

Hautala, S. L., and S. C. Riser, 1993: A nonconservative $\beta$-spiral determination of the deep circulation in the Eastern South Pacific. J. Phys. Oceanogr., 23, 1975-2000.

Henyey, F.S., J. Wright and S. M. Flatte, 1986: Energy and action flow though the internal wave field: an eikonal approach. J. Geophys. Res., 91, 8487-8495.

Hogg, N. G., P. Biscaye, E. Gardner and W. J. Schmitz, 1982: On the transport f Antarctic Bottom Water in the Vema Channel. J. Mar. Res., 40, Suppl., 231-263.

Hogg, N. G., and W. B. Owens, 1999: Direct measurements of the deep circulation within the Brazil basin. Deep-Sea Res. II, 46, 335-353.

Hug, P. and R. E. Britter, 1995: Turbulence evolution and mixing in a two layer stably stratified fluid. J. Fluid Mech., 285, 41-67.

Iselin, C. O. D., 1939: The influence of vertical and lateral turbulence on the characteristics of the waters at mid-depths. Trans. Amer. Geophys. Un., $20,414-417$. 
Itsweire, E. C., J. R. Koseff, D. A. Briggs and J. H. Ferziger, 1993: Turbulence in stratified shear flows: Implications for interpreting shear-induced mixing in the ocean. J. Phys. Oceanogr., 23, 1508-1522.

Jackett, D. R., and T. J. McDougall, 1997: A neutral density variable for the world's oceans. J. Phys. Oceanogr., 27, 237-263.

Joyce, T. M., 1980: On the production and dissipation of thermal variance in the oceans. J Phys. Oceanogr., 10, 460-463.

Joyce, T. M., B. A. Warren and L. D. Talley, 1986: The geothermal heating of the abyssal subarctic Pacific Ocean. Deep-Sea Res., 33, 1003-1015.

Joyce, M. T., J. R. Luyten, A. Kubryakov, F. B. Bahr, and J. S. Pallant, 1998: Meso- to large-scale structure of subducting water in the subtropical gyre of the eastern North Atlantic Ocean. J. Phys. Oceanogr., 28, 40-61.

Kunze, E., 1987: Limits on growing, finite-length salt fingers: A Richardson number constraint. J. Marine Res., 45, 533-556.

Kunze, E., 1990: The evolution of salt fingers in inertial wave shear. J. Marine Res., 48, 471-504.

Kunze, E., 1994: A proposed flux constraint for salt fingers in shear. J. Marine Res., 52, 999-1016.

Kunze, E., A. J. Williams, and R. W. Schmitt, 1987: Optical microstructure in the thermohaline staircase east of Barbados. Deep-Sea Res., 34, 1697-1704.

Kunze, E., and J. M. Toole, 1997: Tidally driven vorticity, diurnal shear, and turbulence atop Fieberling Seamount. J. Phys. Oceanogr., 27, 2663-2693.

Kunze, E., and T. B. Sanford, 1996: Abyssal mixing: Where it is not. J. Phys. Oceanogr., 26, 2286-2296.

Ledwell, J. R., A. J. Watson, and C. S. Law, 1993: Evidence for slow mixing across the pycnocline from an open-ocean tracer-release experiment. Nature, 364, 701-703. 
Ledwell, J. R., A. J. Watson, and C. S. Law, 1998: Mixing of a tracer released in the pycnocline of a subtropical gyre. J. Geophys. Res., 103, 21499-21529.

Ledwell, J. R., E. T. Montgomery, K. L. Polzin, L. C. St. Laurent, R. W. Schmitt and J. M. Toole, 1999: Mixing over rough topography in the Brazil Basin. Nature, submitted.

Linden, P. F., 1974: Salt fingers in a steady shear flow. Geophys. Fluid Dyn., 6, $1-27$.

Lueck, R., 1987: Microstructure measurements in a thermohaline staircase. DeepSea Res., 34, 1677-1688.

Lueck, R., and R. Reid, 1984: On the production and dissipation of mechanical energy in the ocean. J. Geophys., Res., 89, 3439-3445.

Marmorino, G. O., W. K. Brown and W. D. Morris, 1987: Two-dimensional temperature structure in the C-SALT thermohaline staircase. Deep-Sea Res., $34,1667-1675$.

Marotzke, J., 1997: Boundary mixing and the dynamics of three dimensional thermohaline circulations. J. Phys. Oceanogr., 27, 1713-1728.

McDougall, T. J., 1984: The relative roles of diapycnal and isopycnal mixing on subsurface water mass conversion. J. Phys. Oceanogr., 14, 1577-1589.

McDougall, T. J., 1988: Neutral-surface potential vorticity. Prog. Oceanog., 20, 185-221.

McDougall, T. J., 1991: Water mass analysis with three conservative variables. J Geophys. Res., 96, 8687-8693.

McDougall, T. J., and J. R. Taylor, 1984: Flux measurements across a finger interface at low values of the stability ratio. J. Marine Res., 42, 1-i4.

McDougall, T. J., and J. A. Whitehead, 1984: Estimates of the relative roles of diapycnal, isopycnal and double-diffusive mixing in Antarctic Bottom Water in the North Atlantic. J. Geophys. Res., 89, 10479-10483. 
McDougall, T. J., and B. R. Ruddick, 1992: The use of ocean microstructure to quantify both turbulent mixing and salt fingering. Deep-Sea Res., 39, 1931-1952.

Morris, M., N. Hogg and W. B. Owens, 1997: Diapycnal mixing estimated from advective budgets in the deep Brazil Basin. International WOCE Newsletter, 28, 23-35.

Moum, J. N., 1996: Efficiency of mixing in the main thermocline. J. Geophys. Res., 101, 12,057-12,069.

Moum, J. N., and T. R. Osborn, 1986: Mixing in the main thermocline. J. Phys. Oceanogr., 16, 1250-1259.

Munk, W. H., 1966: Abyssal recipes. Deep-Sea Res., 13, 207-230.

Munk, W., 1981: Internal waves and small-scale processes. Evolution of Physical Oceanography, B. A. Warren and C. Wunsch, Eds., The MIT Press, 264-291.

Munk, W., and C. Wunsch, 1998: Abyssal recipes II: energetics of tidal and wind mixing. Deep-Sea Res. I, 45, 1977-2010.

Oakey, N. S., 1985: Statistics of mixing parameters in the upper ocean during JASIN Phase 2. J. Phys. Oceanogr., 15, 1662-1675.

Olbers, D. J., M. Wenzel and J. Willebrand, 1985: The inference of North Atlantic circulation patterns from climatological hydrographic data. Rev. Geophys., $23,313-356$.

Osborn, T. R., 1980: Estimates of the local rate of vertical diffusion from dissipation measurements. J. Phys. Oceanogr., 10, 83-89.

Osborn, T. R., and C. S. Cox, 1972: Oceanic fine structure. Geophys. Fluid Dyn., 3, 321-345.

Pedlosky, J., 1996: Ocean Circulation Theory, Springer-Verlag, 453 pp.

Polzin, K. L., 1996: Statistics of the Richardson number: Mixing models and finestructure. J. Phys. Oceanogr., 26, 1409-1425. 
Polzin, K. L., 1999: Abyssal mixing: Inertial subrange solutions for the energy balance of the finescale internal wavefield. in preparation.

Polzin, K. L., J. M. Toole and R. W. Schmitt, 1995: Finescale parameterizations of turbulent dissipation. J. Phys. Oceanogr., 25, 306-328.

Polzin, K. L., and E. T. Montgomery, 1996: Proceedings of the ONR workshop on microstructure sensors, Mt. Hood, Oregon, 109-115.

Polzin, K. L., J. M. Toole, J. R. Ledwell and R. W. Schmitt, 1997: Spatial variability of turbulent mixing in the abyssal ocean. Science, 276, 93-96.

Robinson, A. R., and H. Stommel, 1959: The oceanic thermocline and the associated thermohaline circulation. Tellus, 11, 295-308.

Rohr, J. J., E. C. Itsweire, and C. W. Van Atta, 1984: Mixing efficiency in stably-stratified decaying turbulence. Geophys. Astrophys. Fluid Dyn., 29, 221-236.

Rohr, J. J., E. C. Itsweire, K. N. Helland and C. W. Van Atta, 1988: Growth and decay of turbulence in a stably stratified shear flow. J. Fluid Mech., 195, $77-111$.

Roemmich, D., S. Hautala and D. Rudnick, 1996: Northward abyssal transport through the Samoan Passage and adjacent regions. J. Geophys Res., 101, 14039-14055.

Ruddick, B., D. Walsh, and N. Oakey, 1997: Variations in apparent mixing efficiency in the North Atlantic Central Water. J. Phys. Oceanogr., 27, 25892605.

Rudnick, D. L., and R. Ferrari, 1999: Compensation of horizontal temperature and salinity in the ocean mixed layer. Science, 283, 526-529.

Schmitt, R. W., 1979a: The growth rate of super-critical salt fingers. Deep-Sea Res., 26, 23-40.\}

Schmitt, R. W., 1979b: Flux measurements on salt fingers at an interface. J. Marine Res., 37, 419-436. 
Schmitt, R. W., 1981: Form of the temperature-salinity relationship in the Central Water: Evidence for double-diffusive mixing. J. Phys. Oceanogr., 11, 1015-1026.

Schmitt, R. W., 1990: On the density ratio balance in Central Water. J. Phys. Oceanogr., 20, 900-906.

Schmitt, R.W., 1994: Double diffusion in oceanography. Annu. Rev. Fluid Mech., 26, 255-285.

Schmitt, R. W., 1999: Spice and the Demon. Science, 283, 498-499.

Schmitt, R. W., and D. L. Evans, 1978: An estimate of the vertical mixing due to salt fingers based on observations of North Atlantic Central Water. J. Geophys. Res., 83, 2913-2919.

Schmitt, R. W., H. Perkins, J. D. Boyd, and M. C. Stalcup, 1987: C-SALT: An investigation of the thermohaline staircase in the western tropical North Atlantic. Deep-Sea Res., 34, 1655-1665.

Schmitt, R. W., J. M. Toole, R. L. Koehler, E. C. Mellinger, and K. W. Doherty, 1988: The development of a fine- and microstructure profiler. J. Atmos. Ocean. Tech., 5, 484-500.

Shen, C.Y., 1993: Heat-salt finger fluxes across a density interface. Phys. Fluids A, 5, 2633-2643.

Shen, C.Y., 1995: Equilibrium salt-fingering convection. Phys. Fluids A, 7, 706717.

Smith, W. H. F., and D. T. Sandwell, 1997: Global sea floor topography from satellite altimetry and ship depth soundings. Science, 277, 1956-1962.

Spall, M. A., 1998: A simple model of the large scale circulation of Mediterranean Water and Labrador Sea Water. Deep-Sea Res. II, 46, 181-204.

Starr, V. P., 1968: Physics of Negative Viscosity Phenomena. McGraw-Hill, New York, 256pp. 
Stern, M. E., 1960: The "salt-fountain" and thermohaline convection. Tellus, 12, 172-175.

Stern, M. E., 1969: Collective instability of salt fingers. J. Fluid Mech., 35, 209218.

Stern, M. E., 1975: Ocean Circulation Physics, Academic Press, San Diego, CA, pp. 246.

Stern, M. E., and J. S. Turner, 1969: Salt fingers and convecting layers. Deep-Sea Res., 16, 497-511.

Stommel, H., 1957: The abyssal circulation of the ocean. Nature, 180. 733-734.

Stommel, H., 1958: The abyssal circulation. Letter to the Editors. Deep-Sea Res., 5, 80-82.

Stommel, H. M., 1993: A conjectural regulating mechanism for determining the thermohaline structure of the oceanic mixed layer. J. Phys. Oceanogr., 23, 142-148.

Stommel, H., A. B. Arons and A. J. Faller, 1959: Some examples of stationary planetary flow patterns in bounded basins. Tellus, 10, 1-11.

Stommel, H., and A. B. Arons, 1960: On the abyssal circulation of the World Ocean-I: Stationary planetary flow patterns on a sphere. Deep-Sea Res., 6. 140-154.

Stommel, H. and F. Schott, 1977: The beta spiral and the determination of the absolute velocity field from hydrographic station data. Deep-Sea Res., 24, $325-329$.

Saunders, P. M., 1987: Flow though Discovery Gap. J Phys. Oceanogr., 17, 631-643.

Swallow, J. C., 1971: The Aries Current measurements in the western North Atlantic.Philos. Trans. Roy. Soc. London A, 270, 451-460. 
Taylor, J., and P. Bucens, 1989: Laboratory experiments on the structure of salt fingers. Deep-Sea Res., 36, 1675-1704.

Tennekes, M., and J. L. Lumley, 1972: A First Course in Turbulence. Massachusetts Institute of Technology Press, Cambridge, MA, 300pp.

Thorpe, S. A., 1977: Turbulence and mixing in a Scottish Loch. Phil. Trans. Roy. Soc. London, A286, 125-181.

Toggweiler, J. R., and B. Samuels, 1995: Effect of Drake Passage on the global thermohaline circulation. Deep-Sea Res., 42, 477-500.

Toggweiler, J. R., and B. Samuels, 1998: On the ocean's large scale circulation near the limit of no vertical mixing. J. Phys. Oceanogr., 28, 1832-1852.

Toole, J. M., K. L. Polzin, and R. W. Schmitt, 1994: Estimates of diapycnal mixing in the abyssal ocean. Science, 264, 1120-1123.

Toole, J. M., R. W. Schmitt, K. L. Polzin, and E. Kunze, 1997a: Near-boundary mixing above the flanks of a midlatitude seamount. J. Geophys. Res., 102, 947-959.

Toole, J. M., J. R. Ledwell, K. L. Polzin, R. W. Schmitt, E. T. Montgomery, L. St. Laurent and W. B. Owens, 1997b: The Brazil basin Tracer Release Experiment. International WOCE Newsletter, 28, 25-28.

Trowbridge, J., and O. S. Madsen, 1984: Turbulent wave boundary layers 1: Model formulation and first-order solution. J. Geophys. Res., 89, 79897997.

Turner, J. S., 1967: Salt fingers across a density interface. Deep-Sea Res., 14, $599-611$.

Tziperman, E., and A. Hecht, 1988: Circulation in the Eastern Levantine Basin determined by inverse methods. J. Phys. Oceanogr., 18, 506-518.

Veronis, G., 1975: The role of models in tracer studies. Numerical Models of the Ocean Circulation, National Academy of Science, 133-146. 
Whitehead, J. A., and L. V. Worthington, 1982: The flux and mixing rates of Antarctic Bottom Water within the North Atlantic. J. Geophys. Res., 87, 7903-7924.

Wijffels, S. E., 1993: Exchanges between gyres and hemispheres: A direct approach to the mean circulation of the equatorial Pacific Ocean. Ph. D. dissertation, MIT-WHOI Joint Program in Oceanography, 294 pp.

Winters, K. B., and E. A. D'Asaro, 1996: Diascalar flux and the rate of fluid mixing. J. Fluid Mech., 317, 179-193.

Wunsch, C., 1994: Dynamically consistent hydrography and absolute velocity in the eastern North Atlantic Ocean. J. Geophys. Res., 99, 14071-14090.

Wunsch, C., 1996: The Ocean Circulation Inverse Problem. Cambridge University Press, $442 \mathrm{pp}$.

Yamazaki, H., and T. R. Osborn, 1990: Dissipation estimates for stratified turbulence. J. Geophys. Res., 95, 9739-9744.

Zhang, H.-M., and N. G. Hogg, 1992: Circulation and water mass balance in the Brazil Basin. J. Marine Res., 50, 385-420.

Zhang, J., R. W. Schmitt, and R. X. Huang, 1998: Sensitivity of the GFDL Modular Ocean Model to the parameterization of double-diffusive processes. J. Phys. Oceanogr., 28, 589-605.

Zhang, J., R. W. Schmitt, and R. X. Huang, 1999: The relative influence of diapycnal mixing and hydrologic forcing on the stability of the thermohaline circulation. J. Phys. Oceanogr., 29, 1096-1108. 